Accepted to ApJS 2015 June 27

\title{
The Brown Dwarf Kinematics Project (BDKP). IV. Radial Velocities of 85 Late-M and $L$ dwarfs with MagE
}

\author{
Adam J. Burgasser ${ }^{1}$, Sarah E. Logsdon ${ }^{2}$, Jonathan Gagné ${ }^{3}$, John J. Bochanski ${ }^{4}$, Jaqueline K. \\ Faherty $^{5,6,7}$, Andrew A. West ${ }^{8}$, Eric E. Mamajek ${ }^{9}$, Sarah J. Schmidt ${ }^{10}$, and Kelle L. Cruz ${ }^{6,11,12}$
}

\begin{abstract}
Radial velocity measurements are presented for 85 late M- and L-type very low mass stars and brown dwarfs obtained with the Magellan Echellette (MagE) spectrograph. Targets primarily have distances within 20 pc of the Sun, with more distant sources selected for their unusual spectral energy distributions. We achieved precisions of $2-3, \mathrm{~km} \mathrm{~s}^{-1}$, and combined these with astrometric and spectrophotometric data to calculate $U V W$ velocities. Most are members of the thin disk of the Galaxy, and velocity dispersions indicate a mean age of $5.2 \pm 0.2$ Gyr for sources within 20 pc. We find signficantly different kinematic ages between late-M dwarfs (4.0 $\pm 0.2 \mathrm{Gyr})$ and $\mathrm{L}$ dwarfs $(6.5 \pm 0.4$ Gyr) in our sample that are contrary to predictions from prior simulations. This difference appears to be driven by a dispersed population of unusually
\end{abstract}

\footnotetext{
${ }^{1}$ Center for Astrophysics and Space Science, University of California San Diego, La Jolla, CA, 92093, USA; aburgasser@ucsd.edu

${ }^{2}$ Department of Physics and Astronomy, UCLA, 430 Portola Plaza, Box 951547, Los Angeles, CA 90095-1547, USA

${ }^{3}$ Institute for Research on Exoplanets (iREx), Université de Montréal, Département de Physique, C.P. 6128 Succ. Centre-ville, Montréal, QC H3C 3J7, Canada

${ }^{4}$ Rider University, 2083 Lawrenceville Road, Lawrenceville, NJ 08648, USA

${ }^{5}$ Department of Terrestrial Magnetism, Carnegie Institution of Washington, Washington, DC 20015, USA

${ }^{6}$ Department of Astrophysics, American Museum of Natural History, Central Park West at 79th Street, New York, NY 10034, USA

${ }^{7}$ Hubble Postdoctoral Fellow

${ }^{8}$ Department of Astronomy, Boston University, 725 Commonwealth Ave Boston, MA 02215 USA

${ }^{9}$ Department of Physics \& Astronomy, University of Rochester, Rochester, NY 14627, USA

${ }^{10}$ Department of Astronomy, Ohio State University, 140 West 18th Avenue, Columbus, OH 43210, USA

${ }^{11}$ Department of Physics and Astronomy, Hunter College, 695 Park Avenue, New York, NY10065, USA

${ }^{12}$ Department of Physics, Graduate Center, City University of New York, 365 Fifth Avenue, New York, NY 10016, USA
} 
blue L dwarfs which may be more prevalent in our local volume-limited sample than in deeper magnitude-limited surveys. The $\mathrm{L}$ dwarfs exhibit an asymmetric $U$ velocity distribution with a net inward flow, similar to gradients recently detected in local stellar samples. Simulations incorporating brown dwarf evolution and Galactic orbital dynamics are unable to reproduce the velocity asymmetry, suggesting non-axisymmetric perturbations or two distinct $\mathrm{L}$ dwarf populations. We also find the $\mathrm{L}$ dwarfs to have a kinematic age-activity correlation similar to more massive stars. We identify several sources with low surface gravities, and two new substellar candidate members of nearby young moving groups: the astrometric binary DENIS J08230313-4912012AB, a lowprobability member of the $\beta$ Pictoris Moving Group; and 2MASS J15104786-2818174, a moderate-probability member of the 30-50 Myr Argus Association.

Subject headings: stars: low-mass, brown dwarfs; stars: kinematics and dynamics; methods: statistical; techniques: radial velocities

\section{Introduction}

Very low-mass (VLM) stars and brown dwarfs with masses $\lesssim 0.1 \mathrm{M}_{\odot}$ comprise a significant fraction of stars in our Galaxy ( $\gtrsim 20 \%$; Chabrier 2003; Bochanski et al. 2010). Their ubiquity and extremely long lifetimes make them an important probe of Galaxy structure, chemical evolution and star formation history (Burgasser 2004; Bochanski et al. 2007a; Pirzkal et al. 2009; Pineda et al. 2013). However, the low luminosities and temperatures of these M, L, T and Y dwarfs (Kirkpatrick 2005), and the steady cooling of substellar VLM dwarfs over time, has made it difficult to identify and study them in statistically significant numbers. Fortunately, the completion of wide-field red-optical and infrared sky surveys such as the DEep Near-Infrared Survey of the Southern Sky (DENIS; Epchtein et al. 1997), the Two Micron All Sky Survey (2MASS; Skrutskie et al. 2006), the Sloan Digital Sky Survey (SDSS; York et al. 2000), the Canada-France Hawaii Telescope Legacy Survey (CFHTLS; Delorme et al. 2008), and United Kingdom Infrared Telescope Deep Sky Survey (UKIDSS; Lawrence et al. 2007); and the ongoing Wide-field Infrared Survey Explorer (WISE; Wright et al. 2010), Panoramic Survey Telescope and Rapid Response System (Pan-STARRS; Kaiser et al. 2002), and Visible and Infrared Survey Telescope for Astronomy (VISTA; Emerson et al. 2004) have uncovered roughly 10,000 VLM dwarfs within 100 pc of the Sun 1 . The detector technologies enabling these surveys have also led to advances in spectroscopic instrumentation, allowing detailed characterization of spectral energy distributions and corresponding physical parameters for increasingly larger samples of VLM dwarfs (e.g., Reid et al. 2008; Schmidt et al. 2010; West et al. 2011; Kirkpatrick et al. 2012).

\footnotetext{
${ }^{1}$ Current compilations are maintained by C. Gelino at http://dwarfarchives.org and J. Gagne at https://jgagneastro.wordpress.com/list-of-ultracool-dwarfs
} 
Characterizing VLM dwarfs as a population relies on accurate measures of their individual characteristics, including kinematics. While most stars in the disk of the Milky Way form in the mid-plane, with Galactic orbits similar to that of the Sun (low inclination, low eccentricity), dynamical encounters with giant molecular clouds, spiral structure and other gravitational potential gradients scatter stars stochastically (Spitzer \& Schwarzschild 1953; Toomre 1964; Junqueira et al. 2013). Individual orbits may be chaotic, but the population as a whole evolves toward greater velocity dispersion over time (Wielen 1977). The age- velocity dispersion relation has been used extensively in studies of Galactic star formation history with nearby main sequence stars (e.g., Dehnen \& Binney 1998; Binney et al. 2000; Aumer \& Binney 2009). Stellar kinematics also segregate large-scale Galactic populations - the thin disk, thick disk and halo-which trace Galactic structure, formation history and chemical enrichment (e.g., Gilmore \& Reid 1983; Reid \& Majewski 1993; Carollo et al. 2008). Young moving groups (YMGs) near the Sun can also be revealed by coherently moving stars with common spectral signatures of low surface gravity or abundance patterns (e.g., Zuckerman \& Song 2004; López-Santiago et al. 2006; Torres et al. 2006; Gagné et al. 2014). Finally, periodicity in motion identifies low-mass companions that can be used to make direct mass measurements and test substellar/exoplanet evolutionary models (Martin et al. 1999; Dupuy et al. 2009a; Konopacky et al. 2010; Sahlmann et al. 2013).

Full characterization of the three-dimensional motions of VLM dwarfs requires radial velocities (RVs), and hence high resolution spectroscopy, observations which have proven challenging for these faint sources. Only a small fraction of the known VLM population has sufficiently precise $\left(<5 \mathrm{~km} \mathrm{~s}^{-1}\right)$ RV measurements necessary for robust membership assignment or RV variability detection (e.g., Tinnev \& Reid 1998; Reid et al. 2002b; Basri \& Reiners 2006; Blake et al. 2007, 2010; Zapatero Osorio et al. 2007; Reiners \& Basri| 2009; Seifahrt et al. 2010; Tanner et al. 2012). Nevertheless, these studies have identified several remarkable - and in some cases conflicting - kinematic trends among the population. Zapatero Osorio et al. (2007) examined the velocity dispersions for 31 late- $\mathrm{M}$ and $21 \mathrm{~L}$ and $\mathrm{T}$ dwarfs, finding the latter to be less dispersed and hence marginally younger, $1.2_{-0.7}^{+1.1} \mathrm{Gyr}$ versus $3.8_{-1.9}^{+2.8} \mathrm{Gyr}$ based on the age-dispersion relation of Wielen (1977). This age difference is qualitatively consistent with population synthesis simulations that predict that L dwarfs should be on average younger due to brown dwarf cooling (Burgasser 2004; Allen et al. 2005). However, a large fraction $(\sim 40 \%)$ of the $\mathrm{L}$ and $\mathrm{T}$ dwarfs examined in that study were also identified as kinematic members of the 0.4-2 Gyr Hyades Stream, which may have biased their collective ages downward. More recent work by Reiners \& Basri (2009); Seifahrt et al. (2010) and Blake et al. (2010), based on precise RV measurements for roughly $150 \mathrm{M}$ and L dwarfs and more accurate application of the Wielen relations, find mean ages of 3 Gyr and 5 Gyr for late-M and L dwarfs, respectively; i.e., the reverse trend. Tangential velocity studies by Faherty et al. (2009) and Kirkpatrick et al. (2010), and a larger but lower-precision RV sample by Schmidt et al. (2010), find equivalent ages for M, L and T dwarfs, of order 3-8 Gyr, with no statistically significant difference.

Faherty et al. (2009) and Schmidt et al. (2010) also report color-dependent trends in VLM dwarf kinematics, with sources identified as unusually blue in near-infrared colors being more 
widely dispersed than those with unusually red colors. These differences were attributed to age and/or metallicity effects, in particular increased collision-induced $\mathrm{H}_{2}$ absorption in the atmospheres of sources with high surface gravities and/or subsolar metallicities (cf. Burgasser et al. 2008; Jameson et al. 2008; Looper et al. 2008). However, Kirkpatrick et al. (2010) found no difference in dispersions between red and blue outliers among a proper-motion selected sample of $\mathrm{L}$ dwarfs, with both sets appearing to be drawn from an older population.

To address these disagreements in the velocity dispersions and age determinations for the local VLM dwarf population, we report RV measurements for 85 late-type M and L dwarfs based on data obtained with the Magellan Echellete spectrograph (MagE; Marshall et al. 2008). These include measurements for 30 dwarfs without previously reported RVs. In Section 2 we describe our sample, observations, and data reduction methods. In Section 3 we discuss our RV measurement procedures in detail, comparing the accuracy and precision of three common methods. In Section 4 we merge our RV measurements with proper motion and distance determinations to calculate UVW space velocities, and assign membership to Galactic thin and thick disk populations. We also examine velocity dispersions, probability distributions and kinematic ages for the full sample and subsamples based on spectral class, color and activity. In Section 5 we focus on the distinct velocity distributions of $\mathrm{M}$ and $\mathrm{L}$ dwarfs in our sample, and use population synthesis plus orbit simulations in an attempt to reproduce the asymmetries in the $U$ velocity distribution of the latter. In Section 6 we consider kinematic and spectroscopic evidence for association in nearby YMGs, and report two new brown dwarf candidate members. In Section 7 we highlight additional individual sources of interest in detail. Results are summarized in Section 8.

\section{Observations}

\subsection{Sample}

Our observational sample is summarized in Table 1. We initially selected 85 late-type dwarfs with published optical spectral types M7 through L5, primarily from the Palomar/MSU Survey (Reid et al. 1995; Hawlev et al. 1996; Reid et al. 2002a); the 2MASS surveys of Kirkpatrick et al. (1999, 2000); Gizis (2002); Cruz et al. (2003, 2007) and Reid et al. (2008); the SDSS surveys of Bochanski et al. (2005); West et al. (2008, 2011) and Schmidt et al. (2010); the DENIS surveys of Delfosse et al. (1999); Martín et al. (1999); Kendall et al. (2004, 2007) and Phan-Bao et al. (2008); and other individual discoveries. The majority of our sample were selected to have declinations $\delta \leq 25^{\circ}$ (Figure 11) and distances $d \lesssim 20 \mathrm{pc}$, the latter based on either astrometric parallax measurements (Tinney et al. 1995; van Altena et al. 1995; Dahn et al. 2002; Costa et al. 2005, 2006; Henry et al. 2006; Andrei et al. 2011; Faherty et al. 2012; Dieterich et al. 2014) or spectrophotometric distance estimates based on 2MASS $J$-band photometry and the $M_{J} /$ spectral type relation

of Cruz et al. (2003). Sources within 20 pc $(1 \sigma)$ comprise $82 \%$ of our sample. Fifteen sources are at larger distances, most due to previously underestimated distances (e.g., due to multiplicity, misclas- 
sification), but several were specifically targetted due to their unusual spectral features indicative of surface gravity, metallicity or cloud effects. There were also several well-resolved binaries selected (e.g., J2200-3038AB2 ) and sources with unusually large tangential velocities (e.g., J0923+2340). We emphasize that while our sample was primarily designed to be volume-limited, it is not volumecomplete. Space density estimates from Cruz et al. (2007) predict $\sim 160$ late-M dwarfs and $\sim 125$ L dwarfs within $20 \mathrm{pc}$, so the 70 sources in our sample satisfying this distance limit represents $\sim 25 \%$ of the total local population. Figure 2 displays a breakdown of our sample by spectral type (based on updated classifications; see below); $56 \%$ are late-type M dwarfs, $44 \%$ are L dwarfs. We also display the 2MASS $J-K_{s}$ colors of our targets as compared to the median color relations of Schmidt et al. (2010) and West et al. (2011) based on SDSS sources. There appears to be a systematic offset in the $J-K_{s}$ colors of our sample relative to these surveys, with our M dwarfs being on average redder and our $\mathrm{L}$ dwarfs being on average bluer. The red offset in our $\mathrm{M}$ dwarfs is likely due to our more local sample, as the M dwarfs from West et al. (2011) extend to >100 pc above/below the Galactic disk, and are likely to be on average more metal-poor. The blue color offset for our L dwarfs, however, is unclear. We compiled published proper motions and tangential velocities, or in the absence of a measurement compared multi-epoch astrometry between 2MASS and WISE, yielding typical precisions of 5-15 mas $\mathrm{yr}^{-1}$; see Gagné et al. (2015) for details.

As of March 2015, 43 sources in our sample had RV measurements reported in the literature with precisions $\sigma_{R V} \leq 3 \mathrm{~km} \mathrm{~s}^{-1}$ (Tinnev \& Reid 1998; Mohanty \& Basri 2003; Reiners \& Basri 2009; Blake et al. 2010; Gálvez-Ortiz et al. 2010; Seifahrt et al. 2010; Tanner et al. 2012); these values are listed in Table 1. We made use of these prior measurements in our RV measurement analysis (Section 3.3).

\subsection{MagE Observations}

All sources were observed with the MagE spectrograph, mounted on the Magellan 6.5m Landon Clay Telescope at Las Campanas Observatory. A complete observing log is given in Table 2, Data were obtained in 15 nights over a 2.5-year period (November 2008 through March 2011) in a variety of seeing and weather conditions. We used the 0.7 slit aligned with the parallactic angle, providing 3200-10050 $\AA$ spectroscopy at an average resolution $\lambda / \Delta \lambda \approx 4100\left(\Delta \mathrm{RV}=73 \mathrm{~km} \mathrm{~s}^{-1}\right)$ and dispersion of $\sim 0.5 \AA$ pixel $^{-1}$ at $6000 \AA$. Exposure times varied according to source brightness and weather conditions, and ranged from 150-3600 s. Most sources were observed in a single exposure, although a handful were observed in multiple exposures or over multiple nights to improve data quality. In addition to the target, we obtained nightly observations of spectrophotometric standards from Hamuy et al. (1994) for flux calibration. ThAr lamps were observed after each source observation for wavelength calibration, and internal quartz and dome flat field lamps were obtained

\footnotetext{
${ }^{2}$ Source identifications in the text are given in shorthand notation based on the sexigesimal right ascension and declination, Jhhmm \pm ddmm. Full source names and coordinates are listed in Table 1
} 
on each night for pixel response calibration. Data were reduced using the MASE reduction pipeline (Bochanski et al. 2009), following standard procedures for order tracing, flat field correction, wavelength calibration (including correction to heliocentric motion), optimal source extraction, order stitching, and flux calibration. We did not perform any correction for telluric absorption on these data, which caused a problem for the flux calibration for several of the spectra around the 7500$7600 \AA_{2} \mathrm{O}_{2}$ telluric band, which resides near an echelle order boundary. This region is ignored in subequent analyses. We also note that the spectrum of J0148-3024 was affected by poor flux calibration in the 8300-8700 $\AA$ band, and the region is also ignored in the analysis of this source.

\subsection{Spectral Features and Classification}

The red optical components of our spectra (6000-9000 $\AA$ ) are shown in Figure 3 . The data are generally of high quality, with signal-to-noise ratios $(\mathrm{S} / \mathrm{N})$ of $20-200$ at $8600 \AA$ (mean $\mathrm{S} / \mathrm{N}=60$ ). We see all of the characteristic features of late-type dwarfs, including strong $\mathrm{TiO}, \mathrm{VO}, \mathrm{CaH}$ and metal line absorption in the late-M dwarfs. The features fade in the $\mathrm{L}$ dwarf spectra and $\mathrm{FeH}, \mathrm{CrH}$, and various alkali lines emerge, including the heavily pressure-broadened $\mathrm{Na}$ I and $\mathrm{K}$ I doublets centered at $5500 \AA$ and $7700 \AA$, respectively (Kirkpatrick et al. 1991, 1999; Martín et al. 1999).

We revisited the optical classifications for all of our sources by comparing directly to the SDSS $\mathrm{M}$ and L dwarf templates from Bochanski et al. (2007b) and Schmidt et al. (2014) over the 7100$8800 \AA$ range (excluding the $7500-7600 \AA \mathrm{O}_{2}$ band). The best-match template $(T(\lambda))$ was identified as that with the minimum squared deviation from the observed spectrum $(f(\lambda))$ :

$$
\sigma^{2}=\sum_{\lambda=7100 \AA}^{8800 \AA}(f(\lambda)-\alpha T(\lambda))^{2}
$$

where

$$
\alpha=\frac{\sum_{\lambda=7100 \AA}^{8800 \AA} f(\lambda) T(\lambda)}{\sum_{\lambda=7100 \AA}^{8800 \AA} \mathrm{\circ} T(\lambda)^{2}}
$$

is the optimal scale factor. For most of our sample, these classifications are within 1 subtype of those reported in the literature. The six discrepant sources (J0041-5621AB, J0123-6921, J0331-3042, J0641-4322, J0751-2530, J0823-4912AB) were re-examined by eye and the revised classification

confirmed. Note that three of these discrepant sources exhibit signatures of low surface gravity, as discussed below.

\subsection{Emission and Absorption Line Features}

For the majority of our sources, we also detect Balmer line emission, including $\mathrm{H} \alpha, \mathrm{H} \beta$ and $\mathrm{H} \gamma$ lines. Figure 4 displays the fraction of sources with detectable $\mathrm{H} \alpha$ emission as a function of spectral 
type. Similar to previous studies (e.g., Gizis et al. 2000; Kirkpatrick et al. 2000; West et al. 2004, 2008; Schmidt et al. 2007, 2014) we find a decline in the frequency of emission between the late-M dwarfs (96\% active) and the L dwarfs (54\% active), although a trend with spectral type is difficult to quantify given the relatively small sample.

We computed the relative $\mathrm{H} \alpha$ luminosities $\left(\log _{10} L_{H \alpha} / L_{b o l}\right)$ by two methods. First, we used the sources' 2MASS $J$ magnitudes and mean $i-J$ colors as a function of spectral type from Schmidt et al. (2010) and West et al. (2011) to scale the spectra and convert H $\alpha$ emission into apparent flux units. We then used $J$-band bolometric corrections from Liu et al. (2010) to compute apparent bolometric fluxes. The bolometric corrections are based on the Mauna Kea Observatory (MKO) filter system (Simons \& Tokunaga 2002; Tokunaga et al. 2002), so we computed a spectral type-dependent correction between 2MASS $J$ and MKO $J$ using spectrophotometry from 533 optically-classified M6-L7 dwarfs in the SpeX Prism Library (Burgasser 2014), as described in the Appendix. The ratio of apparent $\mathrm{H} \alpha$ flux to apparent bolometric flux yields $\log _{10} L_{H \alpha} / L_{b o l}$. As a second approach, we used the spectral type-dependent $\chi$-factor defined by Walkowicz et al. (2004), with updated values from Douglas et al. (2014) and Schmidt et al. (2014). Both methods gave consistent results, and values from the first method are listed in Table 3. As in previous studies, we find that the strength of emission monotonically declines from M7 to L5, ultimately falling below our detection limits. However, two L dwarfs, the L3 J2036+1051 and the L5 J1315-2649AB, stand out as being unusually active. The latter is a "hyperactive" L dwarf observed to have strong $\mathrm{H} \alpha$, alkali line and radio emission (Hall 2002b) a; Gizis 2002; Kirkpatrick et al. 2008; Burgasser et al. 2011, 2013). We also identify J2037-1137 as the only M8 dwarf in our sample to show no sign of $\mathrm{H} \alpha$ emission to a stringent limit $\left(\log _{10} L_{H \alpha} / L_{b o l}<-6.7\right)$. Both J2036+1051 and J2037-1137 are discussed further in Section 7.

Table 3 also lists equivalent widths (EWs) for alkali lines observed in the MagE data. Of particular note is Li I absorption detected in the spectra of nine sources, all shown in detail in Figure 5. We confirm the detection of Li I in J0041-5621AB, J0123-6921, J0823-4912, J1139-1159, J1411-2119 and J2045-6332 (Tinnev 1998; Mohanty et al. 2003; Reiners \& Basri 2009; Gálvez-Ortiz et al. 2014; Sahlmann et al. 2015), and find marginal evidence of absorption previously reported in J0339-3525 (Reiners \& Basri 2009). We report the first identification of Li I in the spectra of the M9 dwarfs J0652-2534 and J1510-2818. This line is a key age and mass indicator, as it is only present in cool dwarfs less massive than $0.06 \mathrm{M}_{\odot}$ and/or younger than 200 Myr (Magazzu et al. 1993; Bildsten et al. 1997; Burke et al. 2004). The presence of Li I absorption in the spectra of these dwarfs therefore indicates that they are likely to be young brown dwarfs.

\subsection{Low Surface Gravity Indicators}

As described in previous studies (e.g., Martín et al. 1999; Kirkpatrick et al. 2008; Cruz et al. 2009; Allers \& Liu 2013), young brown dwarfs with low surface gravities and low photospheric 
pressures exhibit enhanced $\mathrm{TiO}$ and $\mathrm{VO}$ absorption, weak metal hydride bands, and weak alkali lines compared to their equivalently-classified field dwarf counterparts. Building on work by Cruz et al. (2009), we quantified these features using the Na-a, Na-b, TiO-b, CrH-a, and FeH-a indices defined in Kirkpatrick et al. (1999), which sample the $8183 \AA$ and $8194 \AA$ Na I doublets, $8400 \AA$ TiO band, and $8580 \AA \mathrm{CrH}$ and $8660 \AA \mathrm{FeH}$ bands. We defined a new index (VO7900) sampling the $7900 \AA \mathrm{VO}$ band as the ratio of integrated flux between 7950-8000 $\AA$ over that between $7825-7875 \AA$. Table 4 lists the mean and standard deviations of these index combinations as a function of spectral type for sources without Li I absorption.

We identified sources in our sample for which alkali or metal-hydride bands are consistently weaker, and VO or TiO bands consistently stronger, than these spectral type means. These sources are listed in Table 5. Five show clear signatures of low surface gravity as compared to SDSS templates (Figure 6). Three of these sources exhibit Li I absorption and three have been identified as candidate kinematic members of the YMGs TW Hydrae (J1139-3159; Gizis 2002) and $\beta$ Pictoris (J2000-7523, J2045-6332; Gálvez-Ortiz et al. 2010; Gagné et al. 2014). An additional six sources (Figure 17) exhibit weak signatures of low surface gravity. All of these have Li I absorption, and three are previously noted as candidate kinematic members of the Tucana Horologium, Castor and Argus YMGs (Ribas 2003; Gálvez-Ortiz et al. 2010; Gagné et al. 2014). The low-gravity sources are discussed in further detail below.

\subsection{Metallicity Indicators}

For all of our late-M dwarfs, we computed the metallicity index $\zeta$ defined in Lépine et al. (2007). Within our sample, there is very little variation in $\zeta$, with all but one source (the M9.5 J0024-0158; $\zeta=0.56 \pm 0.03)$ falling into the "subdwarf" classification. Even this source is a poor metal-poor candidate given its late spectral type, although it is unusual in its combination of rapid rotation and sporadic (and occasionally flaring) magnetic emission (Basri \& Marcv 1995; Reid et al. 1999; Berger 2002; Berger et al. 2010). No H $\alpha$ emission from this source was detected in our data.

\section{Radial Velocity Measurements}

To obtain the most precise radial velocity measurements for $U V W$ space motion analysis, we examined three different measurement methods. For interested readers, we detail these methods here; final results are summarized in Table 6 . 


\subsection{Line Center Measurements}

Our first approach was to measure Doppler shifts for the prominent absorption and emission lines present in the data: K I, Rb I, Na I, Cs I, and $\mathrm{H} \alpha$. Line centers were determined by fitting Gaussian profiles to each line using the IRAF 3 routine splot, and these were compared to vacuum wavelengths obtained from the National Institute of Standards and Technology (NIST) Atomic Spectra Line database 4 . After rejecting poor line fits, we computed the average and standard deviation of the corresponding velocity shifts (Table 66).

Figure 8 compares our measured line center RVs to literature values for those sources with previous high precision measurements $\left(\sigma_{R V} \leq 3 \mathrm{~km} \mathrm{~s}^{-1}\right)$. Overall we find good agreement between these measurements, with a mean offset of $1.0 \pm 4.2 \mathrm{~km} \mathrm{~s}^{-1}$ (line center measurements are slightly more positive). In only one case, J0517-3349, do we find a significant discrepancy between our measurement $\left(-36 \pm 7 \mathrm{~km} \mathrm{~s}^{-1}\right)$ and that reported by Reiners \& Basri (2009, $\left.31.4 \mathrm{~km} \mathrm{~s}^{-1}\right)$, which we attribute to a sign reversal in that study. The median measurement uncertainties from this analysis are $7 \mathrm{~km} \mathrm{~s}^{-1}$, roughly one-tenth our nominal resolution but still unacceptably large for kinematic analysis.

\subsection{Cross-correlation with SDSS Templates}

To make better use of the full spectrum, we performed a cross-correlation analysis comparing to two sets of RV standards: the zero-velocity, low-resolution M7-L0 SDSS spectral templates of Bochanski et al. (2007b) and MagE observations of VLM dwarfs with published RV measurements. In both cases, we used the IDI 5 xorl package (Mohanty \& Basri 2003; West \& Basri 2009) to cross-correlate spectral pairs over five bands sampling distinct spectral features: $\mathrm{H} \alpha$ emission (6500$6600 \AA)$; the $7050 \AA$ TIO absorption band (7150-7250 $)$; K I and Rb I doublets (7685-7885 $\AA$ ); the

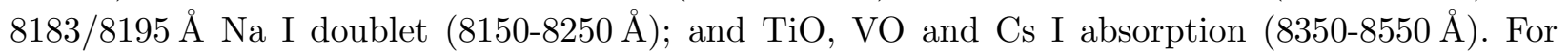
each source/template pair and spectral region, cross-correlation functions were visually inspected and poor correlations rejected.

For the SDSS template sample, we used the non-active templates to compare against our M7L0 sources. As the templates have a lower resolution $(\lambda / \Delta \lambda \approx 1800)$ than our MagE data, we smoothed the MagE data to an equivalent resolution using a Gaussian kernel and interpolated onto a common wavelength scale. Each source was compared to the template with the equivalent type;

\footnotetext{
${ }^{3}$ Image Reduction and Analysis Facility (Tody 1986). IRAF is distributed by the National Optical Astronomy Observatory, which is operated by the Association of Universities for Research in Astronomy, Inc., under cooperative agreement with the National Science Foundation

${ }^{4}$ http://physics.nist.gov/PhysRefData/ASD/lines_form.html

${ }^{5}$ Interactive Data Language
} 
for sources with half-subtype classifications, we compared to both the lower and higher integer subtype template. Velocity shifts from each band (and multiple templates) were then averaged and the standard deviation used as an estimate of the uncertainty. The latter were typically $\sim 5 \mathrm{~km} \mathrm{~s}^{-1}$, slightly smaller than our line center uncertainties.

Figure 8 compares our RVs based on this method to literature values. Unlike the line center measurements, we find a marginally significant offset in our measurements of $6.5 \pm 3.7 \mathrm{~km} \mathrm{~s}^{-1}$ (excluding J0517-3349), with the SDSS measurements being consistently more positive. To assess whether this was a consequence of our smoothing function, we performed the same cross-correlation on unsmoothed data and found a comparable offset. As the SDSS templates are shifted to a zero velocity rest frame in vacuum wavelengths using several of some of the same lines employed in our line centering analysis (K I, Na I and $\mathrm{H} \alpha$ ), we are unable to identify the origin of this offset.

\subsection{Cross-correlation with MagE Radial Velocity Standards}

Our third approach was to use the spectra of 40 sources in our sample with independently measured, high-precision RVs as cross-correlation standards. We matched sources to standards that had equivalent spectral types to within \pm 1 subtype (excluding the source itself if it was a standard), shifted the standards to zero velocity, and cross-correlated in the five spectral regions listed above. This resulted in up to 50 measurements per source, more for the late-M dwarfs. Following the same rejection and averaging procedures above, our reported values are listed in Table 6. Typical uncertainties are $\sim 1.3 \mathrm{~km} \mathrm{~s}^{-1}$, with over half of the sources having uncertainties below $1 \mathrm{~km} \mathrm{~s}^{-1}$; however, mid-type L dwarfs like J1750-0016 have higher uncertainties due to the fewer RV standards available with equivalent spectral types.

Figure 8 compares these measurements to the literature values, and we find that they are overall consistent, deviating on average by $0.2 \pm 2.7 \mathrm{~km} \mathrm{~s}^{-1}$ (again excluding J0517-3349). However the $\chi^{2}$ deviation is somewhat high in this case $\left(\chi^{2}=123, N=55, p=4 \times 10^{-7}\right)$, suggesting that our uncertainties are underestimated. We therefore include a $2 \mathrm{~km} \mathrm{~s}^{-1}$ systematic uncertainty in our reported values, added in quadrature with the standard deviations, which lowers the $\chi^{2}$ to a value consistent with no deviation $(p=0.1)$. The MagE cross-correlation measurements are also in agreement with the line center measurements (average deviation $-0.8 \pm 3.2 \mathrm{~km} \mathrm{~s}^{-1}$ ) but differ from the SDSS measurements $\left(-6.6 \pm 4.5 \mathrm{~km} \mathrm{~s}^{-1}\right)$. Given the smaller uncertainties and overall fidelity with prior measurements, we adopt the MagE standard cross correlations with an additional systematic uncertainty as our final RV measurements. The only exception is J1750-0016, whose large uncertainty $\left(14 \mathrm{~km} \mathrm{~s}^{-1}\right)$ was due to lower $\mathrm{S} / \mathrm{N}$ data $(\sim 23$ at $7400 \AA)$ and the availability of only one RV standard; for this source alone we adopt the line center value (Table 6). 


\subsection{Discrepant Radial Velocities}

In addition to J0517-3349, there are three sources whose measured velocities differ by about $3 \sigma$ or more from previously published values. The $\mathrm{L}$ dwarfs $\mathrm{J} 0500+0330$ and J0835+0819 have radial velocities from Blake et al. (2010) that are more than $10 \mathrm{~km} \mathrm{~s}^{-1}$ different from our crosscorrelation measures, our values being higher and lower, respectively. For these sources, the lack of mid-L dwarf MagE templates, and noise in some spectral regions used for cross-correlation, resulted in only 3-5 cross-correlation measurements. Hence, we conclude that our measurement uncertainties are likely underestimated. For the M8 J2351-2537, our value $\left(-12 \pm 3 \mathrm{~km} \mathrm{~s}^{-1}\right)$ deviates by $2.8 \sigma$ from that of Seifahrt et al. (2010, $\left.-3.0 \pm 1.1 \mathrm{~km} \mathrm{~s}^{-1}\right)$, but is in agreement with that of Reiners \& Basri (2009, $\left.-10 \pm 3 \mathrm{~km} \mathrm{~s}^{-1}\right)$. Hence, either the Seifahrt measure is in error or this source is binary RV variable. Additional observations are warranted to test the latter hypothesis.

\section{Analysis}

\section{1. $\quad U V W$ Space Motions and Kinematic Populations}

We combined our RV measurements with the proper motions and distances listed in Table 1 to compute heliocentric $U V W^{6}$ space velocities in the Local Standard of Rest (LSR), following Johnson \& Soderblom (1987). We adopted a right-handed coordinate system centered on the Sun, with $U$ toward the Galactic center, $V$ in the direction of Galactic rotation, and $W$ in the direction of the Galactic north pole. Velocities were corrected to the LSR assuming a solar velocity $(U, V, W) \odot$ $=(11.1,12.24,7.25) \mathrm{km} \mathrm{s}^{-1}$ (Schönrich et al. 2010). Uncertainties were propagated through Monte Carlo sampling, assuming normal distributions for all measurement uncertainties. We report the means and standard deviations of these calculations in Table 7.

Figure 10 displays the UVW velocities for our M and L dwarfs and compares them to the $2 \sigma$ velocity spheroids of the Galactic thin disk, thick disk and halo populations as tabulated in Bensby et al. (2003). Most of our sources cluster around $(U, V, W)=(0,0,0)$, with broader dispersions in $U$ and $V$ as compared to $W$. There is also an asymmetric offset in $V$ which correlates with total LSR velocity; this is attributable to asymmetric drift (Strömberg 1924). There is a noticeable trend between $U$ and $V$ among the L dwarfs in our sample, with $U<0$ sources tending to also have $V<0$ and vice versa. We measure a correlation coefficient of $r=0.43 \pm 0.03$; a weaker trend is also seen among the $\mathrm{M}$ dwarfs $(r=0.21 \pm 0.03)$. These trends are discussed in detail below.

We find that the majority of our sources, as expected, fall within the thin disk spheroid, consistent with ages $\lesssim 5$ Gyr. However, several sources fall well outside of this volume. To assess kinematic population membership, we used the method of Bensby et al. (2003) to calculate relative probabilities, $P(\mathrm{TD}) / P(\mathrm{D})$ of membership between the thick (TD) and thin (D) disks.

\footnotetext{
${ }^{6}$ For clarity, all $U V W$ velocities reported in this study are reported in the LSR.
} 
Memberships were assigned as thin disk $(P(\mathrm{TD}) / P(\mathrm{D})<0.1)$, thick disk $(P(\mathrm{TD}) / P(\mathrm{D})>10)$ or intermediate thin/thick disk $(0.1<P(\mathrm{TD}) / P(\mathrm{D})<10)$. The relative probabilities and population assignments are listed in Table 7. Only one source, J0707-4900, falls fully into the thick disk category, while eleven others are intermediate sources, evenly split between late-M and L dwarfs. We also calculated relative probabilities for halo to thick disk membership, but none exceeded 0.002.

\subsection{Velocity Dispersions and Kinematic Ages}

As discussed in Section 1, the total velocity dispersion of a stellar population,

$$
\sigma_{v}^{2} \equiv \sigma_{U}^{2}+\sigma_{V}^{2}+\sigma_{W}^{2}
$$

increases over time as dynamical scattering perturbs Galactic orbits. This produces a correlation between group dispersion and average age. For our analysis, we considered two empirical laws for velocity diffusion. The first is the time-dependent decaying relation of Wielen (1977):

$$
\tilde{\sigma}_{v}(\tau)^{3}=\tilde{\sigma}_{v, 0}^{3}+1.5 \gamma_{v, p} T_{\gamma}\left(e^{\tau / T_{\gamma}}-1\right)
$$

where $\tilde{\sigma}_{v, 0}=10 \mathrm{~km} \mathrm{~s}^{-1}, \gamma_{v, p}=1.1 \times 10^{4}\left(\mathrm{~km} \mathrm{~s}^{-1}\right)^{3} \mathrm{Gyr}^{-1}, T_{\gamma}=5 \mathrm{Gyr}, \tilde{\sigma}_{v}$ is the $|W|$-weighted total velocity dispersion 7 measured in $\mathrm{km} \mathrm{s}^{-1}$, and $\tau$ is the statistical age measured in Gyr. The second is a power-law relation,

$$
\sigma_{v}(\tau)=v_{10}\left(\frac{\tau+\tau_{1}}{10 \mathrm{Gyr}+\tau_{1}}\right)^{\beta}
$$

(cf. Binney \& Tremaine 2008), where $\sigma_{v}$ is the unweighted total velocity dispersion, and we used all six best-fit parameter sets in Aumer \& Binney (2009, Table 2): $55.179 \leq v_{10} \leq 57.975 \mathrm{~km} \mathrm{~s}^{-1}$, $0.148 \leq \tau_{1} \leq 0.261$ Gyr and $0.349 \leq \beta \leq 0.385$.

Table 8 lists the mean velocities, unweighted and weighted dispersions, and corresponding diffusion ages for various subsets of our sample. Uncertainties for all values were propagated forward through Monte Carlo sampling, assuming Gaussian errors for our $U V W$ measurements. For both the full sample ( 85 sources) and 20 pc sample (70 sources), we find nearly identical results, with unweighted total velocity dispersions around $44 \mathrm{~km} \mathrm{~s}^{-1}$ and equivalent ages of $4.8 \pm 0.2 \mathrm{Gyr}$ and 5.2 \pm 0.2 Gyr, respectively, based on the Aumer \& Binney (2009) relation (the Wielen 1977 relation gives similar ages). The derived ages are in good agreement with the radioisotopic age of the Sun, and are generally consistent with mean age estimates for M and L dwarfs from prior studies (e.g., Reid et al. 2002b; Reiners \& Basri 2009; Seifahrt et al. 2010; Blake et al. 2010).

"Wielen (1977) defines the $|W|$-weighted velocity dispersion as having components $\tilde{\sigma}_{x}^{2}=\alpha_{x} \sum_{i}\left|W_{i}\right|\left(X_{i}-\right.$ $\bar{X})^{2} / \sum_{i}\left|W_{i}\right|$ and $\alpha_{x}=\{1,1,0.5\}$ for $x=\{U, V, W\}$. As noted in Reiners \& Basri (2009) and Seifahrt et al. (2010), the weighted dispersion is required to make proper use of the Wielen relations. 


\subsubsection{Spectral Class Variations}

Separating the sample into late-M dwarfs (57 sources) and L dwarfs (28 souces), we find the former to be 1.0-2.5 Gyr younger, depending on the relation used. This result is robust even if the 11 low surface gravity sources, which may be members of YMGs, are rejected (see Section 6): the corresponding ages are 5.0 \pm 0.2 Gyr for late-M dwarfs versus 7.1 \pm 0.4 Gyr for L dwarfs based on the Aumer \& Binney relation. These age differences are statistically significant, and similar to results by Seifahrt et al. (2010) and Blake et al. (2010) who found their L dwarf samples to be kinematically more dispersed than the late-M dwarf samples of Reid et al. (2002b) and Reiners \& Basri (2009). Our identical conclusion with a uniformly-analyzed sample confirms this unexpected trend, which we analyze in detail in Section 5.

We note that the ages inferred between the Wielen (1977) and Aumer \& Binney (2009) relations are discrepant by $2-3 \sigma$ for these two subsamples; the relations produce more significantly discrepant ages for the unusually blue and inactive $\mathrm{L}$ dwarf samples described below. For most subsamples, $\tilde{\sigma} \gtrsim \sigma$, as the inclusion of $|W|$-weighting tends to increase the inferred dispersion for that component. However, for the $\mathrm{L}$ dwarfs, $\tilde{\sigma}_{V}<\sigma_{V}$, driving down the total dispersion and hence Wielen ages. Wielen (1977) included $|W|$-weighting to account for an observed correlation between $V^{2}$ and $|W|$; i.e., the correlation between Galactic orbital eccentricity and inclination. For our M and L dwarfs, we find correlation coefficients $r=0.40 \pm 0.04$ and $0.01 \pm 0.07$ between these parameters; i.e., there is no correlation for the $\mathrm{L}$ dwarfs, which leads to a biased age assessment using the $|W|$-weighting. For this reason, where the inferred ages between the Wielen (1977) and Aumer \& Binney (2009) relations diverge, we favor the latter.

\subsubsection{Color Deviants}

Late M and L dwarfs are known to exhibit broad variations in near-infrared color within a given subtype, variously attributed to surface gravity, metallicity and cloud effects (e.g., Geballe et al. 2002; Knapp et al. 2004; Burgasser et al. 2008; Kirkpatrick et al. 2008; Looper et al. 2008). As noted in Section 1, analysis of the velocity dispersions of color outliers have led to conflicting conclusions as to the relative ages of unusually red and blue M and L dwarfs (Faherty et al. 2009; Schmidt et al. 2010; Kirkpatrick et al. 2010).

We defined color deviants in our sample as having $J-K_{s}$ colors more than 0.15 mag redder or bluer than the median color for their optical spectral type, as delineated in the SDSS samples of Schmidt et al. (2010) and West et al. (2011). The threshhold color offset was chosen because it is comparable to the scatter in median color versus spectral type in these studies, is at least three times the color uncertainties of the vast majority of our sample $\left(\sigma_{J-K_{s}}=0.03-0.04 \mathrm{mag}\right)$, and provides a small but statistically robust sample of outliers (i.e., $10-15 \%$ of the sample). As

$J-K_{s}$ colors generally become more dispersed toward later spectral types (e.g., Kirkpatrick et al. 2008), a constant threshold value may probe more "extreme" M dwarfs as compared to L dwarfs; 
however, as shown below, our L dwarf color outliers appear to be more kinematically distinct. Due to the systematic differences in the colors of our sources compared to the Schmidt et al. (2010) and West et al. (2011) trends (Figure 2), all but one of our unusually red dwarfs (9 sources) are M dwarfs (J0835-0819 is the sole red L dwarf) and all of our unusually blue dwarfs are L dwarfs (11 sources).

For the unusually red dwarfs, we find a kinematic age of 2.0 \pm 0.2 Gyr for the Aumer \& Binney (2009) dispersion relation, considerably younger than the full sample. Remarkably, only one of these red sources, J2045-6332, exhibits Li I absorption and is identified as a low surface gravity dwarf. The remaining sources may have thicker photospheric condensate clouds and/or comprise a coherent, and possibly metal-rich, stream. The unusually blue L dwarfs, on the other hand, have a large velocity dispersion $\left(61 \mathrm{~km} \mathrm{~s}^{-1}\right)$ and dispersion age of $12.4 \pm 0.9 \mathrm{Gyr}$ (for the Wielen 1977 relation the dispersion age is a more reasonable $7.0 \pm 0.2 \mathrm{Gyr}$ ). Roughly $45 \%$ of these sources are also identified as intermediate thin/thick disk stars. The fact that the kinematically colder red dwarfs are mostly type $\mathrm{M}$ and the kinematically warmer blue dwarfs are all of type $\mathrm{L}$ provides a possible explanation for the age offsets between these spectral classes. Indeed, when color deviants are excluded, the kinematic ages of the late-M dwarfs (4.0 $\pm 0.2 \mathrm{Gyr})$ and L dwarfs $(3.4 \pm 0.3)$ conform to expectations from population simulations (see below). Thus, we have evidence that the age discrepancy between late-M and L dwarfs originates as a color discrepancy. Section 5.2 discusses this insight in further detail.

\subsubsection{Magnetically Active and Inactive Dwarfs}

Nonthermal magnetic emission is a common metric for low-mass stellar ages, as emission declines in strength as stars spin down through wind-driven angular momentum loss (e.g., Wilson 1963; Skumanich 1972; Soderblom et al. 1993; Fleming et al. 1995). This process appears to be less efficient for late-M and L dwarfs based on the longer timescales inferred for magnetic field decay (West et al. 2006; Schmidt et al. 2007; West et al. 2008; Reiners \& Basri 2008) and faster rotation rates among older L dwarfs (Mohanty \& Basri 2003; Reiners \& Basri 2006, 2010). There has also been evidence of a reversal in the standard age-activity relation in the L dwarf regime, with only the older and more massive stellar L dwarfs having sufficient field energy to drive nonthermal emission (Gizis et al. 2000; Reiners \& Christensen 2010; Burgasser et al. 2011).

As discussed in Section 2.4, while the majority of our sample exhibits $\mathrm{H} \alpha$ emission, the $\mathrm{L}$ dwarfs are roughly evenly split between active and inactive sources. Comparing these subsets, we find the that active $\mathrm{L}$ dwarfs have a significantly smaller overall dispersion $\left(45.9 \pm 1.1 \mathrm{~km} \mathrm{~s}^{-1}\right)$ than the non-active $\mathrm{L}$ dwarfs $\left(51.5 \pm 0.7 \mathrm{~km} \mathrm{~s}^{-1}\right)$ and a correspondingly younger dispersion age (5.6 \pm 0.4 Gyr versus 7.8 \pm 0.5 Gyr for the Aumer \& Binney relation). This would appear to confirm an underlying age-activity relation that is similar to earlier-type stars; i.e., older L dwarfs are less active. 


\subsubsection{Li-bearing and Low Surface Gravity Brown Dwarfs}

The nine late $\mathrm{M}$ and $\mathrm{L}$ dwarfs exhibiting Li I absorption exhibit the smallest velocity dispersions in our sample, with $\sigma_{v}=10.4 \pm 0.5 \mathrm{~km} \mathrm{~s}^{-1}$. Both age dispersion relations are undefined for this value, yielding effective upper limits of order $100 \mathrm{Myr}$. This is qualitatively in line with evolutionary model predictions from Baraffe et al. (2003), as a Li-bearing M7.5 (versus L1.5) dwarf with an estimated $8 \mathrm{~T}_{\text {eff }} \approx 2600 \mathrm{~K}$ ( versus $2050 \mathrm{~K}$ ) and mass below $0.06 \mathrm{M}_{\odot}$ should have an age of no more than $120 \mathrm{Myr}$ ( versus $570 \mathrm{Myr}$ ). Several of these sources show low surface gravity features in their optical spectra (Section 2.5) and are kinematic members of nearby young moving groups with ages of 10-100 Myr (Section 5). Hence, the velocity dispersions are consistent with overall spectral properties. Similarly, the total velocity dispersion for the 11 low surface gravity sources is $11.9 \pm 0.6 \mathrm{~km} \mathrm{~s}^{-1}$, again implying ages $\lesssim 100$ Myr.

\subsection{Velocity Probability Distributions}

The ages estimated in the previous section are contingent on the velocity distributions being Gaussian. However, both visual examination of the $U V W$ plots in Figure 10 and discrepancies between the age-dispersion relations used argue that non-Gaussian effects are likely present (see also Binney et al. 2014). We therefore constructed probability ("probit") plots for our various subsamples for each of the $U, V$ and $W$ coordinates, following procedures described in previous studies (Lutz \& Upgren 1980; Reid et al. 2002a; Bochanski et al. 2007a; Reiners \& Basri 2009). Probit plots are a rank order mapping of velocity to Gaussian probability, generating a straight line for a single Gaussian distribution with a slope equal to the standard deviation. Deviations from Gaussian emerge as variations in the slope.

Figure 1113 displays $U V W$ probit plots for our 20 pc, late-M dwarf and L dwarf subsamples. It is clear that only the $U$ distributions for the $20 \mathrm{pc}$ and late-M dwarf samples are single Gaussian distributions; both $V$ and $W$ distributions show significant slope variations beyond $\pm 1 \sigma$, with those in $W$ being more pronounced. Following Bochanski et al. (2007a), we performed a piece-wise fit to these trends, over "core" $(|\sigma| \leq 1)$ and "wing" components $(|\sigma|>1)$, sampling the data uncertainties through Monte Carlo analysis. Table 8 lists the resulting $U V W$ and total velocity dispersions (unweighted) and corresponding ages based on the Aumer \& Binney (2009) relation. In general, core components have dispersions and ages just below those of the full sample analysis above, while the wing components have greater dispersions and older kinematic ages. The $V$ probit plots exhibit extended tails to negative velocities and curvature at positive velocities, which again can be attributed to asymmetric drift.

Focusing on the M and L dwarf subsamples, we find clear differences in the velocity distri-

\footnotetext{
${ }^{8}$ Based on the $\mathrm{T}_{\text {eff }}$ /spectral type relation of Stephens et al. (2009).
} 
butions for all three components, most notably in $U$. Here, the $\mathrm{L}$ dwarfs exhibit both a nonzero mean velocity $\left(\langle U\rangle=14.7 \pm 0.5 \mathrm{~km} \mathrm{~s}^{-1}\right)$ and a pronounced asymmetry about this mean. The offset indicates a net flow of L dwarfs toward the Galactic center, and persists even when color deviants are excluded $\left(\langle U\rangle=10.1 \pm 0.8 \mathrm{~km} \mathrm{~s}^{-1}\right)$. The slope change in the probit plot across the mean is not seen in any of the other velocity components or subsamples. This pattern is remarkable and, along with the correlation between $U$ and $V$ and lack of correlation between $V^{2}$ and $|W|$, suggests that the kinematics of L dwarfs in our sample are distinct, either intrinsically or though sample bias. We focus on this problem in Section 5.

\subsection{Galactic Orbits}

As a final examination of the statistical properties of our sample, we used the $U V W$ velocities and Galactocentric coordinates to compute Galactic orbits in a static, axisymmetric potential. We followed the same strategy as described in Bochanski et al. (2011), converting heliocentric velocities to cylindrical velocities $\left(V_{R}, V_{\phi}, V_{Z}\right)$ in the Galactic frame of rest, assuming an LSR azimuthal motion of $240 \mathrm{~km} \mathrm{~s}^{-1}$ (Reid et al. 2014; Antoja et al. 2015). We computed Galactic spatial coordinates for our sources relative to an assumed solar position of $(X, Y, Z)_{\odot}=(-8.43,0$, 0.027) kpc (Chen et al. 2001; Reid et al. 2014), with $X Y Z$ defined in the same manner as $U V W$. For the Galactic potential, we adopted static, axisymmetric oblate Plummer's sphere models for the Galactic halo, bulge and disk, using the forms described in Kuzmin (1956) and Mivamoto \& Nagai (1975), with parameters from Dauphole \& Colin (1995). A fourth-order Runge-Kutta integrator was used to calculate the orbit over a period of $\pm 250 \mathrm{Myr}$ about the current epoch in $10 \mathrm{kyr}$ steps, and both energy and the $Z$-component of angular momentum were conserved to better than one part in $10^{-3}$. To sample measurement uncertainties, we computed 100 orbits for each source, varying the initial distances and velocities in a Monte Carlo fashion assuming normal distributions scaled to the uncertainties listed in Tables 1 and 7.

Figure 14 displays the distributions of inferred orbital elements for our sample: minimum and maximum Galactic radius, maximum absolute vertical displacement, eccentricity $\left(e \equiv\left[R_{\max }-\right.\right.$ $\left.\left.R_{\text {min }}\right] /\left[R_{\max }+R_{\min }\right]\right)$, and maximum inclination $\left(\tan i \equiv Z / \sqrt{X^{2}+Y^{2}}\right)$. As expected for a sample dominated by the thin disk population, the majority of our sources exhibit circular $(\mathrm{e} \lesssim 0.15)$ and planar orbits $\left(i \lesssim 2^{\circ}\right)$, although the core of the eccentricity distribution extends to 0.2. Figure 15 shows the orbits of the two sources with the largest values of $P(\mathrm{TD}) / P(\mathrm{D})$, the M8.5 J0707-4900 and the L1 J0921-2104. Both have fairly eccentric orbits $(e=0.4)$ which carry them to perigals just outside the spherical bulge $(\mathrm{R} \approx 4 \mathrm{kpc}$; Binney \& Tremaine 2008), suggesting that they may be scattered bulge stars. In contrast, several sources currently near perigal reach Galactic radii of over 14 kpc (e.g., the D/TD dwarfs J1539-0520 and J2331-2749). Our local VLM dwarf population therefore samples a significant region of the Galaxy, a point returned to in Section 5. Note that all of the orbits are prograde, consistent with formation in the disk (e.g., Carollo et al. 2008).

Given the distinct velocity distributions of the $\mathrm{M}$ and $\mathrm{L}$ dwarfs in our sample, we examined 
whether their Galactic orbits differed as well. Figure 16 compares the distributions of minimum and maximum Galactic radii for these two subsamples. The L dwarfs have a broader distribution of Galactic radii, with a notable skew to higher maximum radii. Median values for $R_{\max }$ for $\mathrm{M}$ and L dwarfs are $9.5 \mathrm{kpc}$ and $10.3 \mathrm{kpc}$, respectively; while median eccentricities are 0.11 and 0.16 . In contrast, the median values of $\left|Z_{\max }\right|$ and orbital inclination are the same for both groups. It appears that the L dwarfs in our sample are distributed more broadly in Galactocentric radius than the late-M dwarfs, which is directly attributable to their unusual $U$ velocity distribution.

\section{Discussion: Why are Local L Dwarfs Blue and Dispersed?}

The confirmation that nearby L dwarfs appear to be, on average, more dispersed and kinematically older than nearby late-M dwarfs, and that this dispersion is driven by a large fraction of unusually blue L dwarfs, suggests that there is something unusual about the local L dwarf population. Most notable is the asymmetric $U$ distribution of this population that remains even when color outliers are rejected, and indicates the existence of a net radial flow of $\mathrm{L}$ dwarfs that is not matched by the late-M dwarfs. Asymmetries in the radial motions of local stars have been observed, and are generally attributed to resonances with Galactic structures; e.g., the Galactic bar and/or spiral arm patterns (Dehnen \& Binnev 1998; Dehnen 2000; Sellwood \& Binnev 2002; De Simone et al. 2004; Quillen \& Minchev 2005; Famaev et al. 2007; Minchev \& Famaey 2010; Quillen et al.|2011). Recent large-scale RV surveys, most notably the RAdial Velocity Experiment (RAVE; Steinmetz et al. 2006; Siebert et al. 2011; Williams et al. 2013; Binney et al. 2014; Sharma et al. 2014) have measured statistically significant RV gradients of order $3-10 \mathrm{~km} \mathrm{~s}^{-1} \mathrm{kpc}^{-1}$ directed toward the Galactic center. Antoja et al. (2015) also find that thin disk stars in the RAVE survey $([\mathrm{M} / \mathrm{H}] \geq-0.1,|Z|<$ $0.5 \mathrm{kpc})$ exhibit a correlation between radial and azimuthal motions, with trailing sources $\left(V_{\phi}<0\right)$ streaming outward $\left(V_{r}<0\right)$ and leading sources $\left(V_{\phi}>0\right)$ streaming inward $\left(V_{r}>0\right)$. They attribute this correlation to the local Outer Lindblad Resonance with the Galactic bar, which also

builds the Hercules stream (Kalnajs 1991; Dehnen 2000; Antoja et al. 2014). We see precisely this same trend among the L dwarfs in their $U$ and $V$ velocities (Figure 10), but curiously not among the late-M dwarfs.

Given prior evidence of radial motion gradients among local stars, we hypothesize that the variance between the late-M and L dwarfs in our sample arises from two possible sources: (1) an inherent asymmetry in the ages and velocity distribution of local $\mathrm{L}$ dwarfs made manifest by brown dwarf thermal evolution; and (2) a bias among the L dwarfs in our sample or in the local $20 \mathrm{pc}$ population. We consider each of these hypotheses in turn. 


\subsection{Is There an Inherent Asymmetry in L Dwarf Ages and Kinematics?}

The lowest-order inherent asymmetry in the distribution of stars in the Galactic disk is the radial density distribution, which increases toward the Galactic center. To test whether this spatial asymmetry, coupled with brown dwarf evolution, could drive an asymmetry in the radial motions of L dwarfs, we performed a Monte Carlo population simulation combining brown dwarf evolution, age-dependent velocity dispersions, radial mixing through Galactic orbital motion, and selection biases inherent to a local sample.

A population of stars and brown dwarfs was generated as described in Burgasser (2004, 2007), assuming an initial mass function

$$
\frac{d N}{d M} \propto M^{-\alpha}
$$

and age distribution (star formation rate)

$$
\frac{d N}{d \tau} \propto e^{\beta\left(\tau-T_{0}\right)} .
$$

Here, $M$ is mass, constrained to $0.01 \mathrm{M}_{\odot} \leq M \leq 0.20 \mathrm{M}_{\odot} ; \tau$ is age, constrained to 0.2 Gyr $\leq \tau \leq$ $8 \mathrm{Gyr} ; N$ is the number density of stars in a given volume; $\alpha=\{-0.5,0.0,0.5,1.0\}$ is the mass function power-law index; $\beta=\{-0.5,0.0,0.5,1.0\}$ is the star formation rate power-law index; and $T_{0}=8$ Gyr was adopted as the oldest age of stars in the Solar Neighborhood (Haywood et al. 2013). Note that $\alpha>0$ yields a population dominated by lower-mass objects $(\alpha \approx 0.5$ in nearby clusters; see Bastian et al. 2010 and references therein), while $\beta>0$ yields a population dominated by older objects $(\beta=0$ is a common assumption for Galactic population simulations, $\beta=1$ is consistent with the integrated star formation rate history of field galaxies; see Madau et al. 1998; Aumer \& Binney 2009). Drawing $10^{5}$ values of $M$ and $\tau$ from these distributions, we inferred the present-day effective temperatures of each source using the evolutionary models of Burrows et al. (2001), and converted these to spectral types using the empirical relation of Stephens et al. (2009). We limited our analysis to those sources with spectral types between M7 and L5, which represents $6-17 \%$ of the original simulation sample depending on $\alpha$ and $\beta$. We did not consider the role of multiplicity.

Asymmetry in the Galactic stellar distribution and radial mixing were implemented by assigning initial Galactic radii in the range $5.5-11.5 \mathrm{kpc}$, drawing from a radial exponential distribution

$$
\frac{d N}{d R} \propto e^{\left(R_{\odot}-R\right) / L}
$$

where $R$ is the radial coordinate, $R_{\odot}=8.43 \mathrm{kpc}$ is the solar Galactic radius (Reid et al. 2014), and

$L=2.1 \mathrm{kpc}$ is the radial scaleheight (Jurić et al. 2008). We then assigned $U V W$ velocities in the LSR based on normal distributions centered on zero and with standard deviations determined from the assigned age and the Aumer \& Binney (2009) age-dispersion relations above. We also included an asymmetric drift term

$$
V_{a}=\frac{\sigma_{R}^{2}}{74 \mathrm{~km} / \mathrm{s}}=23.7\left(\frac{\tau}{10 \mathrm{Gyr}}\right)^{0.614} \mathrm{~km} / \mathrm{s}
$$


based on Aumer \& Binney (2009). The assigned velocities and initial Galactic coordinates (assuming $Y=Z=0$ ) were used as initial conditions to compute orbits over 500 Myr in steps of 1 Myr in an axisymmetric potential as described in Section 4.4. From these orbit calculations we identified all timesteps among all sources for which $\left|R-R_{\odot}\right| \leq 50 \mathrm{pc}$ and $|Z| \leq 50$ pc. These 7,000-30,000 orbital snapshots (the number depending on simulation parameters) comprised our "local" sample, and included multiple instances of sources which repeatedly fell within the Galactic Solar torus.

Figure 17 summarizes the results of our baseline simulation with $\alpha=0.5$ and $\beta=0.0$, comparing distributions between the initial simulation sample to the dynamically-evolved and locallyselected sample. Independent of selection mechanism, these calculations affirm prior results that the $\mathrm{L}$ dwarf population should be on average $0.2-0.4$ Gyr younger than the late-M dwarf population due to the loss (through thermal evolution) of old brown dwarfs. This confirms the results for our "normal" color populations, but not the full sample of late-M and L dwarfs. Dynamical evolution and local selection results in a uniformly younger "observed" population, with both late-M and L dwarfs being 0.3-0.6 Gyr younger than the initial simulation sample. This offset stems from preferential selection of young objects originating near $\mathrm{R}_{\odot}$, while most of the older objects scatter outward and are not fully replaced by older objects scattered into $\mathrm{R}_{\odot}$. We note that the mean ages of locally-selected late-M and L dwarfs in this baseline simulation are somewhat younger than the ages inferred from our velocity analysis; indeed, a value of $\beta$ between 0.0 and 0.5 appears to be more consistent with the data, suggesting a decline in the VLM star/brown dwarf formation rate over the age of the Galaxy. Nevertheless, we see no evidence of a distinct, highly-dispersed population of $\mathrm{L}$ dwarfs for any of the simulation parameters examined.

To produce a sample in which $\mathrm{L}$ dwarfs are on average older than late-M dwarfs, we explored cases where the star formation history differed between stars and brown dwarfs. A divergent formation history could arise from mass-dependence in the Galactic birth rate. Figure 18 shows the results for a simulation assuming $\beta=0.5$ for brown dwarfs $\left(\mathrm{M}<0.07 \mathrm{M}_{\odot}\right)$ and $\beta=0.0$ for stars $\left(\mathrm{M}>0.07 \mathrm{M}_{\odot}\right)$; we also considered the opposite $\beta$ assignments. The simulation with older brown dwarfs does indeed produce a more dispersed and kinematically older population of $\mathrm{L}$ dwarfs by 0.4-0.7 Gyr, although the mean dispersion ages are again younger for both M and L dwarfs than those observed in our full sample.

One possible resolution to the divergent results between simulated and observed kinematic dispersion ages is error in the evolutionary models used to predict the timescales for brown dwarf cooling in our simulations. If the cooling rates are slower than these models predict, we would expect VLM sources to remain L dwarfs for longer periods of time, bringing the simulations into agreement with our observations. Indeed, recent observations of L-type binaries with orbital mass measurements have shown that these sources are 60-100\% more luminous than models predict (Dupuy et al. 2009a; Konopacky et al. 2010; Dupuy et al. 2014), so this is a valid concern for our simulations.

The simulations generated two clear asymmetries in the spatial and velocity properties of late- 
$\mathrm{M}$ and L dwarfs. First, the majority of locally-selected dwarfs in all of the simulations originate (or are at least initially placed) within the Solar radius, with an average offset of $\sim 1 \mathrm{kpc}$. This largely reflects the assumed exponential decline in stellar density with Galactic radius. Second, the azimuthal velocity distributions are skewed to negative velocities, reflecting both the initial radial distribution of stars (which lose azimuthal speed as they climb out of the Galactic potential) and our input azimuthal drift term. However, there are no significant differences between the late-M and L dwarfs for these two parameters, nor for radial and vertical velocity distributions, which are symmetric about and centered on zero. This is true even for separate values of $\beta$ between stars and brown dwarfs. It appears that we cannot reproduce Galactic radial velocity asymmetries with an axisymmetric potential, as individual stars passing through the local volume have nearly the same probability of moving radially inward as outward on this scale.

Hence, while an older population of $\mathrm{L}$ dwarfs can be produced with an assumption of different star formation rates between stars and brown dwarfs, or may be reproduced if there are errors in the evolutionary models; an inherent asymmetry in the $U$ velocity distribution of $\mathrm{L}$ dwarfs appears to require perturbations from a non-axisymmetric source; i.e., the Galactic bar and spiral structure. Analysis of these hypotheses are left to more detailed simulations in a future publication, although it is important to assert that any radial mixing induced by Galactic structure must influence M and L dwarfs differently to match our results and those of Seifahrt et al. (2010) and Blake et al. $(2010)$.

\subsection{Is There Sample or Cosmic Bias in the Local L Dwarf Population?}

A more mundane explanation for our results is that the sample considered here is kinematically biased in its construction. The sample was drawn primarily from all-sky imaging and proper motion surveys, which continue to be incomplete in the Solar Neighborhood (e.g., Luhman 2013; Scholz 2014; Pérez Garrido et al. 2014). Incompleteness is particularly an issue along the Galactic plane due to source crowding. For our sample, we also have a declination limit imposed by the observing site $\left(\delta<25^{\circ}\right.$; Figure 1). To assess whether these "pointing" asymmetries produce velocity trends, Figure 19 displays the distributions of $X Y Z$ coordinates for our sample. The $\mathrm{M}$ and $\mathrm{L}$ dwarfs have similar distributions in $X$ and $Y$, but in $Z$ the $\mathrm{L}$ dwarfs are more centrally concentrated with a slight bias toward positive $Z$ (both subsamples have fewer sources at $Z=0$ due to Galactic plane exclusion). However, our simulations show no correlation between local $Z$ position and $U$ velocity; simulations by Faure et al. (2014) which include spiral perturbations are similarly symmetric about the Galactic plane. It is therefore unclear what role this difference in vertical spatial distribution would play in producing an asymmetric radial velocity distribution.

Another possible bias is the contribution of YMG members in our sample. At least 8 of the sources investigated here are kinematically associated with YMGs, 7 of which are late-M dwarfs (Section 6). Similarily, 9 of the 11 low surface gravity dwarfs and 8 of the 9 sources exhibiting Li I absorption are late-M dwarfs. There is clearly a "youth bias" between the M and L dwarf 
subsamples. However, as noted in Section 4.2.1, while rejecting sources which exhibit low surface gravity features slightly increases the velocity dispersion for the late-M dwarfs and brings their kinematic age closer to (but still less than) the L dwarfs, editing out the low gravity sources does not change the underlying $U$ velocity distributions. Late-M dwarfs remain symmetrically distributioned about $U=0$, and $\mathrm{L}$ dwarfs asymmetric and offset. Indeed, removal of the two low gravity L dwarfs in our sample increases the mean velocity offset of the remainder. Hence, contamination by YMGs does not appear to resolve the velocity differences between the M and L dwarfs.

A third possibility is that the local sample itself has an inherent cosmic bias. As described in Section 4.2.2, the unusually blue dwarfs, which are all $\mathrm{L}$ dwarfs, are far more dispersed than the unusually red dwarfs, which are predominantly late-M dwarfs. The unusually blue $\mathrm{L}$ dwarfs represent $39 \%$ of all the $\mathrm{L}$ dwarfs in our sample. This suggests a selection effect. However, the identification of color deviants is based on mean near-infrared colors from the optically-selected SDSS surveys, which as discussed in Schmidt et al. (2010) are less color-biased than 2MASS samples and tend to identify bluer sources. It is therefore remarkable that a plurality of the L dwarfs examined here, mostly identified in the 2MASS survey, are bluer still. Evidence that this color skew may actually be a local effect emerges from the fact that 10 of the 11 unusually blue $\mathrm{L}$ dwarfs are within 20 pc (the exception is J0923+2340), which is a higher fraction (91\%) than the remainder of the $\mathrm{L}$ dwarf sample $(65 \%)$. In other words, the unusually blue $\mathrm{L}$ dwarfs are more representative of the local volume than the "normal-color" L dwarfs delineated in Schmidt et al. (2010). It is possible that we are seeing two distinct populations of $\mathrm{L}$ dwarfs in the 20 pc volume: a "disk" group whose dispersions conform to simulation expectations, and a "dispersed" group drawn from an older, possibly thick disk VLM population. Given the relatively small number of L dwarfs examined in this study, this speculative hypothesis must be confirmed through a larger study.

\section{Candidate Kinematic Members of Nearby Moving Groups and Associations}

Several of our sources exhibit Li I absorption and/or spectral features indicative of low surface gravity, and as such are potential members of YMGs. To assess which YMGs these sources are affiliated with, and their probability of affiliation, we used the Bayesian Analysis for Nearby Young AssociatioNs II tool (BANYAN II; Gagné et al. 2014) which uses spatial and velocity coordinates and photometry to assess the probability of membership $\left(P_{M}\right)$ and field contamination $\left(P_{C}\right)$ for individual sources. Our YMG sample included the TW Hydrae Association (TWA; Kastner et al. 1997; de la Reza et al. 1989, Zuckerman \& Song 2004; 10 Myr; Weinberger et al. 2013), the $\beta$ Pictoris Moving Group ( $\beta$ PMG; Zuckerman et al. 2001; 20 - 26 Myr; Mamajek \& Bell 2014, Malo et al. 2014, Binks \& Jeffries 2014), the Tucana-Horologium Association (THA; Torres et al. 2000, Zuckerman \& Webb 2000; 40 Myr; Kraus et al. 2014), the Carina association (CAR; 20 - 40 Myr; Torres et al. 2008), the Columba association (COL; 20 - 40 Myr; Torres et al. 2008), the Argus/IC 2391 association (ARG;

30 - 50 Myr; Torres et al. 2008), and the AB Doradus moving group (ABD; Zuckerman et al. 2004; 
110 - 130 Myr ; Luhman et al. 2005, Barenfeld et al. 2013). We adopt the spatial and kinematic models for each of these associations given in Gagné et al. (2014), and include distances in our comparison for those sources with trignometic parallax measurements.

Table 10 lists the membership and contamination probabilities of sources with membership probabilities $P_{M}>10 \%$. We also list the spatial $(\Delta D)$ and velocity $(\Delta V)$ offsets from the respective centers of the best-match association. In the following discussion on individual candidates, we used the effective temperature $\left(\mathrm{T}_{\text {eff }}\right)$ /spectral type calibration of Stephens et al. (2009) and the evolutionary models of Baraffe et al. (2003) to estimate physical parameters.

\subsection{Previously Known Candidate Members}

J0041-5621AB (M6.5 + M9): The combined-light spectrum of this resolved binary displays $\mathrm{H} \alpha$ emission, Li I absorption, and weak low surface gravity features. It was previously identified as a possible member of either THA or $\beta$ PMG by Reiners \& Basri (2009) on the basis of its Li I absorption, kinematics and evidence of ongoing accretion. Gagné et al. (2014) favored association with THA based on a BANYAN II analysis of the same data, and our revised analysis supports that conclusion, with a membership probability $P_{M}>99.9 \%$ and $P_{C}<0.1 \%$. While this source does not have a trigonometric parallax, the BANYAN II tool predicts a statistical distance of $41_{-3}^{+2} \mathrm{pc}$ if it is a member of THA, placing it $7 \mathrm{pc}$ and $1.4 \mathrm{~km} \mathrm{~s}^{-1}$ away from the center of the spatial and kinematic model. Reiners et al. (2010) resolved the system into a $142.8 \pm 0.5$ mas binary and estimated component types of M6.5+M9 and masses of $30 M_{\mathrm{Jup}}$ and $15 M_{\mathrm{Jup}}$ for an age of 10 Myr. Our combined-light spectrum is considerably later than M6.5 (Figure 7), suggesting that the primary may be cooler than inferred in that study. Adopting effective temperatures of $2500 \mathrm{~K}$ and $2400 \mathrm{~K}$ based on component types of M8+M9, a THA age of $40 \mathrm{Myr}$, a distance of $41 \mathrm{pc}$, and the evolutionary models of Baraffe et al. (2003), we predict masses of $40 M_{\mathrm{Jup}}$ and $35 M_{\mathrm{Jup}}$, a projected separation of $6 \mathrm{AU}$, and an orbit period of $50 \mathrm{yr}$. The last value is less than half that estimated by Reiners et al. (2010), and suggests that orbital motion could be detectable over the coming decade.

J0123-6921 (M9) was proposed as a THA member by Gagné et al. (2014), and our reanalysis supports this conclusion $\left(P_{M}>99.9 \%\right.$ and $\left.P_{C}<0.1 \%\right)$. Like J0041-5621AB, this source exhibits Li I absorption, $\mathrm{H} \alpha$ emission and weak signatures of low surface gravity. We again infer a signficantly later spectral type for this source than the M7.5 reported in Reiners \& Basri (2009), and we estimate its mass to be $35 M_{\mathrm{Jup}}$.

J0339-3525 (LP 944-20, M9) has been identified by Ribas (2003) as a candidate member of the controversial Castor "association" (CAS; Barrado y Navascues 1998). In contrast, Gagné et al. (2014) identify J0339-3525 was a candidate member of ARG using BANYAN II, but note that its $X Y Z U V W$ coordinates are much closer to CAS. Our findings show a weak membership probability for $\operatorname{ARG}\left(P_{M}=16.7 \%\right)$ but low field contamination $\left(P_{C}=0.3 \%\right)$, suggesting that it is likely 
part of a different association. The spectrum of J0339-3525 shows weak features of low surface gravity, with particularly enhanced VO absorption at $7400 \AA$ and weak and narrow alkali lines, $\mathrm{H} \alpha$ emission, and weak Li I absorption (Tinnev 1998; Reiners \& Basri 2009). All of these observations point to either a very young $(\lesssim 30 \mathrm{Myr})$ low-mass brown dwarf or, as argued by Tinney (1998), Ribas (2003) and Pavlenko et al. (2007) an "intermediate"-aged ( $\approx 300-600$ Myr) brown dwarf with partial Li depletion. It has been recently argued that hypothesized CAS "members", which

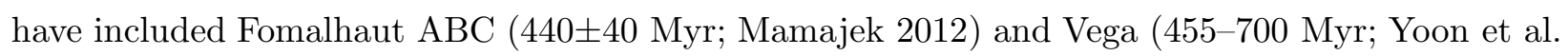
2010; Monnier et al. 2012), do not share a common origin or composition, and is likely a dynamical stream (e.g., Mamajek et al. 2013; Mamajek 2012; Monnier et al. 2012; Zuckerman et al. 2013). Indeed, Mamajek et al. (2013) specifically note that J0339-3525's current proximity to Fomalhaut may be short-lived. J0339-3525 may therefore be a "juvenile" brown dwarf caught up in a Galactic dynamical pattern rather than a member of a bona-fide association.

J1139-3159 (TWA 26, M9 $\gamma$ ) is a previously reported member of TWA (Gizis 2002), and our results are consistent with this assignment $\left(P_{M}=99.6 \%\right.$ and $\left.P_{C}=0.1 \%\right)$. With strong signatures of low surface gravity, Li I absorption and a full complement of $\mathrm{H} \alpha, \mathrm{H} \beta$ and $\mathrm{H} \gamma$ emission, this source is an unambiguous young brown dwarf.

J2000-7523 (M9) is a previously reported candidate of $\beta$ PMG (Gagné et al. 2014) or CAS (Gálvez-Ortiz et al. 2010) with pronounced low surface gravity features. Our BANYAN analysis favors the $\beta$ PMG assignment $\left(P_{M}=99.0 \%, P_{C}=4.1 \%\right)$. Notably, this source does not show Li I absorption, an effect likely related to weak alkali lines in low surface gravity photospheres as posited by Kirkpatrick et al. (2008). That study suggests an age of $\lesssim 30$ Myr for such sources, which is consistent with $\beta$ PMG membership.

J2045-6332 (M9) was identified in Gálvez-Ortiz et al. (2010, 2014) as a high-probability member of CAS (note discussion above). We confirm the presence of Li I absorption reported in those studies, and also note $\mathrm{H} \alpha$ and $\mathrm{H} \beta$ in emission and strong low surface gravity spectral features, implying a young source. Like J2000-7523, we find this source to have a better membership match to $\beta$ PMG $\left(P_{M}=87 \%, P_{C}=0.2 \%\right)$, which is also supported by its strongly suppressed alkali lines, although the peculiar motion is somewhat large $\left(7 \mathrm{~km} \mathrm{~s}^{-1}\right)$.

\subsection{New Candidate Members}

J0823-4912AB (L3) is a low-probability candidate member of $\beta$ PMG based on our analysis $\left(P_{M}=30 \%, P_{C}=0.4 \%\right)$. This source exhibits both Li I absorption and spectral features consistent with low/intermediate surface gravity. Its distance and proper motion measurements give it relatively large peculiar motion relative to $\beta$ PMG $\left(7 \mathrm{~km} \mathrm{~s}^{-1}\right)$. Sahlmann et al. $(2013,2015)$ have identified this source as an astrometric binary with a mid-to-late L dwarf companion, and it is possible that the orbital motion of this system is skewing the inferred systemic motion in the radial direction (tangential motion is likely averaged out). Sahlmann et al. (2015) also report Li I in 
absorption and the presence of low-surface gravity features in its near-infrared spectrum. However, based on comparison of the system mass function, component classifications and evolutionary models, the Sahlmann et al. (2015) study supports an age in the range 70-470 Myr for J0823-4912AB, considerably older than $\beta$ PMG. J0823-4912AB may therefore be an interloping member of a older YMG or stream, perhaps CAS.

J1510-2818 (M9) is identified as a modest-probability candidate member of ARG $\left(P_{M}=60 \%\right.$ and $P_{C}=34 \%$ ). The source exhibits $\mathrm{H} \alpha$ emission and strong signatures of low surface gravity in its spectrum, but no Li I. Again, the absence of Li I may be related to weak surface gravity at an age $\lesssim 30 \mathrm{Myr}$, which is marginally consistent with the 30-50 Myr age estimate for ARG. A trigonometric distance measurement could confirm or refute ARG membership; BANYAN II predicts a statistical distance of $27_{-3}^{+2}$ pc for ARG membership, and $35_{-7}^{+11}$ pc for membership in the field.

\subsection{Interlopers}

J1456-2809 (LHS 3003, M7) emerged as a candidate member of ABD in our analysis $\left(P_{B}=\right.$ $\left.94.3 \%, P_{C}=4.3 \%\right)$. However, this source displays no signs of low surface gravity in either its optical or near-infrared spectra (Bardalez Gagliuffi et al. 2014), and no Li I absorption, implying an age $\gtrsim 500 \mathrm{Myr}$. Its $\mathrm{H} \alpha$ emission does not provide a good constraint on its age; chromospheric activity lasts up to $8 \mathrm{Gyr}$ in late-M dwarfs (West et al. 2011). With its close proximity to the locus of ABD members (21.2 pc and $2.5 \mathrm{~km} \mathrm{~s}^{-1}$ from the center of our model), J1456-2809 highlights the importance of identifying signs of youth before assigning YMG membership.

\subsection{Candidate Young Brown Dwarfs Not Assigned to a Young Moving Group}

Three additional sources exhibiting spectral signatures of low surface gravity were not found to be members of the well-characterized young associations listed above. We performed a secondorder check of additional systems based exclusively on $U V W$ velocities: the Octans Association $(\approx 30-40$ Myr; Murphy \& Lawson 2015); the Ursa Major Moving Group ( $\approx 500$ Myr; King et al. $2003)$; the Hercules-Lyrae Moving Group ( $\approx 250$ Myr; Eisenbeiss et al. 2013); Carina-Near Moving Group ( $\approx 200 \mathrm{Myr}$; Zuckerman et al. 2006); and various streams listed in Zuckerman \& Song (2004), including CAS.

J0652-2534 (M9) has a spectrum similar to J0339-3525, with somewhat weakened alkali absorption (including narrow K I doublet lines) and enhanced VO absorption at $7900 \AA$, both suggesting a modestly low surface gravity. In addition, the spectrum exhibits Li I absorption, but no $\mathrm{H} \alpha$ emission. Given these features, J0652-2534 is likely a few hundred Myr old. Its closest match in $U V W$ space is Octans, but the source is about $20 \mathrm{~km} \mathrm{~s}^{-1}$ discrepant from that association's $U V W$ center and is probably too old. This "juvenile" brown dwarf must be an member of an association 
or stream not considered here, or may be an unassociated system.

J0909-0658 (L1) exhibits strong signatures of low surface gravity, most notably enhanced VO absorption at $7400 \AA$ and $7900 \AA$, weak FeH and $\mathrm{CrH}$ bands, and somewhat narrower K I lines. This source does not exhibit Li I absorption, and the presence of clear Na I, Rb I and Cs I lines argues against low surface gravity being the reason. Assuming a mass greater than $0.06 \mathrm{M}_{\odot}$ and $\mathrm{T}_{\text {eff }}=2100 \mathrm{~K}$, this implies an age of at least $500 \mathrm{Myr}$. Of all the kinematic groups listed above, ARG has the closest association in $U V W$ space, but J0909-0658 is clearly too old for that group. If this source is a young brown dwarf, it must be a member of an older association or stream or solivagant. Alternately, its unusual metal-oxide and metal-hydride features may reflect nonsolar (possibly super-solar) composition. Thorough analysis of this source's full spectral energy distribution is warranted.

J1411-2119 (M9), like J0339-3525 and J0652-2534, exhibits weak spectral signatures of low surface gravity (weak Na I absorption at $8183 / 8195 \AA$ ) and Li I absorption, as well as pronounced $\mathrm{H} \alpha$ and $\mathrm{H} \beta$ emission. Its closest match in $U V W$ space is the $\approx 500$ Myr Ursa Majoris Group, but J1411-2119 remains about $13 \mathrm{~km} \mathrm{~s}^{-1}$ apart from the cluster motion center, and it is positioned far from other members of the group (King et al. 2003). Again, this brown dwarf may be part of an an as-yet unrecognized moving group or unassociated.

\section{Additional Sources of Interest}

J0707-4900 (M8.5) is remarkable in its conflicting kinematic, activity and color indicators of age. Originally identified by Ruiz et al. (1991) as a brown dwarf candidate in the Hyades moving group, this source has a large radial velocity $\left(113 \pm 2 \mathrm{~km} \mathrm{~s}^{-1}\right)$ and its $U V W$ velocity components identify it as an intermediate thin/thick disk star (see also Dupuy et al. 2009b). It has the most negative $V$ velocity in our sample, and its prograde Galactic orbit is highly eccentric $(0.46 \pm 0.01)$ but with a modest inclination $(2.5 \pm 1.0)$. However, its spectrum exhibits no obvious signatures of metaldeficiency, and its near-infrared color is unusually red for its spectral type. The weak $\mathrm{H} \alpha$ emission detected in our spectrum $\left(\log _{10} L_{H \alpha} / L_{b o l}=-5.33 \pm 0.06\right)$ is somewhat lower than prior detections $\left(\log _{10} L_{H \alpha} / L_{b o l}=-4.9\right.$ by Tinney \& Reid 1998; $\log _{10} L_{H \alpha} / L_{b o l}=-4.4$ by Mohanty \& Basri 2003), but consistent with a star whose magnetic dynamo has weakened but persists. Mohanty \& Basri (2003) report $v \sin i=10 \mathrm{~km} \mathrm{~s}^{-1}$ for this source, making it a relatively rapid rotator with weak magnetic emission. That study attributes the anomalous behavior of J0707-4900 to a low surface gravity associated with low mass and youth. Hence, the kinematics and low level of activity suggest an old age for J0707-4900, while its color and rapid rotation supports a young age. It is possible that the red color of this source indicates the presence of a mid-to-late L dwarf companion as yet unresolved. The stability in its radial and astrometric motion (three measurements by Ianna \& Fredrick 1995; Tinney 1996 and Henry et al. 2004) argues against the existence of a $\lesssim 0.3$ AU binary, but this source is clearly anomalous and merits further attention. 
J2036+1051 (L3) is one of two unusually active L dwarfs identified in our program. It was first observed by Schmidt et al. (2007) and found to have no $\mathrm{H} \alpha$ emission to a limit of $\mathrm{EW}>-6.3 \AA$. In contrast, we measure $\mathrm{EW}=-11.5 \pm 3.0 \AA$ ( $\mathrm{H} \beta$ and $\mathrm{H} \gamma$ lines were too weak to be detected). It appears that we caught this rapidly rotating $\operatorname{dwarf}\left(v \sin i=67.1 \pm 1.5 \mathrm{~km} \mathrm{~s}^{-1}\right.$; Blake et al. 2010) in a flare state. We note that the alkali lines in this source are a little weak compared to other L3 dwarfs in the sample (with the exception of J0823-4912AB), but the kinematics are consistent with an older disk source. The alkali lines may have been filled in by continuum emission from the flare.

J2037-1137 (M8) is the earliest-type source in our sample to exhibit no detectable $\mathrm{H} \alpha$ emission, to an EW limit of $>-0.3 \AA$ and $\log _{10} L_{H \alpha} / L_{b o l}<-6.74$. In contrast, prior studies have consistently detected emission in the range $-5.51 \lesssim \log _{10} L_{H \alpha} / L_{b o l} \lesssim-4.48$ (Schmidt et al. 2007; Reiners \& Basri 2010; Lee et al. 2010). Unlike J2036+1051, this slowly rotating dwarf $(v \sin i \leq$ $3 \pm 2 \mathrm{~km} \mathrm{~s}^{-1}$; Reiners \& Basri 2009) may have been observed during a minimum in its magnetic emission cycle, or in an orientation exhibiting a relatively quiescent surface.

\section{Summary}

This paper has reported the radial velocities of 85 VLM stars and brown dwarfs of spectral types M6-L6 with MagE with typical precisions of $2-3 \mathrm{~km} \mathrm{~s}^{-1}$. Combining these with previously published proper motions and distances, we computed $U V W$ velocities and examined velocity dispersions as a function of spectral type, color and magnetic activity. We find that unusually blue objects are more dispersed than unusually red objects, in support of color effects being driven by higher surface gravities and/or slightly subsolar metallicities among an older, low-mass stellar population. We also find that magnetically inactive L dwarfs are more dispersed than magnetically active L dwarfs, following the age-activity relations of earlier-type stars. The most interesting finding is the greater dispersions and $U$ velocity offset (net radial flow) of $\mathrm{L}$ dwarfs as compared to late-M dwarfs. The greater dispersions of L dwarfs affirms prior results by Seifahrt et al. (2010) and Blake et al. (2010), but we speculate that this may be driven by a large fraction of unusually blue L dwarfs in local $20 \mathrm{pc}$ sample as compared to deeper imaging surveys. Population simulations incorporating brown dwarf evolution, Galactic dynamics in an axisymmetric potential, and local selection still predict that L dwarfs should be younger than late-M dwarfs, although this can be reversed if brown dwarfs have had a different formation history than stars (or alternately, evolve more quickly than evolutionary models predict). However, these simulations cannot reproduce the distinct $U$ velocity distribution of L dwarfs, which is either driven by non-axisymmetric Galactic structure (e.g., bar, spiral arms) or the existence of a distinct, dispersed population of L dwarfs in the Solar Neighborhood. We also identify 8 kinematic members of nearby YMGs, including new candidates J0823-4912AB in the $\beta$ Pictoris Moving Group (although likely part of an older association/stream) and J1510-2818 in the Argus Association. Three additional sources, J0652-2534, J0909-0658 and J1411-2119 have

evidence of low surface gravities but no YMG assignment; these may be members of older moving 
groups or streams, or simply solivagant $\sim 500$ Myr-old brown dwarfs.

The ultimate goal of the Brown Dwarf Kinematics Project is a complete kinematic sampling of the lowest-mass stars and brown dwarfs in the vicinity of the Sun. With $25 \%$ of the 20 pc late-M and L dwarf sample studied here, we are likely still missing additional members of nearby YMGs, and we may still be subject to (unknown) selection biases due to incompleteness. Work is underway to complete the RV sampling of the local VLM population. Given preliminary evidence of distinct kinematic populations of L dwarfs in the Solar Neighborhood, examining the kinematics of $\mathrm{T}$ dwarfs (all of which are substellar) may provide an important anchor for disentangling the mass function and star formation history of our local mixed stellar/substellar population.

\section{Acknowledgements}

The authors would like to thank Jorge Araya, Mauricio Martinez, Hernan Nunez, and Geraldo Vallardes for assistance in the observations. We acknowledge useful discussions with John Gizis during the preparation of the manuscript. A.A.W acknowledges funding from NSF grants AST1109273 and AST-1255568 and support from the Research Corporation for Science Advancement?s Cottrell Scholarship; E.E.M acknowledges support from NSF grant AST-1313029. This publication makes use of data products from the Two Micron All Sky Survey, which is a joint project of the University of Massachusetts and the Infrared Processing and Analysis Center/California Institute of Technology, funded by the National Aeronautics and Space Administration and the National Science Foundation. This research has benefitted from the M, L, and T dwarf compendium housed at DwarfArchives.org and maintained by Chris Gelino, Davy Kirkpatrick, and Adam Burgasser. It has also made use of the SIMBAD database and VizieR service, operated at CDS, Strasbourg, France. This article has also made use of data from the Sloan Digital Sky Survey. Funding for the SDSS and SDSS-II has been provided by the Alfred P. Sloan Foundation, the Participating Institutions, the National Science Foundation, the U.S. Department of Energy, the National Aeronautics and Space Administration, the Japanese Monbukagakusho, the Max Planck Society, and the Higher Education Funding Council for England. The SDSS Web Site is http://www.sdss.org/. The SDSS is managed by the Astrophysical Research Consortium for the Participating Institutions. The Participating Institutions are the American Museum of Natural History, Astrophysical Institute Potsdam, University of Basel, University of Cambridge, Case Western Reserve University, University of Chicago, Drexel University, Fermilab, the Institute for Advanced Study, the Japan Participation Group, Johns Hopkins University, the Joint Institute for Nuclear Astrophysics, the Kavli Institute for Particle Astrophysics and Cosmology, the Korean Scientist Group, the Chinese Academy of Sciences (LAMOST), Los Alamos National Laboratory, the Max-Planck-Institute for Astronomy (MPIA), the Max-Planck-Institute for Astrophysics (MPA), New Mexico State University, Ohio State Uni-

versity, University of Pittsburgh, University of Portsmouth, Princeton University, the United States Naval Observatory, and the University of Washington.

Facilities: Magellan:Clay 


\section{A. Conversion between 2MASS and MKO $J H K_{s}$ Magnitudes}

To compute the bolometric fluxes for our sources, we made use of their 2MASS $J$-band photometry and MKO J-band bolometric corrections from Liu et al. (2010). This required a small correction between filter magnitude systems due to the highly structured spectra of late $\mathrm{M}$ and $\mathrm{L}$ dwarfs. Stephens \& Leggett (2004) have previously reported corrections for L and T dwarfs as a funciton of spectral type, but did not include late-M dwarfs in their sample; we therefore computed a new conversion relation spanning types M6 to L7. We selected 533 low-resolution, near-infrared spectra form the SpeX Prism Library (SPL; Burgasser 2014) with reported optical classifications between M6 and L7 and median S/N > 100. We computed spectrophotometric magnitudes in both 2MASS and MKO $\mathrm{JHK}_{s}$ systems following the procedures described in Stephens \& Leggett (2004) and Cushing et al. (2005). Figure 20 displays the magnitude differences ( $\Delta=$ MKO-2MASS) in $J$, $H$ and $K_{s}$ as a function of spectral type. We fit all three filter differences to second-order polynomials (higher orders did not significantly improve the fits), iteratively rejecting $3 \sigma$ outliers. The polynomial coefficients and dispersions are listed in Table 11, Both $J$ and $H$ show significant (and opposing) trends, and the polynomial fits produce a residual scatter of 0.005-0.008 mag. 


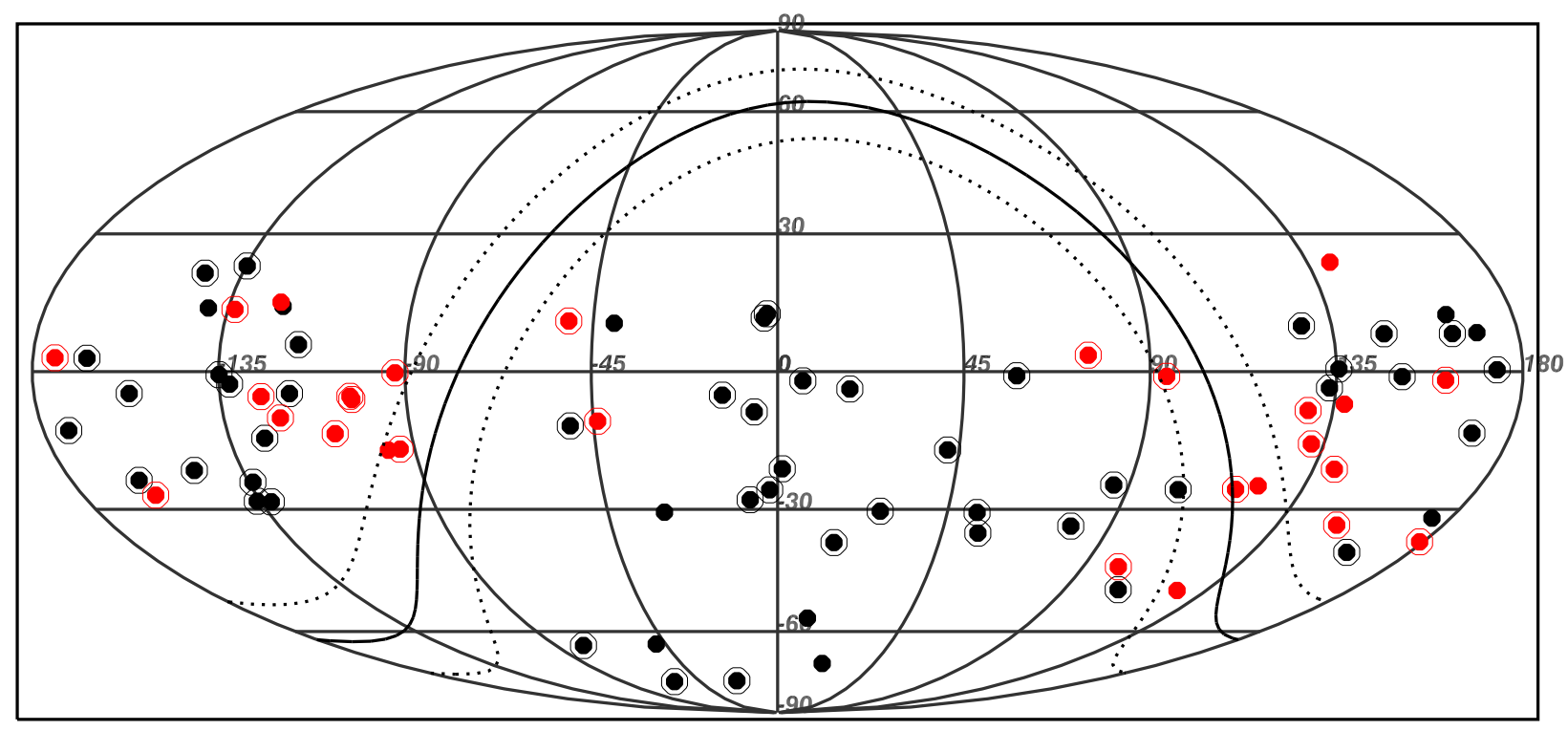

Fig. 1. - Mollweide projection equatorial map of late-M dwarfs (black) and L dwarfs (red) in our sample. Sources within $20 \mathrm{pc}$ are encircled. The Galactic plane and $\pm 10^{\circ}$ about the plane are indicated by the solid and dotted lines, respectively. 

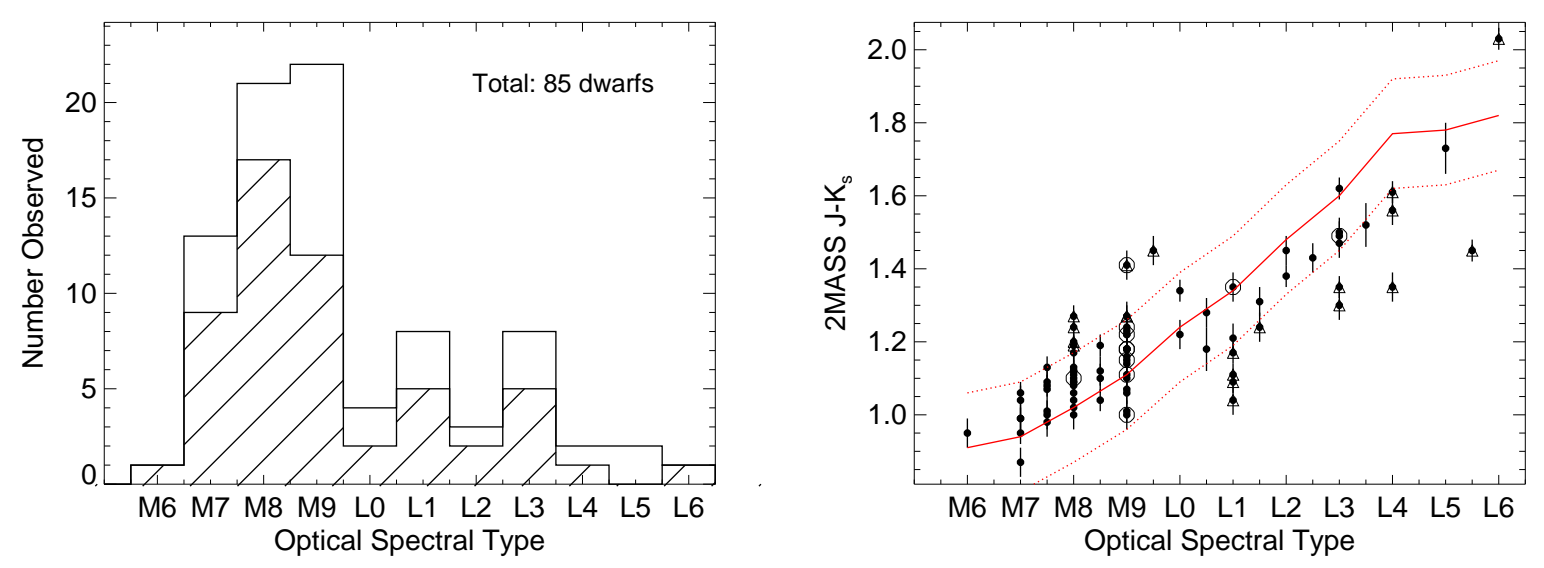

Fig. 2.- (Left): Distribution of optical spectral types for the 85 observed sources, sampled by whole subtype bins. The open histogram refers to all sources observed; the hatched histogram refers to those sources with previously published radial velocities. (Right): 2MASS $J-K_{S}$ colors of our targets compared to the mean colors of M6-L6 dwarfs (red line) from Schmidt et al. (2010) and West et al. (2011). Our \pm 0.15 mag threshold for unusually red and blue dwarfs are indicated by dotted lines, and those sources are highlighted by open triangles. Young sources in our sample are highlighted with open circles. 


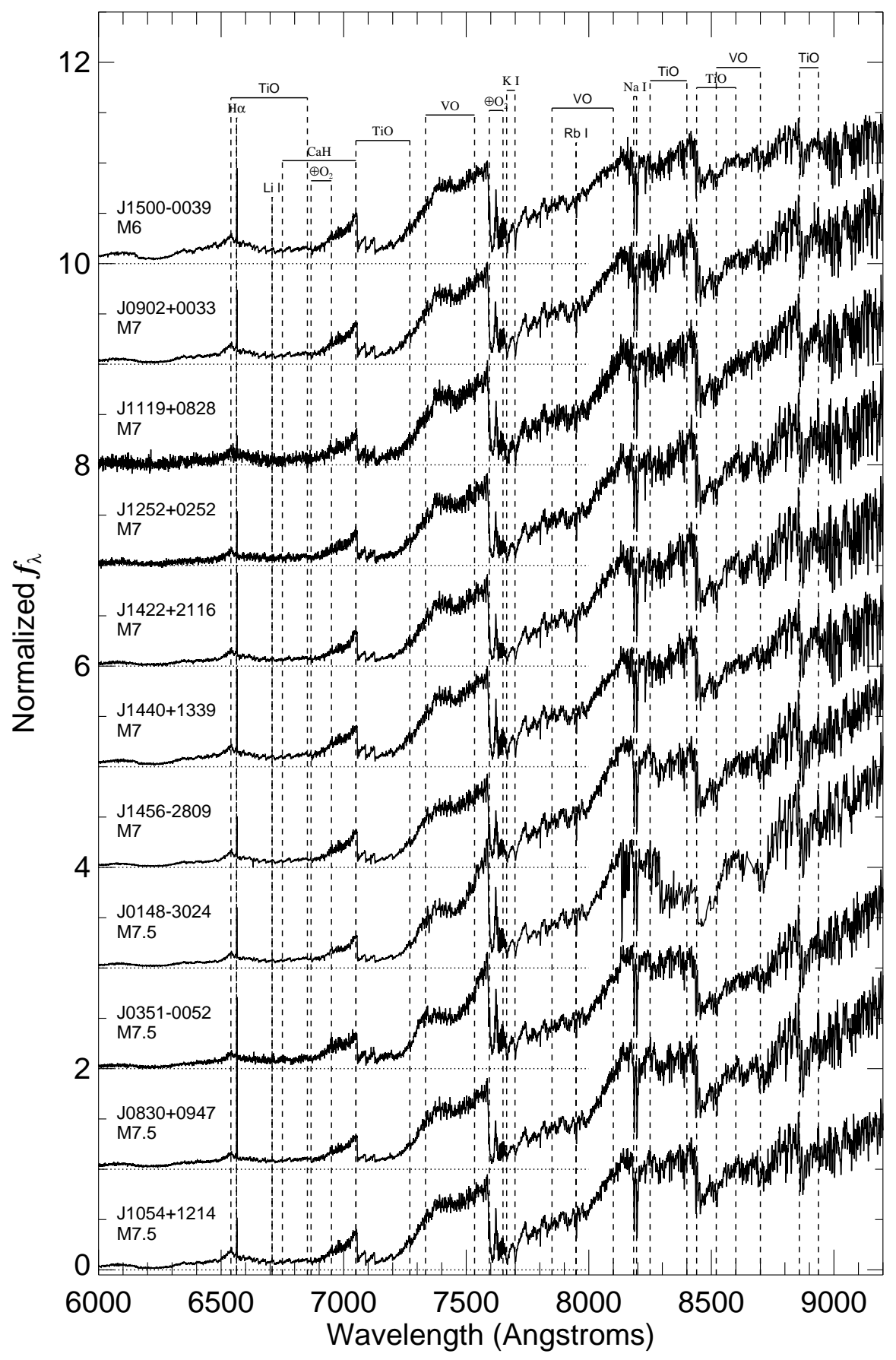

Fig. 3.- Observed MagE spectra of our sample, ordered by spectral type and right ascension. Data are normalized in the $8200-8600 \AA$ range and each spectrum offset by a constant for clarity. Major spectral features from Li I, K I, Na I, Rb I, Cs I, TiO, VO, CrH, FeH, CaH and H I emission are labeled, as is telluric $\mathrm{O}_{2}$ absorption. 


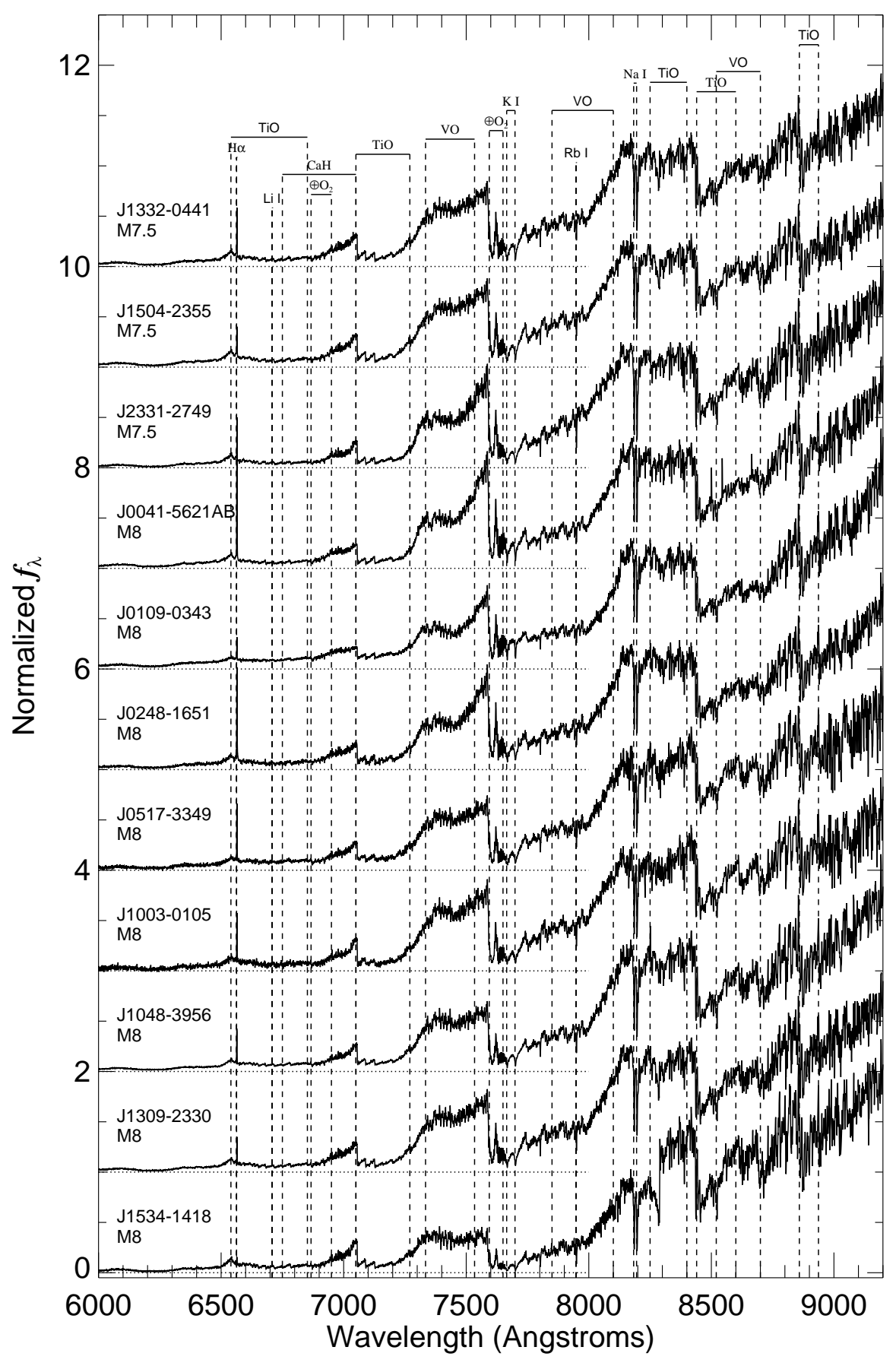

Fig. 3.- Cont. 


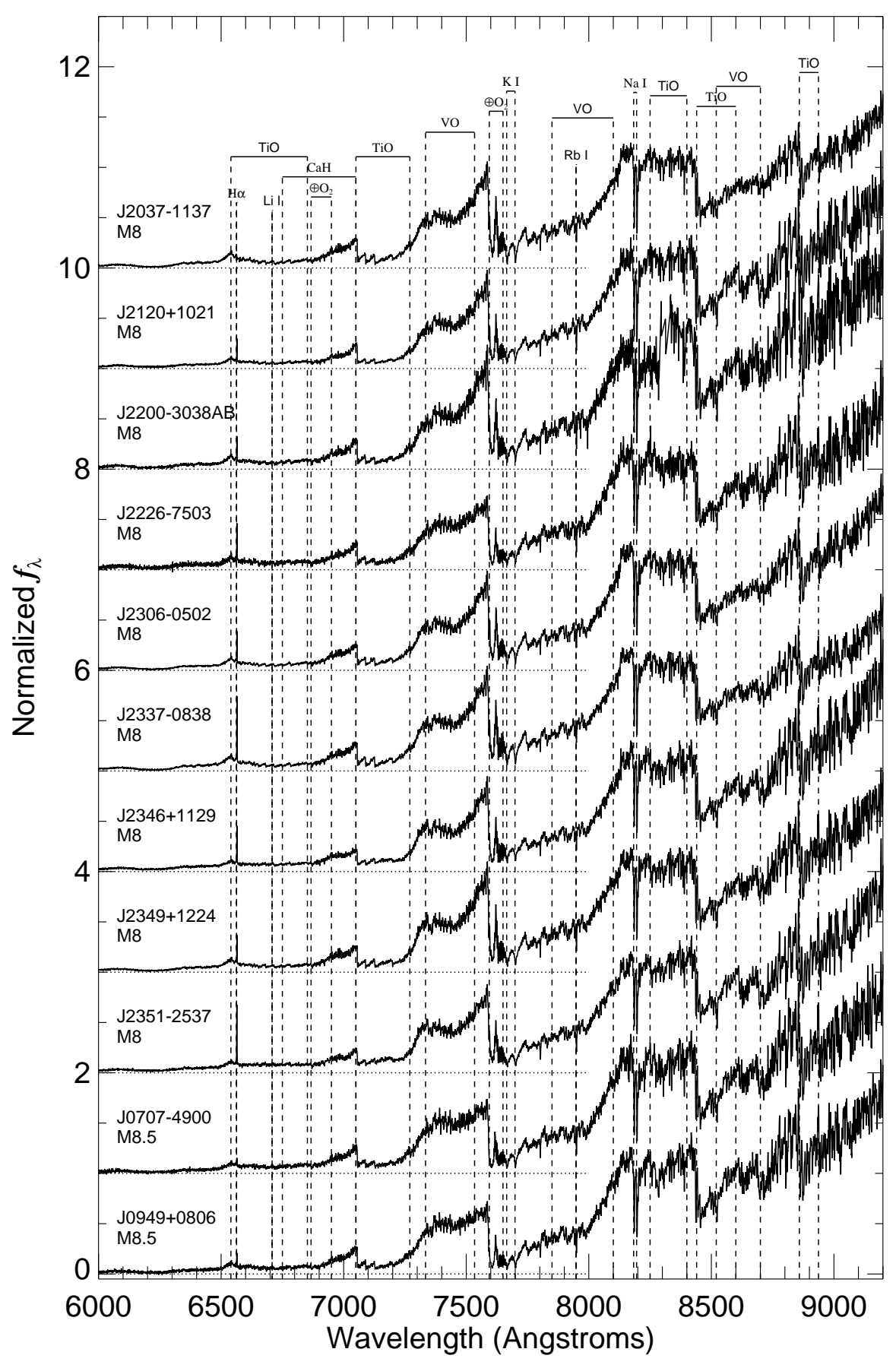

Fig. 3.- Cont. 


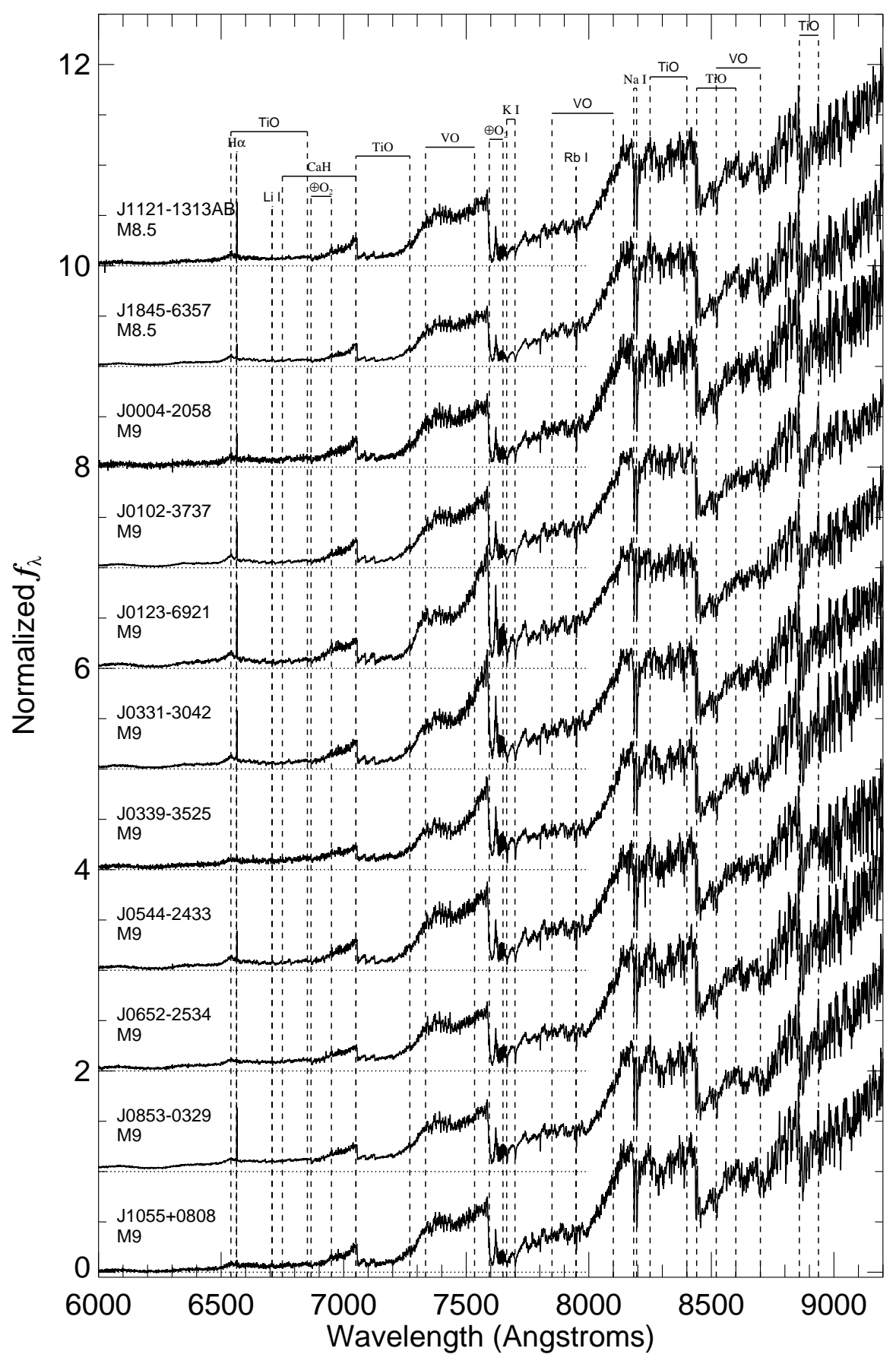

Fig. 3.- Cont. 


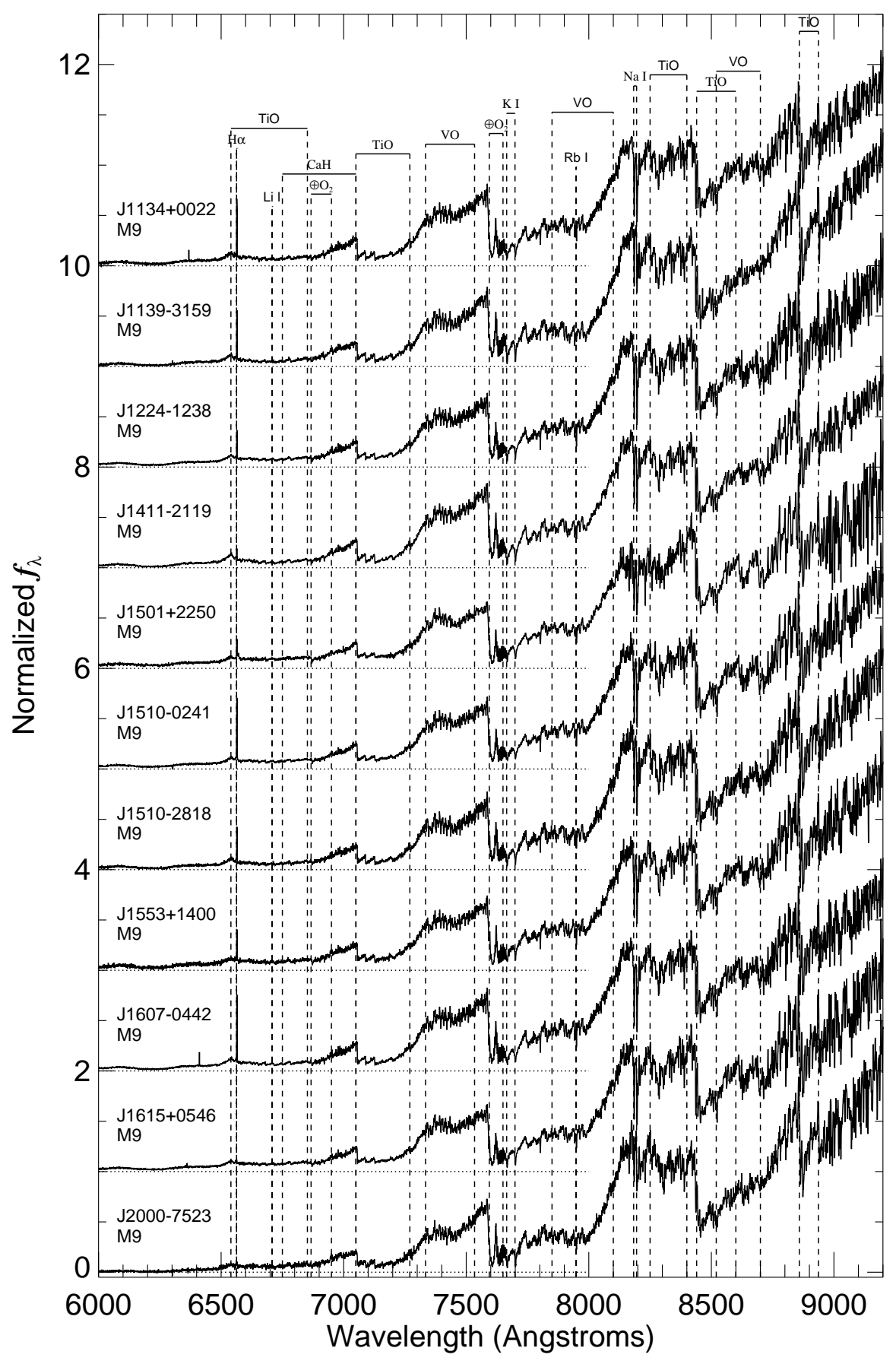

Fig. 3.- Cont. 


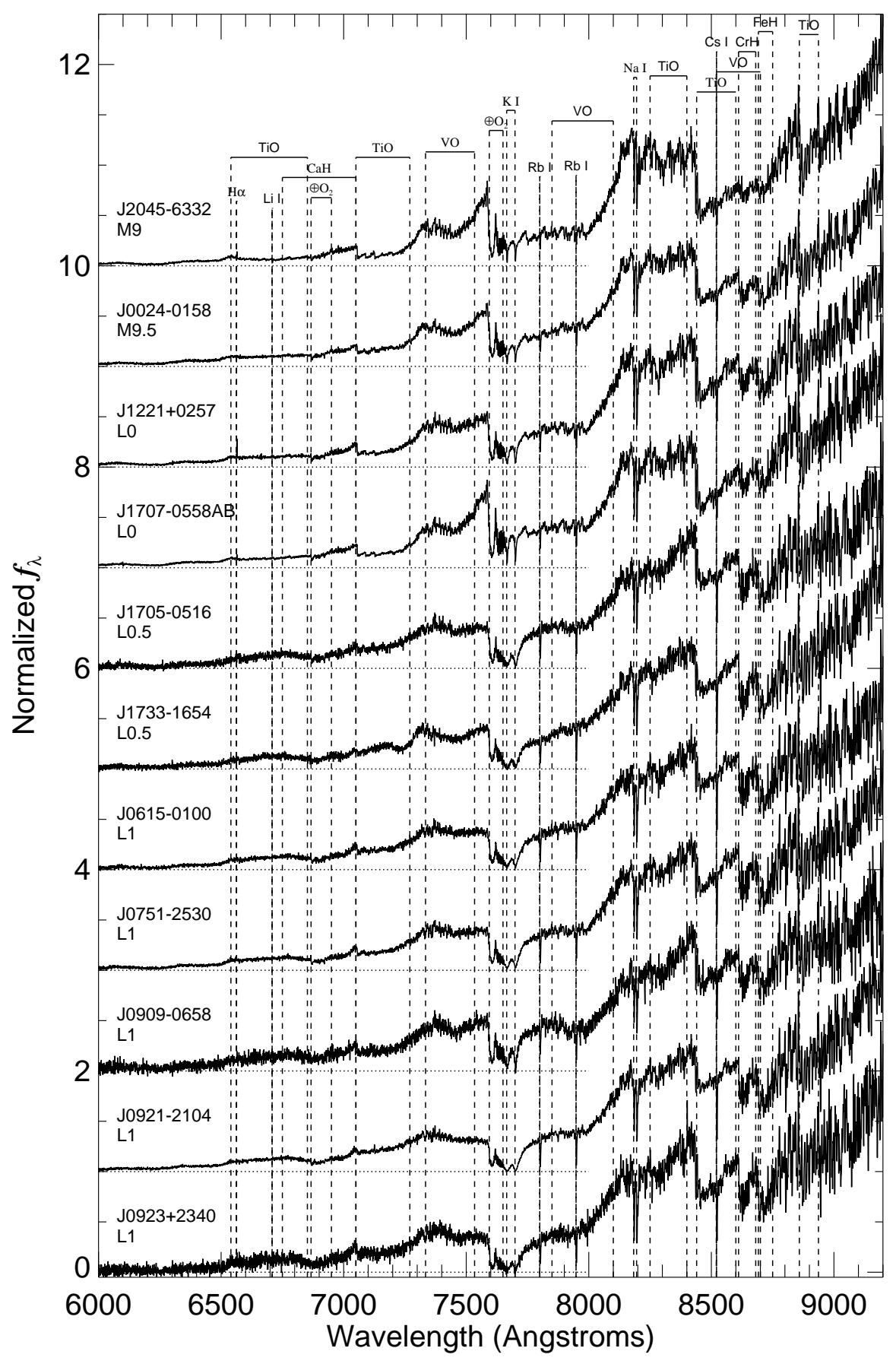

Fig. 3.- Cont. 


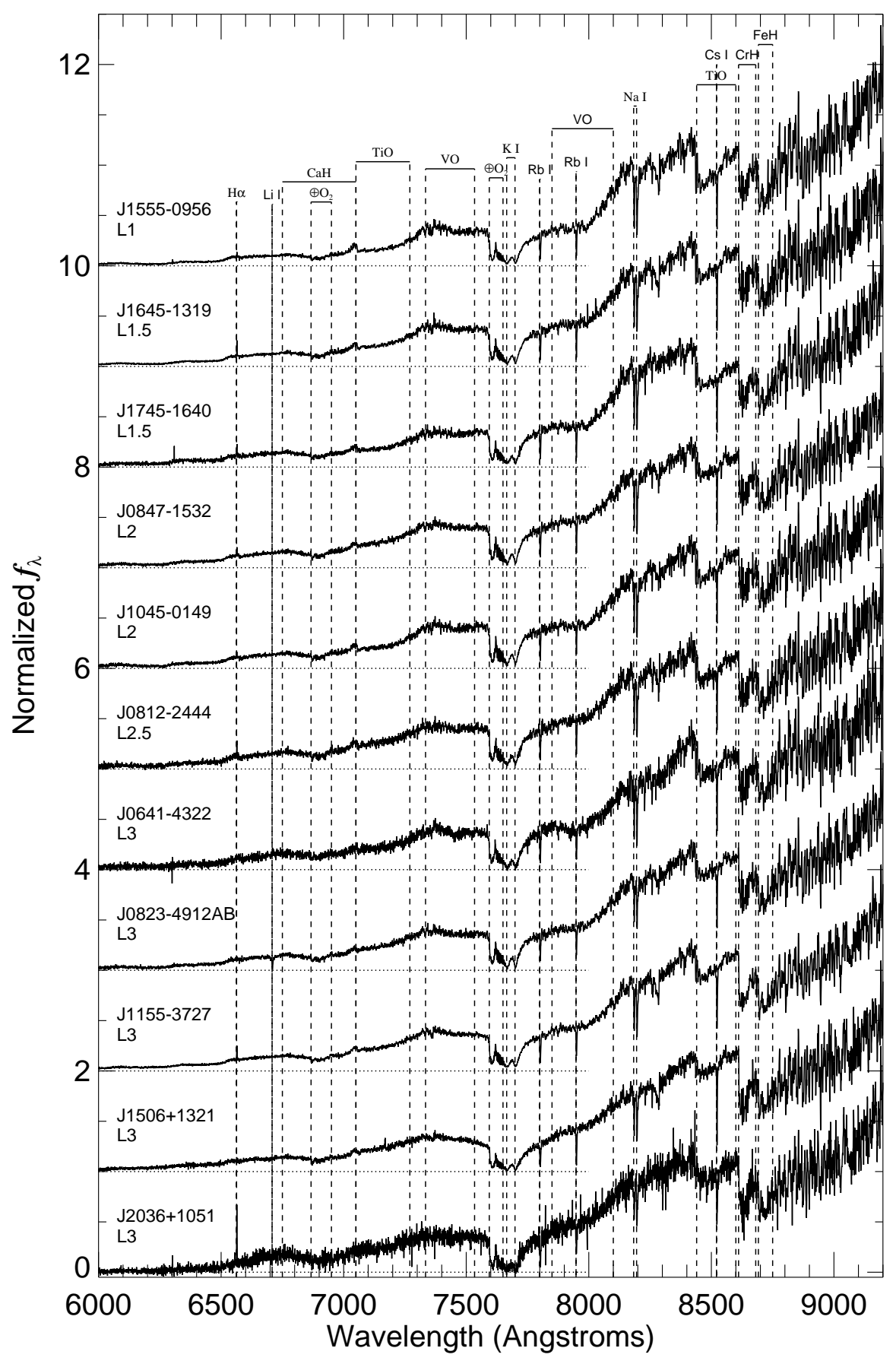

Fig. 3.- Cont. 


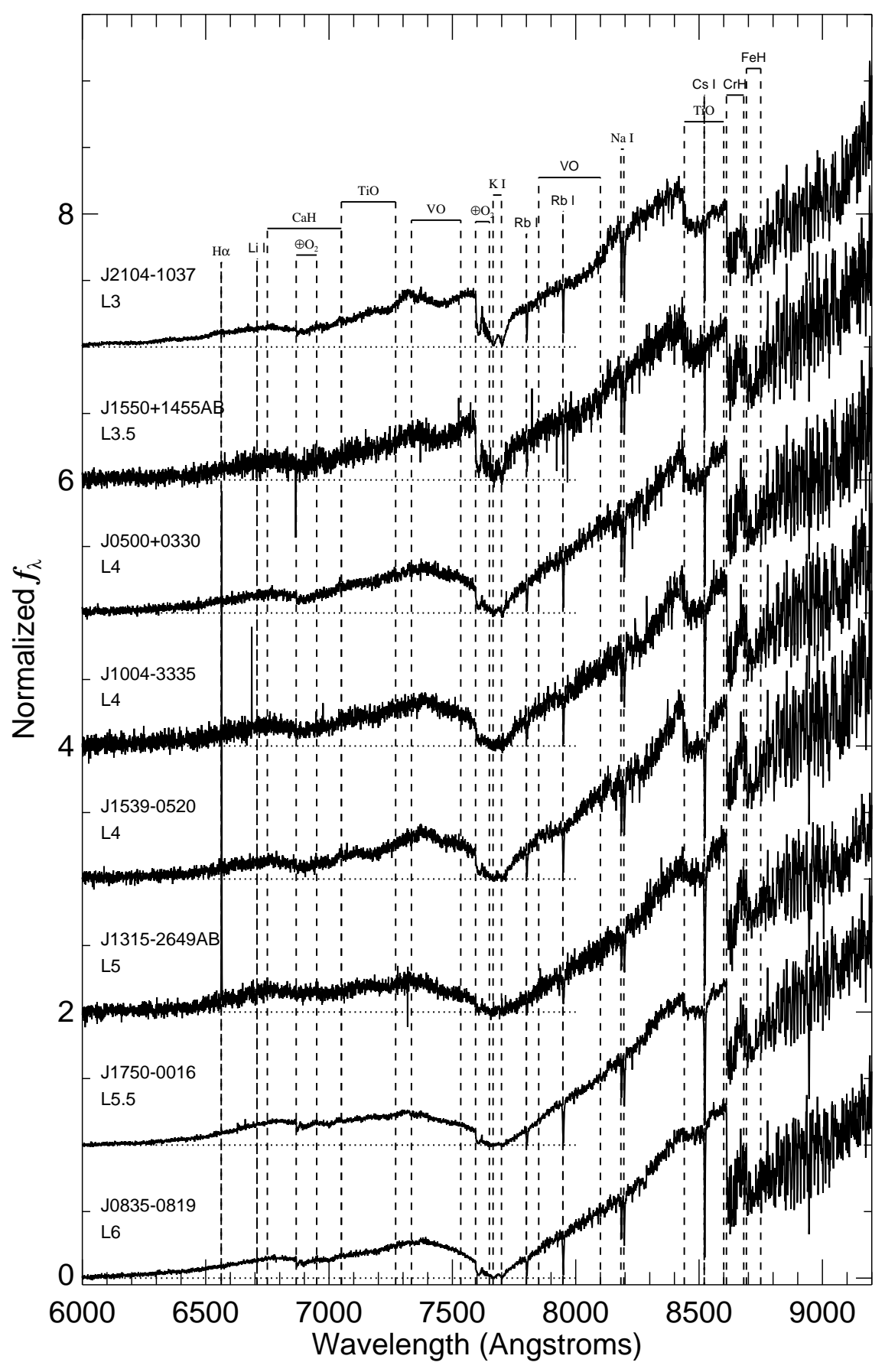

Fig. 3.- Cont. 

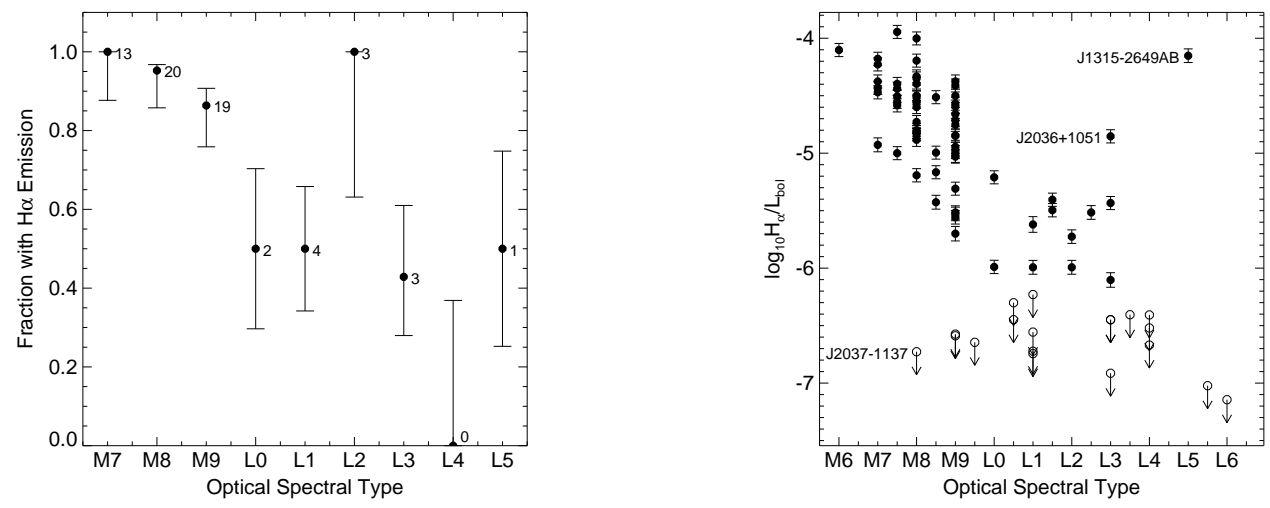

Fig. 4.- (Left): Fraction of sources in our sample exhibiting $\mathrm{H} \alpha$ emission as a function of spectral type. Uncertainties are based on binomial statistics, with the numbers next to each point indicating the total number of objects exhibiting $\mathrm{H} \alpha$ in that subtype bin. (Right): $\log _{10} L_{H \alpha} / L_{b o l}$ as a function of spectral type; upper limits are indicated by downward arrows. The two unusually active L dwarfs J2036+1051 and J1315-2649 are labeled, as is the unusually inactive M8 dwarf J2037-1137.
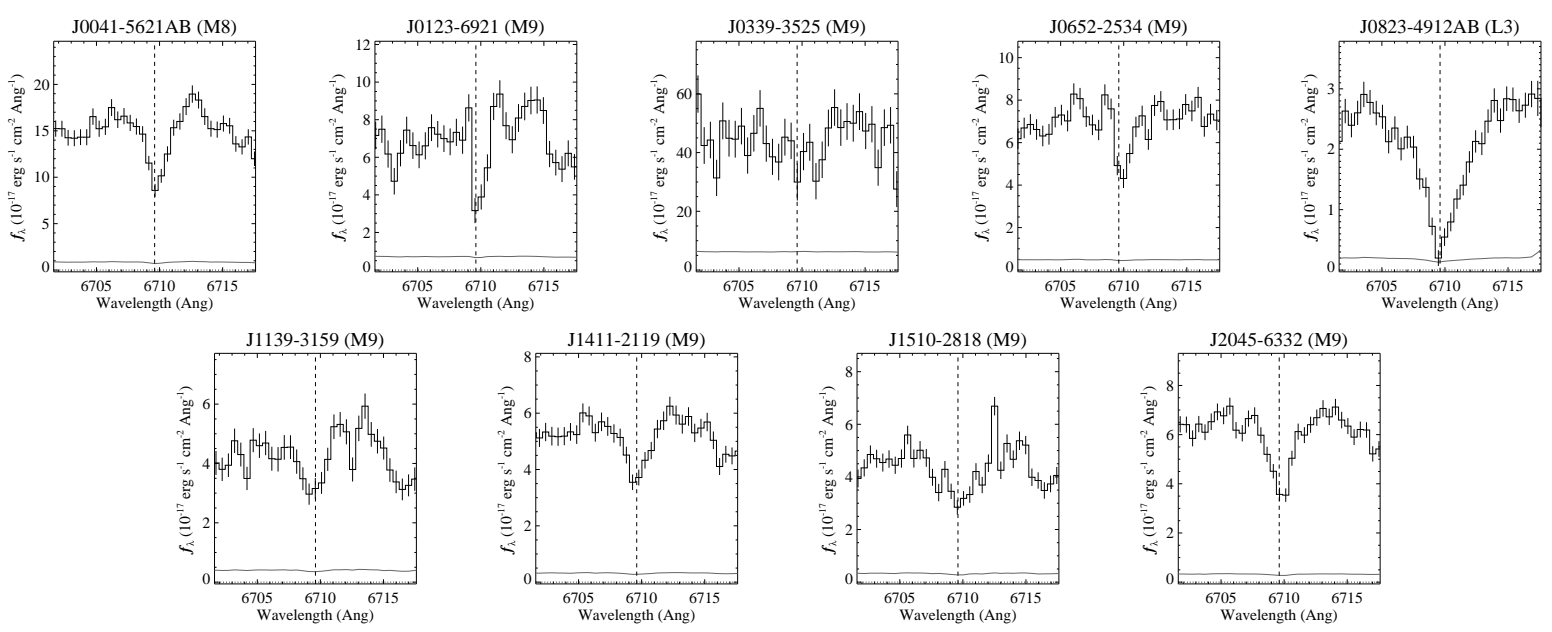

Fig. 5.- 6700-6720 $\AA$ spectra of nine sources in our sample that show significant or marginal absorption from the $6710 \AA$ Li I line, indicating that they are young brown dwarfs. 

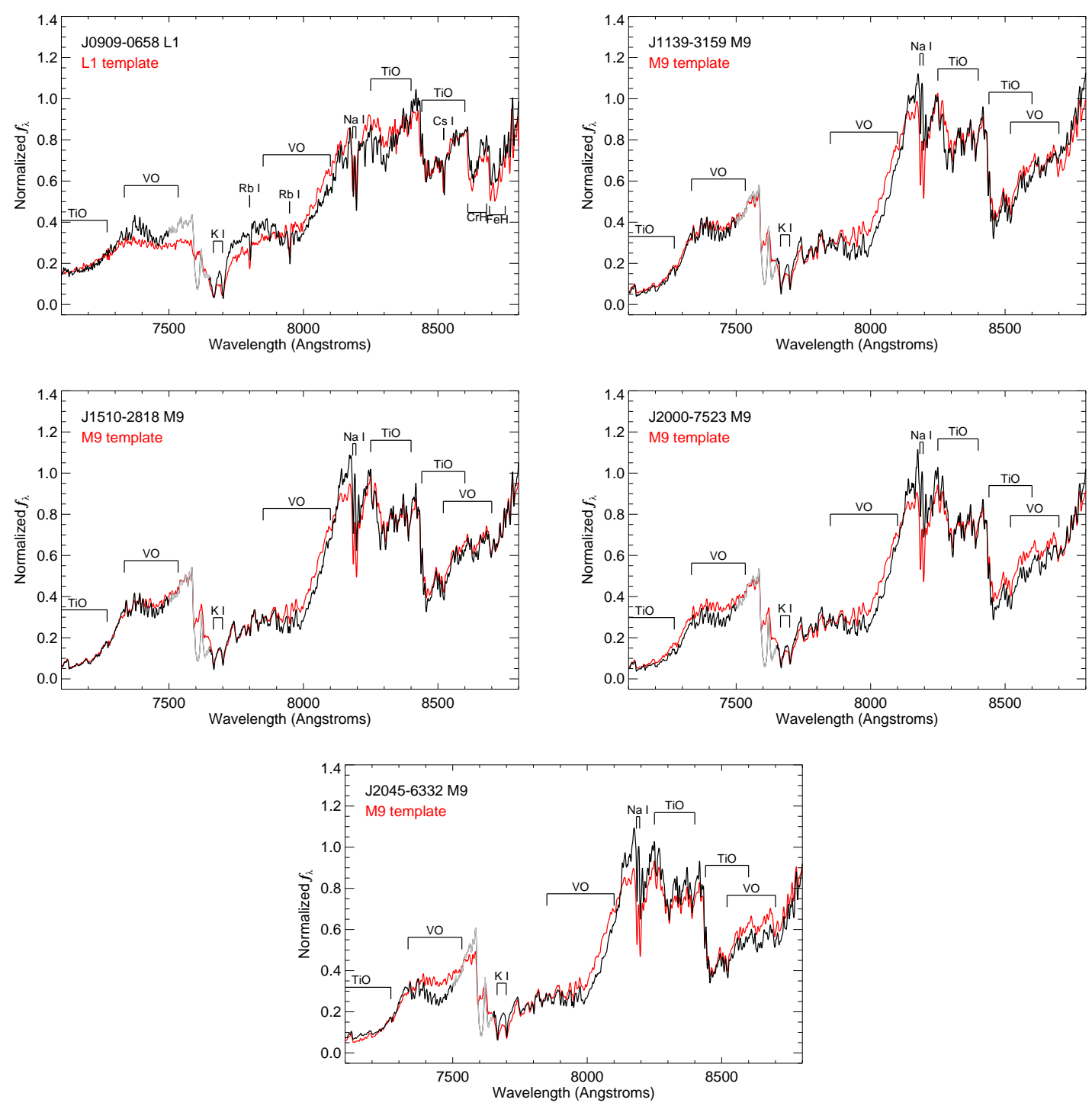

Fig. 6. - 7100-8800 A spectra of five sources (black) exhibiting strong features of low surface gravity, compared to equivalently-classified SDSS templates (red). Spectra are normalized at $8200 \AA$; the 7500-7600 $\AA$ region affected by poor flux calibration is indicated in gray and should be ignored. 

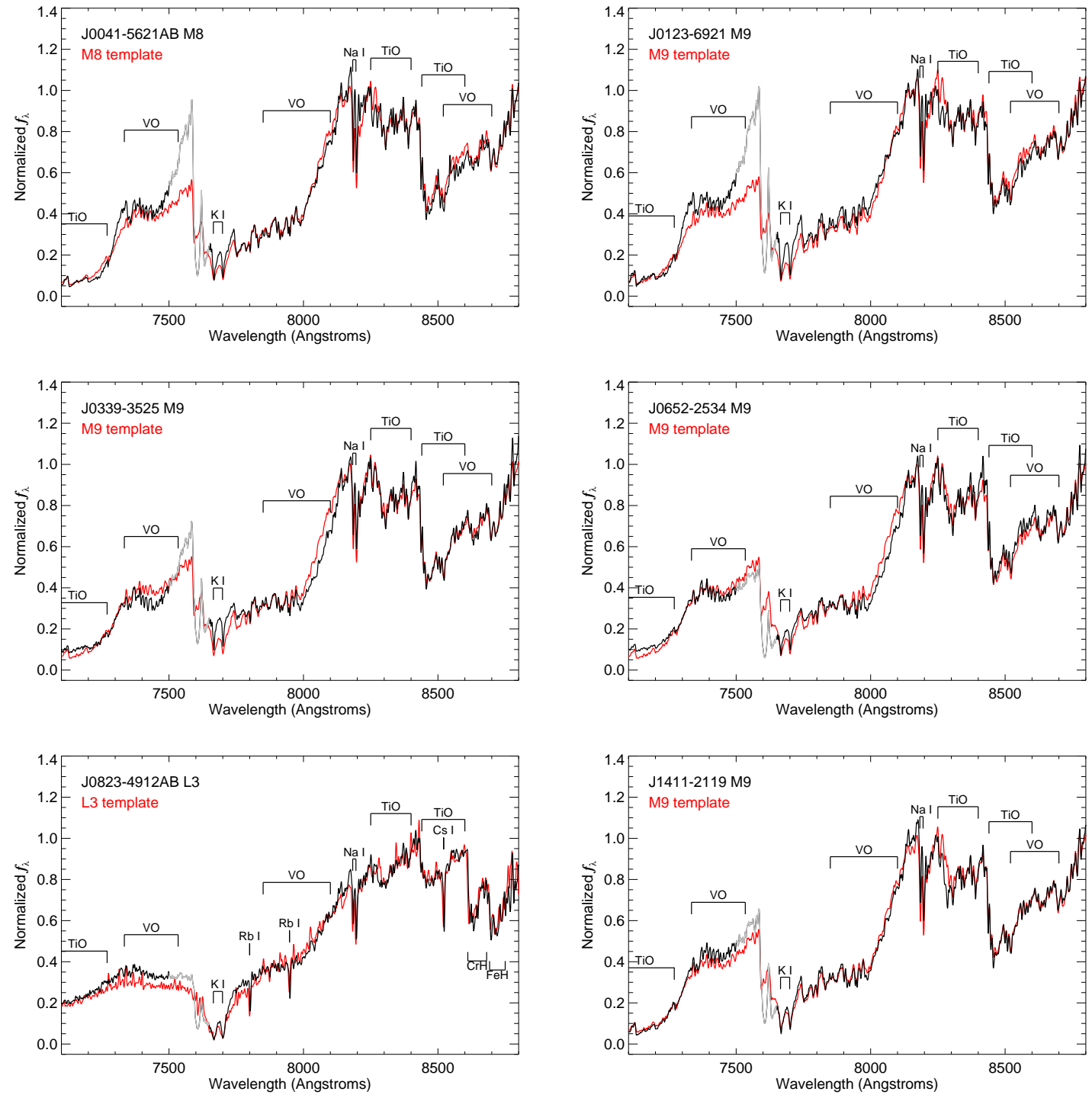

Fig. 7.- Same as Figure 6, showing sources exhibiting weak signatures of low surface gravity. 

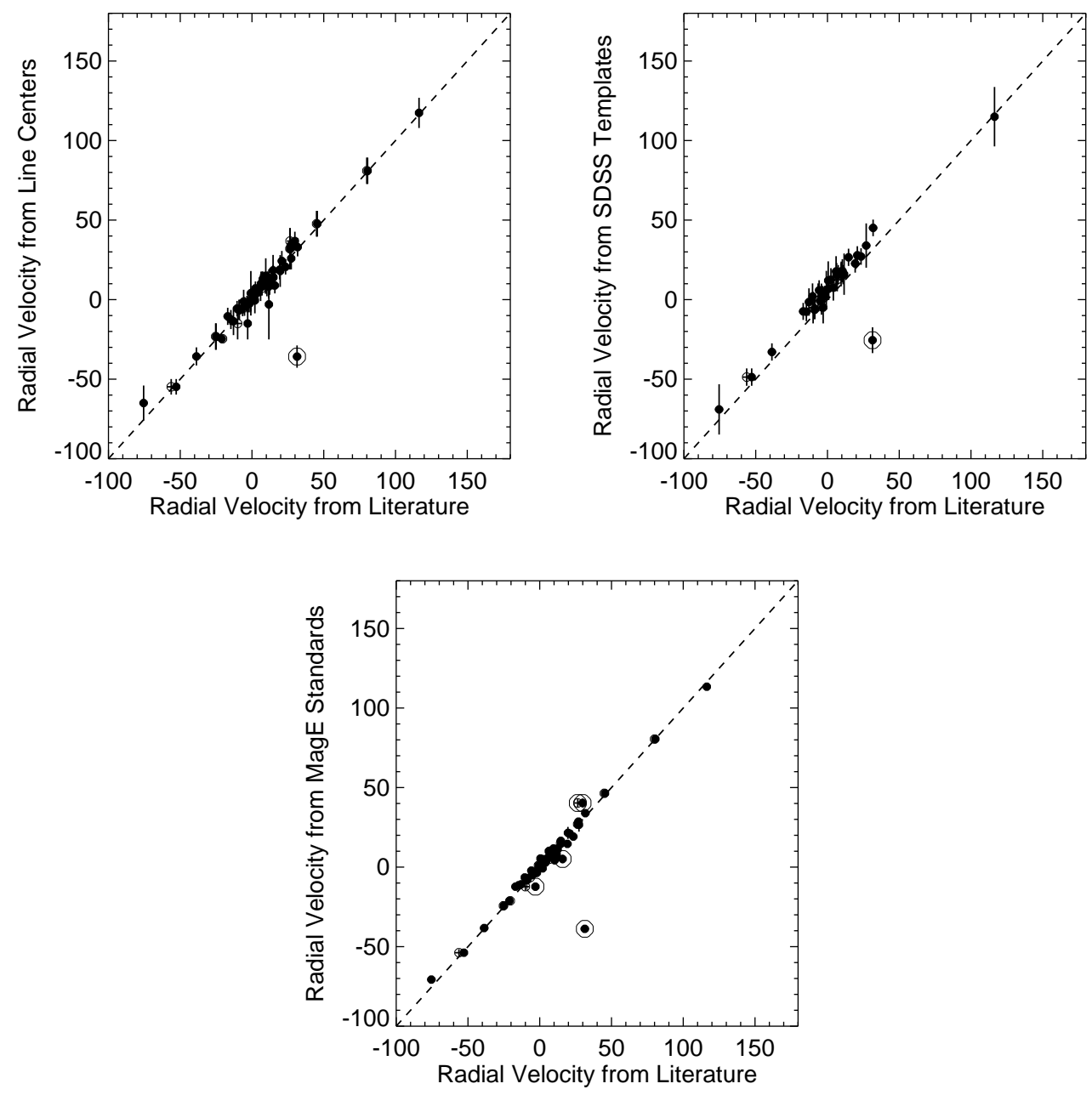

Fig. 8.- Comparison of RVs measured from line centers (upper left panel), cross-correlation with SDSS templates (upper right panel) and cross-correlation with MagE RV standards (lower panel) to previously published high precision measurements ( $\leq 3 \mathrm{~km} \mathrm{~s}^{-1}$; Table1). The consistent deviant, J0517-3349, is highlighted by an open circle; this appears to be a sign reversal in the RV reported by Reiners \& Basri (2008). The other deviants in the lower panel, J0500+0330, J0835+0819 and J2351-2537, are discussed further in Section 3.4. 

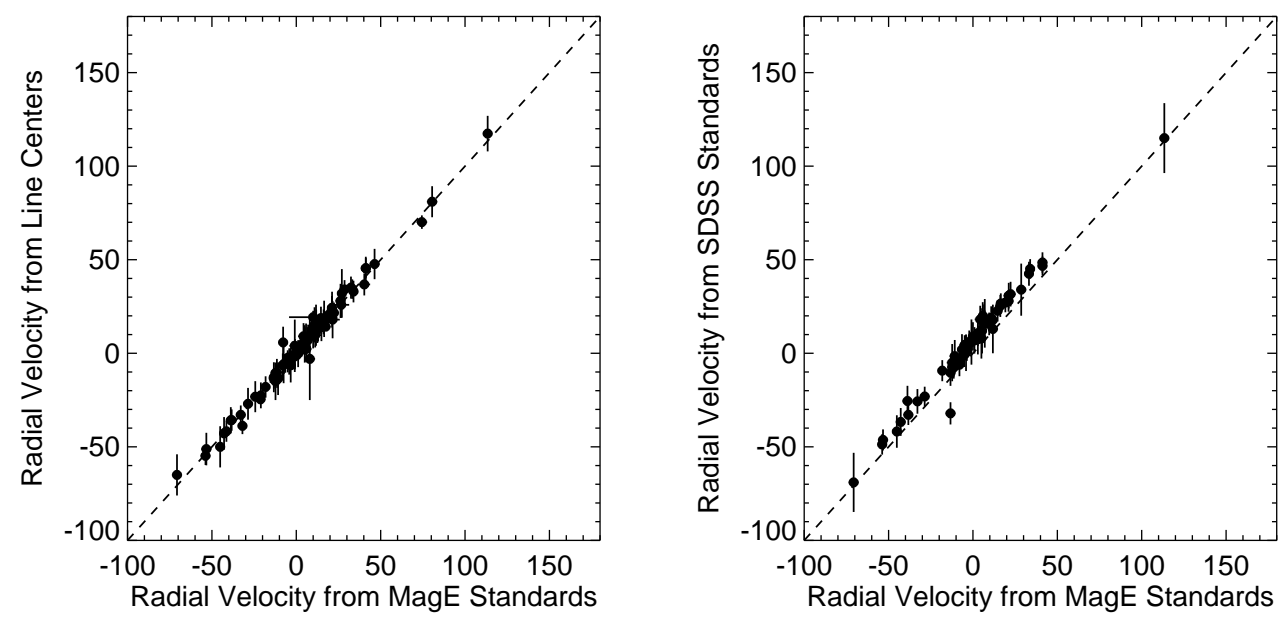

Fig. 9.- Comparison of RV measurements between line centers, SDSS templates, and MagE RV standards. 

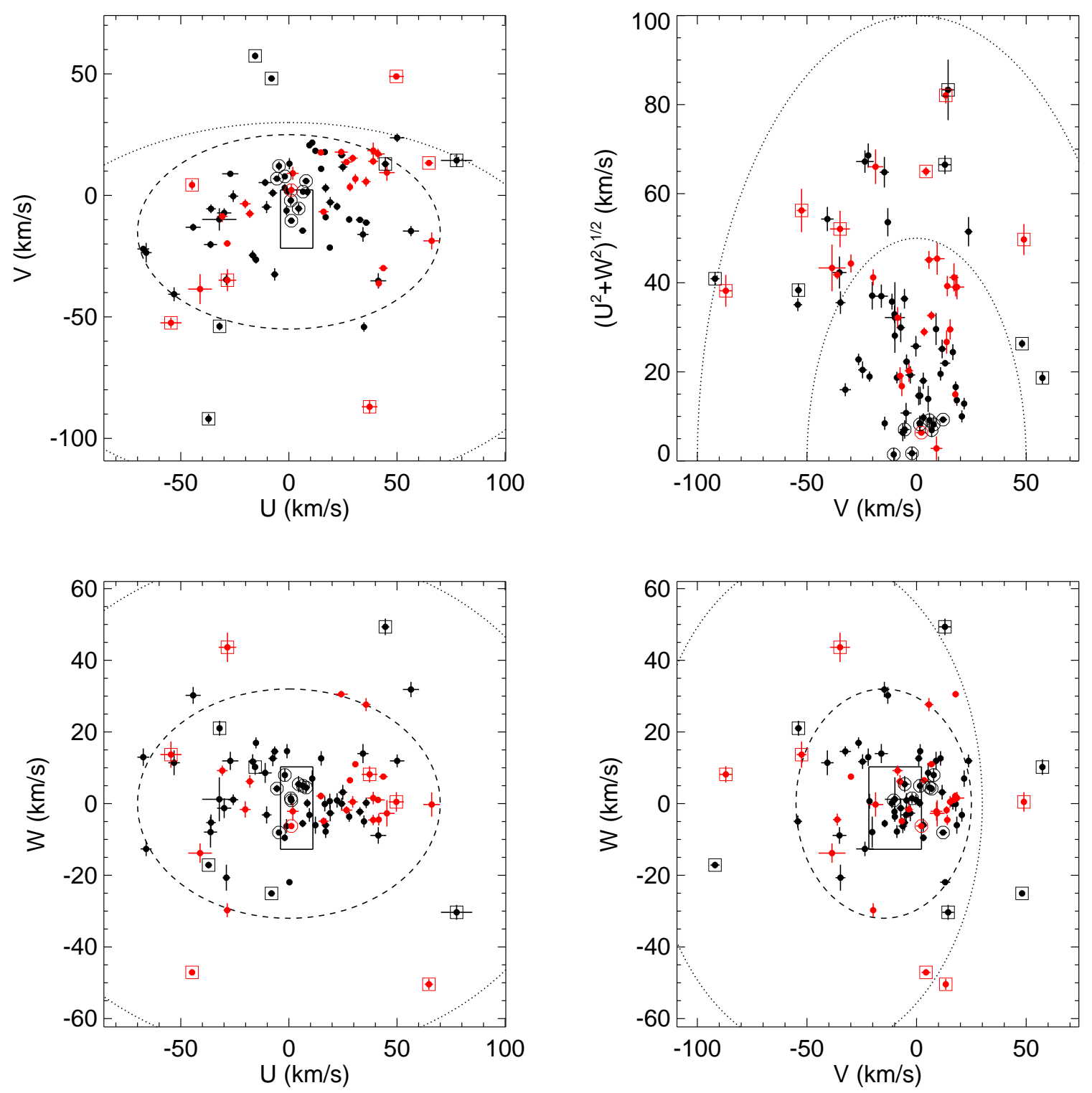

Fig. 10.- $U V W$ space motions for our sample in the Local Standard of Rest. Upper left, lower left and lower right plots compare velocity components to the $2 \sigma$ velocity means and dispersions of Galactic thin disk (dashed lines) and thick disk (dotted lines) populations from Bensby et al. (2003). Also shown is the "good box" of Zuckerman \& Song (2004), a rough locus of nearby YMG members. The upper right panel shows a Toomre plot, with dotted lines delineating $50 \mathrm{~km} \mathrm{~s}^{-1}$ steps of constant $v_{t o t}=\left(U^{2}+V^{2}+W^{2}\right)^{1 / 2}$. Late-M dwarfs are indicated by black symbols, $\mathrm{L}$ dwarfs by red symbols. Sources identified as intermediate thin/thick disk stars are highlighted by open squares; sources exhibiting Li I absorption are highlighted by open circles. 

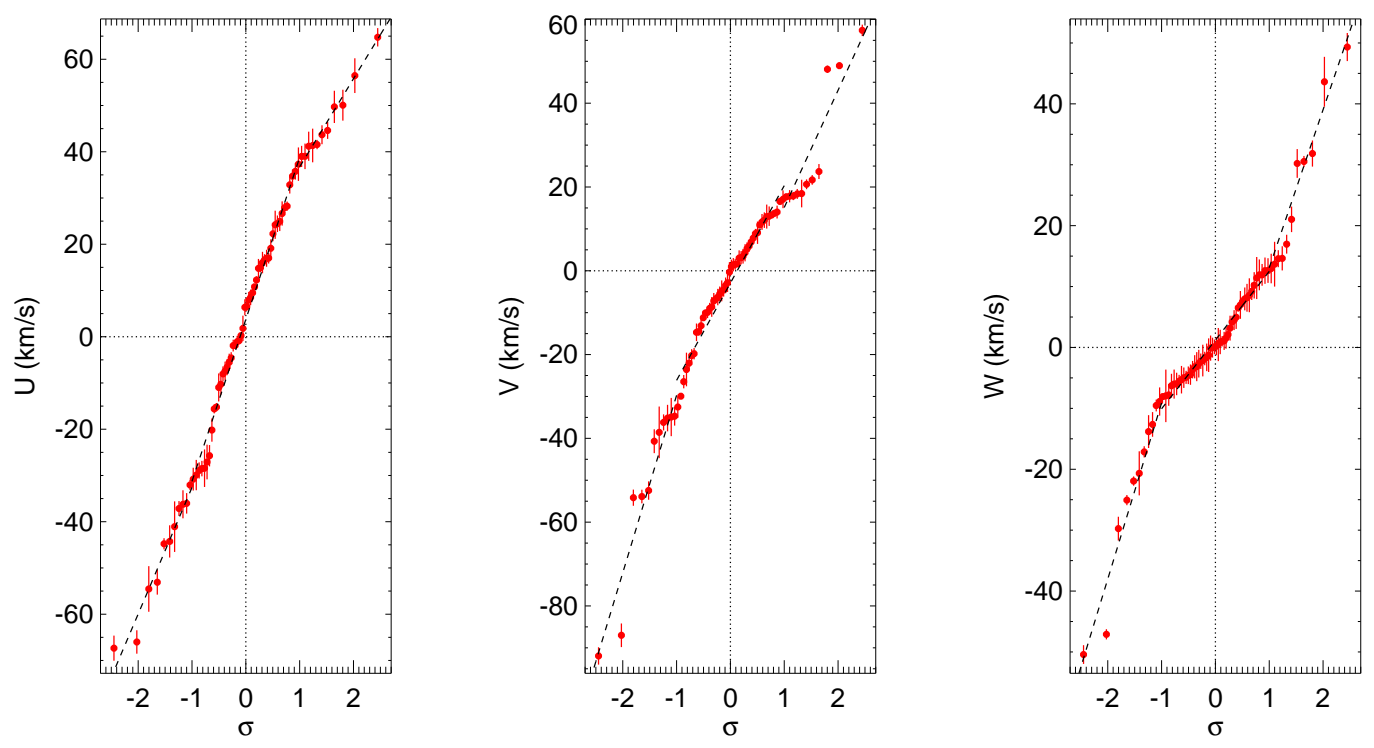

Fig. 11.- Probit plots for $U, V$, and $W$ velocity components for sources in our sample within 20 pc of the Sun. Individual velocity measurements and uncertainties are indicated in red. Separate linear fits are shown for core $(|\sigma|<1)$ and warm $(|\sigma|>1)$ populations. Dotted lines mark the mean of the velocity distributions $(\sigma=0)$ and zero velocity.
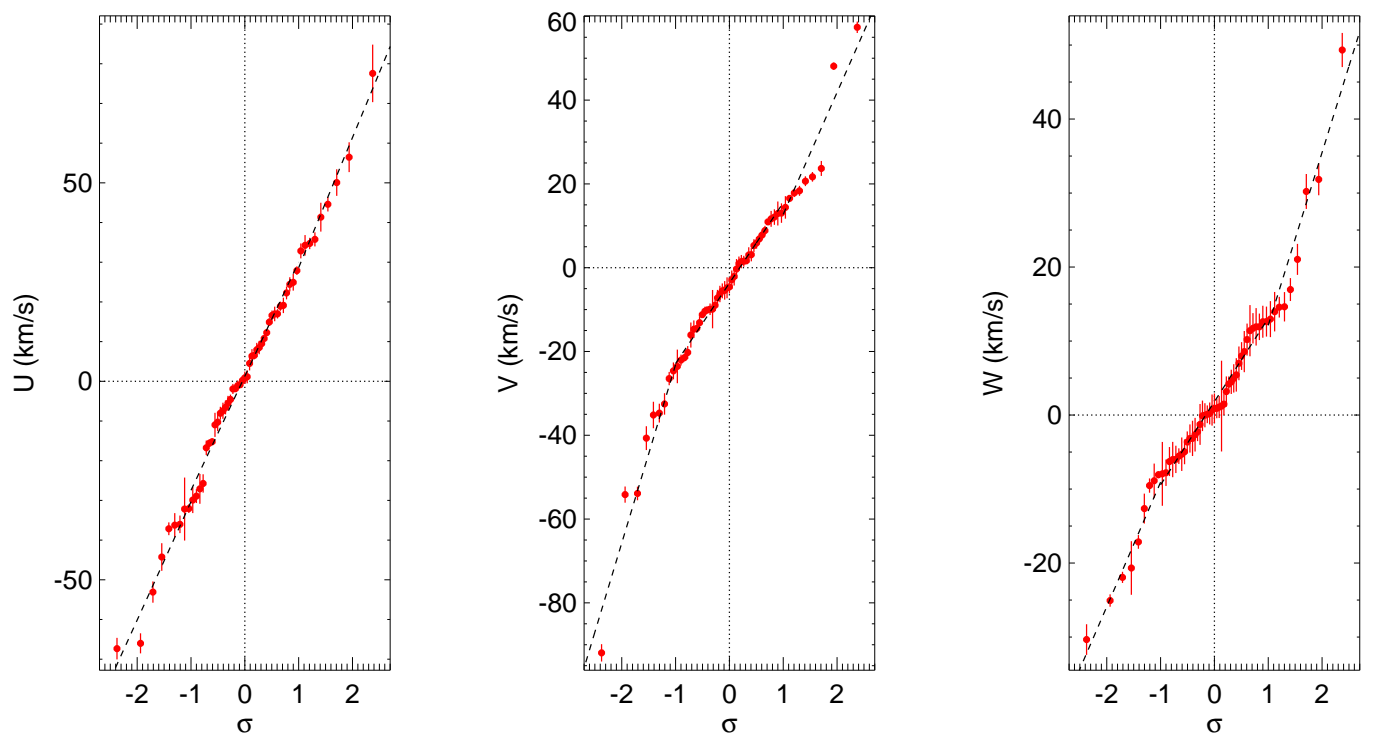

Fig. 12.- Same as Figure 11 for the late-M dwarfs in our sample. 

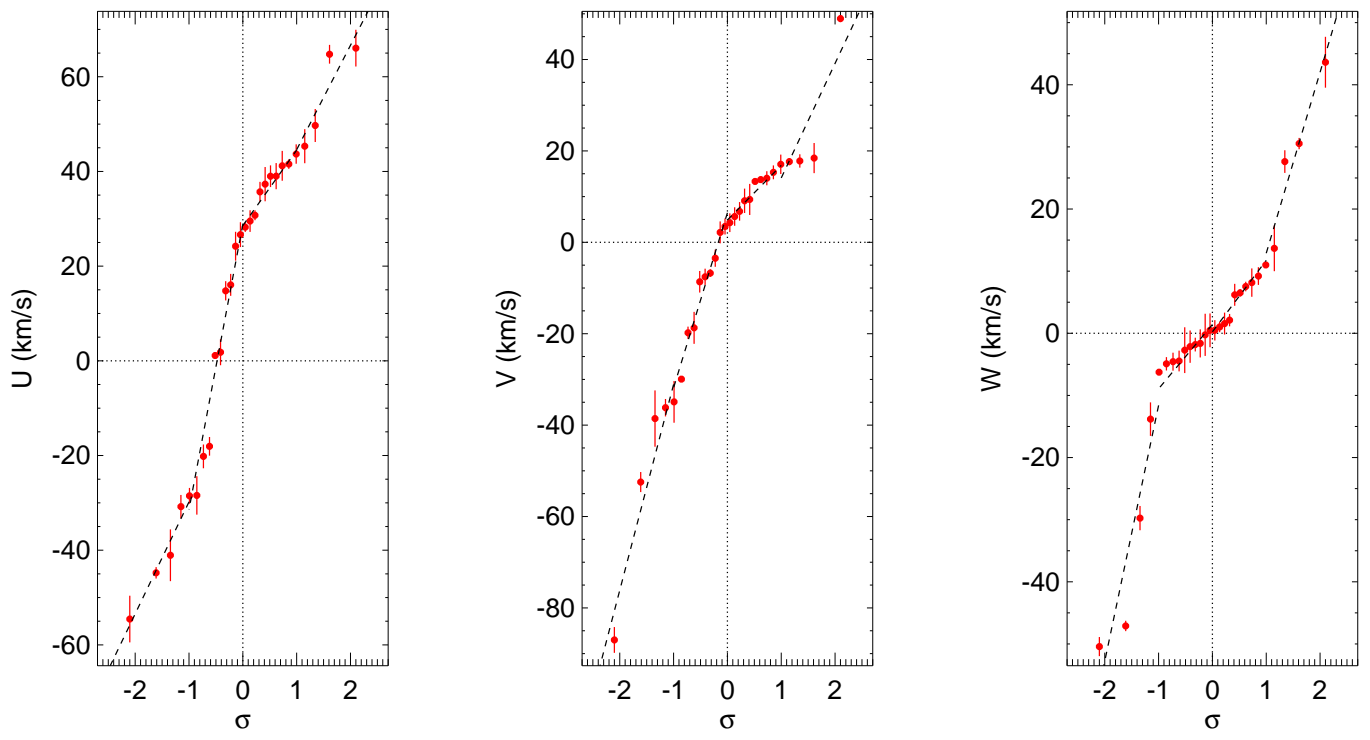

Fig. 13. - Same as Figure 11 for the L dwarfs in our sample. In this case, separate fits are made within the core population for $\sigma>0$ and $\sigma<0$, which have significantly different linear slopes. 

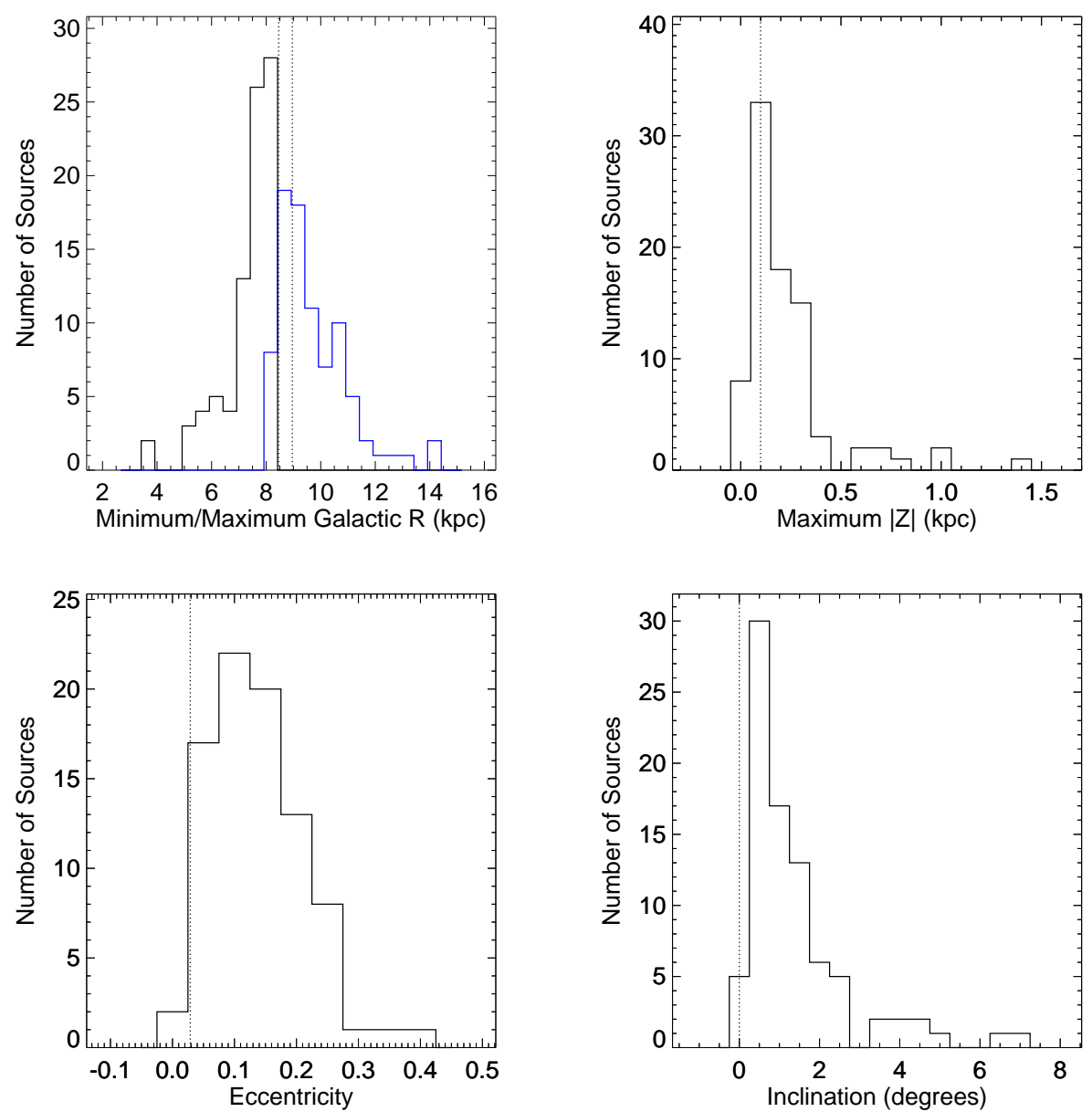

Fig. 14.- Distribution of Galactic orbital parameters for sources in our sample: (upper left) minimum (black) and maximum (blue) Galactic radius, (upper right) maximum absolute vertical displacement, (lower left) orbital eccentricity, and (lower right) orbital inclination. Solar values based on the same orbit calculations and assuming the current position $\left(R_{\odot}=8.43 \mathrm{kpc},\left|Z_{\odot}\right|=\right.$ $0.027 \mathrm{kpc}$ ) and motion of the Sun relative to the LSR are indicated by the dashed lines. 

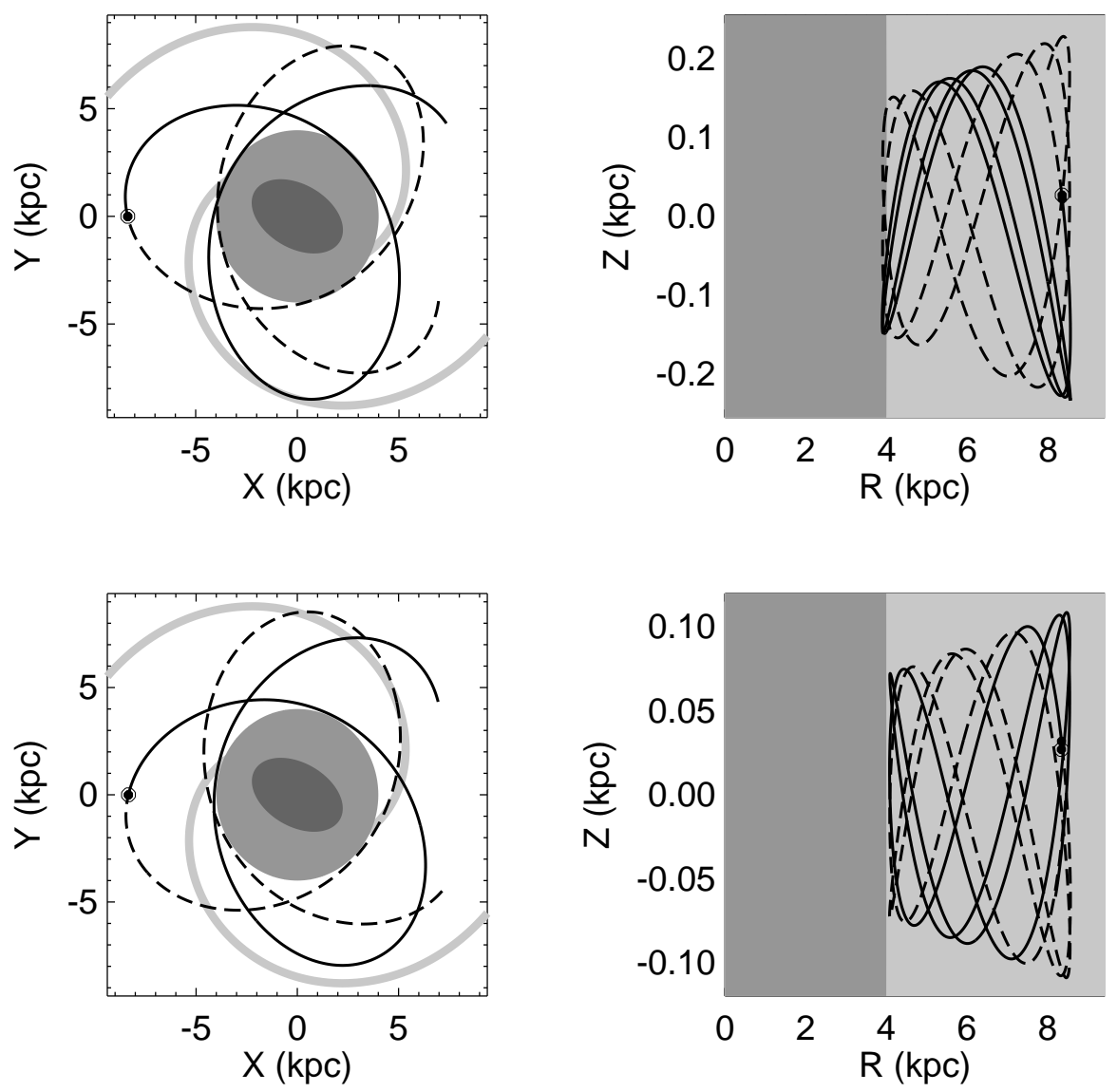

Fig. 15. - Galactic orbits of the thick disk M8.5 J0707-4900 (top) and the intermediate thin/thick disk L1 J0921-2104 (bottom). Panels show the orbit over the past (dashed line) and future (solid line) $250 \mathrm{Myr}$ about the current epoch (solid point, near the current position of the Sun), projected onto the Galactic plane (left) and in cylindrical coordinates (right). Representations of the Galactic bar (darkest gray), bulge (gray), and thin disk/major spiral arms (lightest gray) based on Benjamin (2008); Binney \& Tremaine (2008) and Wegg \& Gerhard (2013) are also shown. 

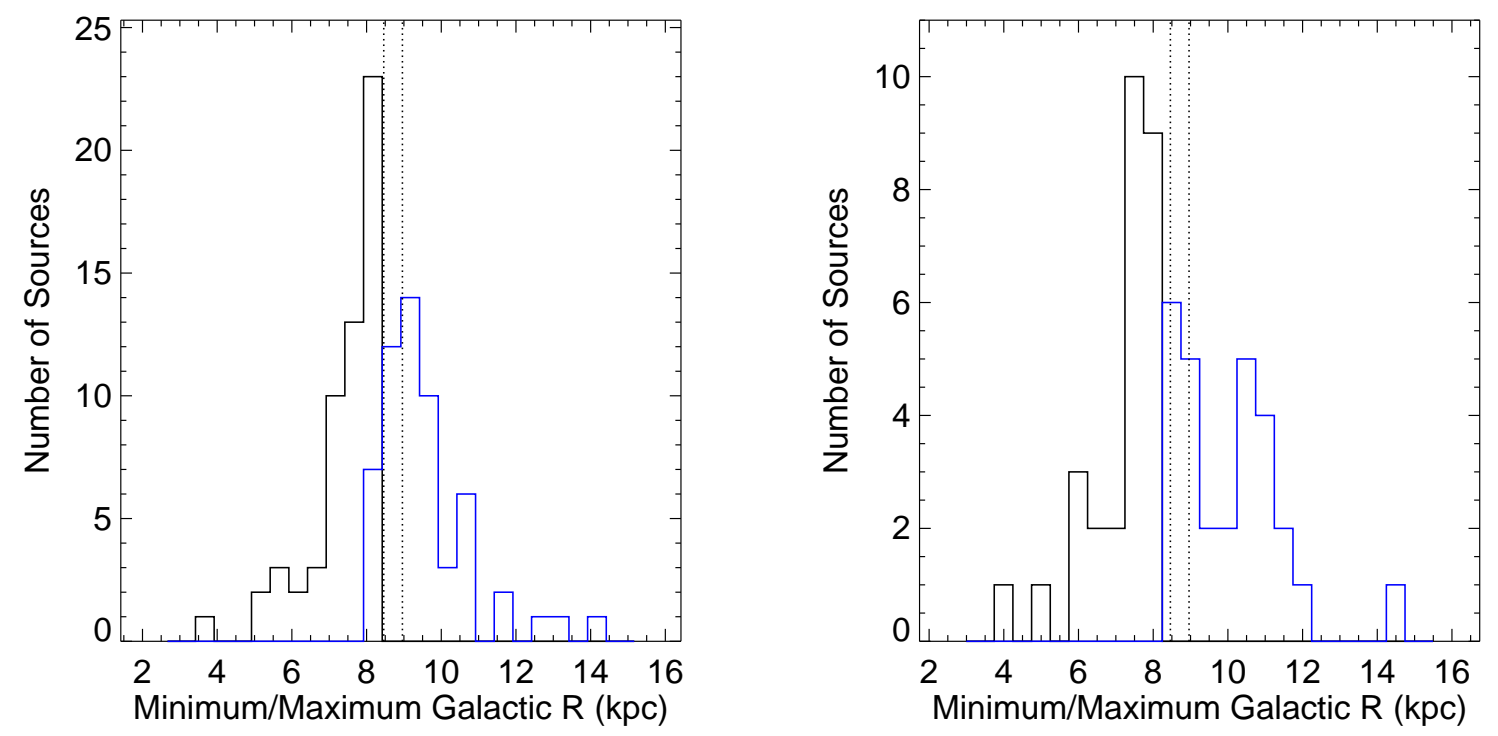

Fig. 16. - Comparison of $R_{\min }$ (black) and $R_{\max }$ (blue) distributions for the late-M dwarfs (left) and L dwarfs (right) in our sample. Solar values are indicated by vertical dashed lines. 

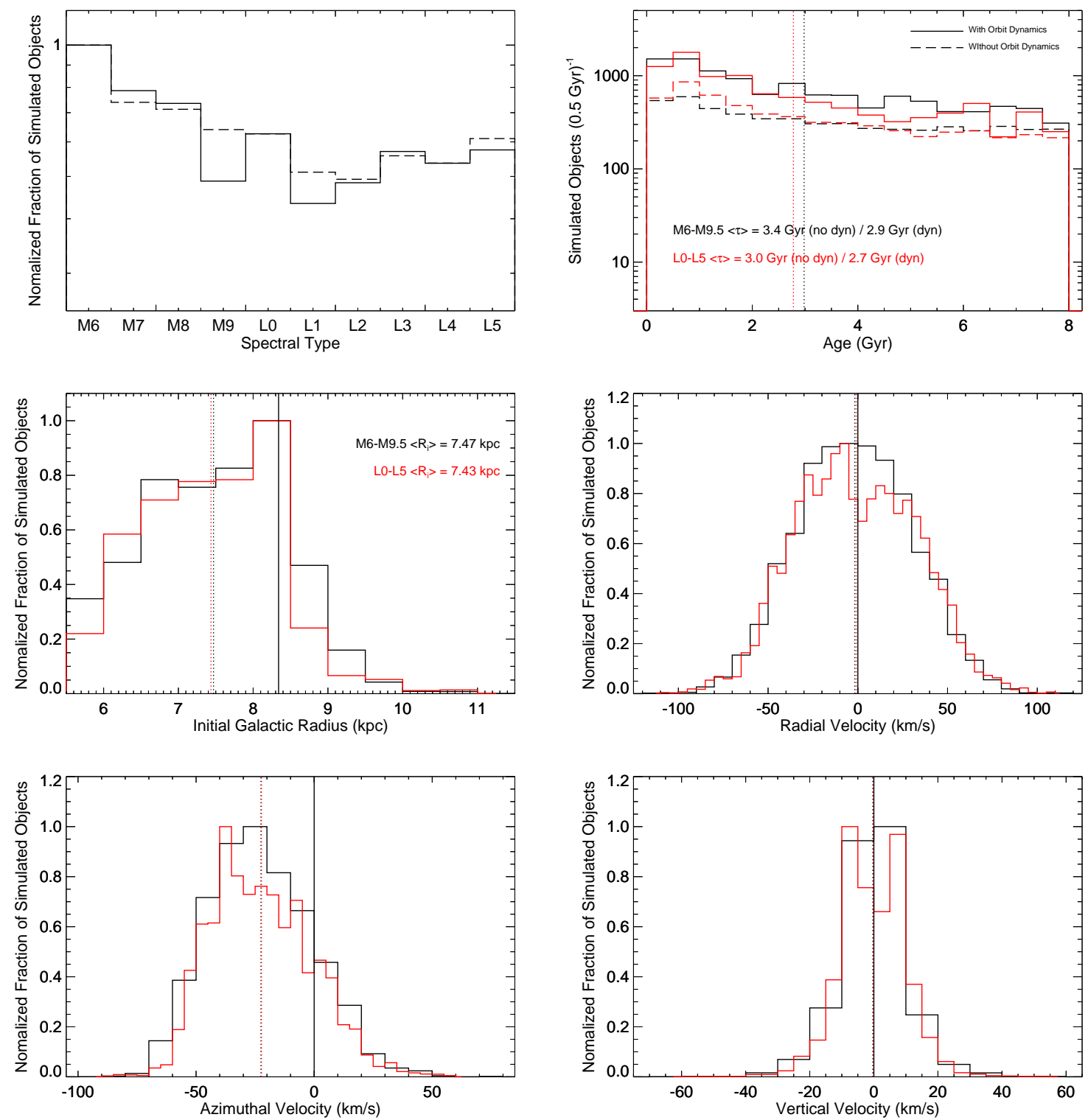

Fig. 17. - Summary of population synthesis, dynamical evolution and local selection simulation for $\beta=0.0$ and $\alpha=0.5$. Distributions for M6-M9.5 dwarfs are indicated in black, L0-L5 dwarfs in red. (Upper left): Spectral type distribution before (dashed) and after (solid) dynamical selection. (Upper right): Age distribution before (dashed) and after (solid) dynamical selection; vertical dotted lines indicate mean ages. (Middle left): Initial Galactic radii after dynamical selection. Dotted vertical lines indicate mean radii, solid vertical line indicates the Solar radius ( $8.43 \mathrm{kpc})$. (Middle right, lower left, lower right): Galactic azimuthal, radial and vertical velocities in the Local Standard of Rest after dynamical evolution and local selection. Vertical solid lines indicate zero mean velocity, vertical dotted lines indicate mean velocities of the spectral class subsamples. 

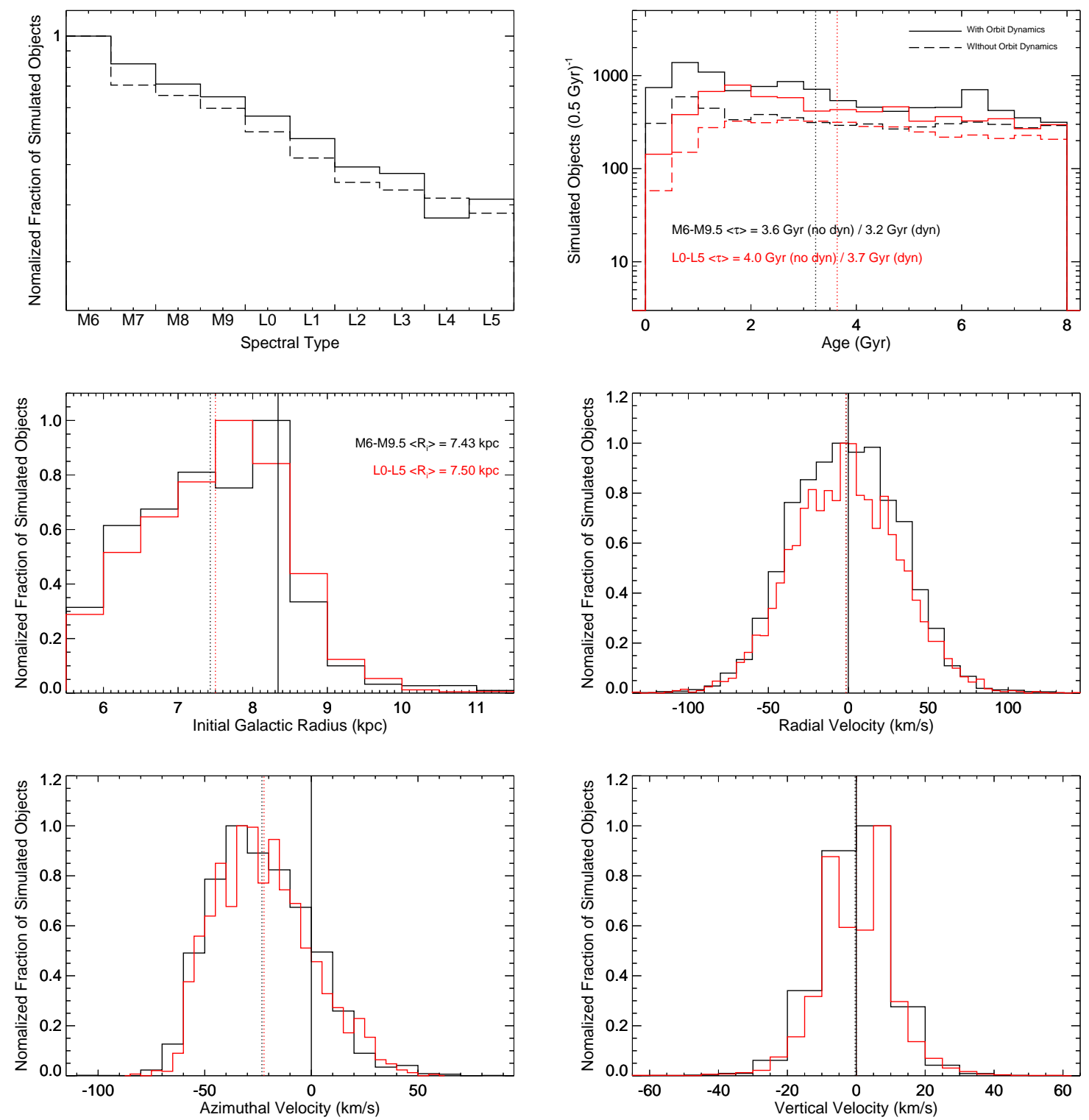

Fig. 18. - Same as Figure 17 but for $\beta=0.5$ for $\mathrm{M}<0.07 \mathrm{M}_{\odot}$ and $\beta=0.0$ for $\mathrm{M}>0.07 \mathrm{M}_{\odot}$. Note the loss of young L (brown) dwarfs in this sample, resulting in an older L dwarf population on average. 

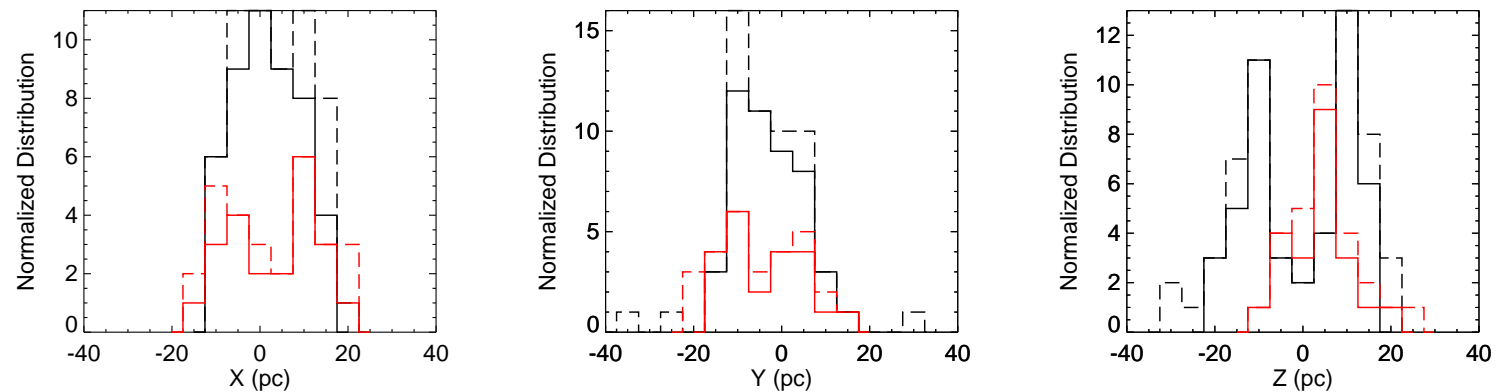

Fig. 19.- Distribution of $\mathrm{M}$ dwarfs (black) and $\mathrm{L}$ dwarfs (red) in $X Y Z$ spatial coordinates. Dashed-line histograms show the distributions for all sources, solid-line histograms show the distributions for those sources within 20 pc of the Sun.
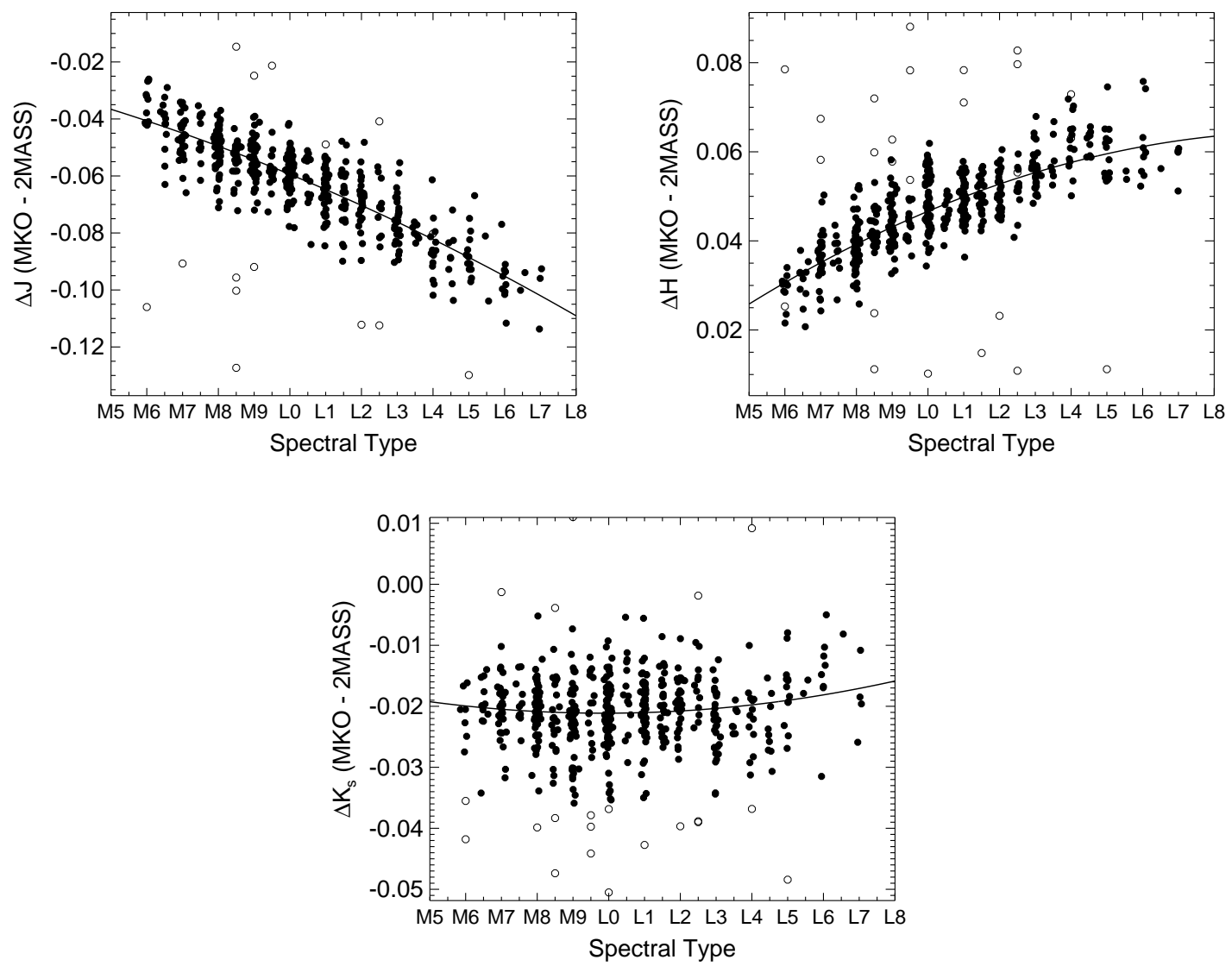

Fig. 20.- Magnitude differences between 2MASS and MKO $J H K_{s}$ filter systems for M6-L7 dwarfs, based on spectrophotometric measurements from 533 optically-classified, high S/N nearinfrared spectra in the SpeX Prism Library. Each point has a random offset in spectral type added to distinguish sources. Open circles are outliers rejected from the second-order polynomial fits. 
Table 1. Observational Sample

\begin{tabular}{|c|c|c|c|c|c|c|c|c|c|c|}
\hline $\begin{array}{l}\text { Source } \\
\text { Name }\end{array}$ & $\begin{array}{l}\text { Coordinates } \\
(\mathrm{J} 2000)\end{array}$ & $\begin{array}{l}\text { Optical } \\
\text { SpT }\end{array}$ & $\begin{array}{c}2 \mathrm{MASS} J \\
(\mathrm{mag})\end{array}$ & $\begin{array}{l}J-K_{s} \\
(\mathrm{mag})\end{array}$ & $\begin{array}{c}\mu_{\alpha} \\
\left(\operatorname{mas~}^{y^{-1}} \text { ) }\right.\end{array}$ & $\begin{array}{c}\mu_{\delta} \\
\left(\operatorname{mas~}^{-1} r^{-1}\right)\end{array}$ & $\begin{array}{r}d^{\mathrm{a}} \\
(\mathrm{pc})\end{array}$ & $\begin{array}{c}\text { Published } \\
R V \\
\left(\mathrm{~km} \mathrm{~s}^{-1}\right)\end{array}$ & Flag $^{\mathrm{b}}$ & References $^{c}$ \\
\hline J0004-2058 & 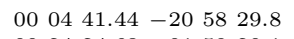 & M9 & $12.40 \pm 0.02$ & $1.01 \pm 0.03$ & $756 \pm 8$ & $95 \pm 10$ & $(15.5 \pm 1.0)$ & & & 28 \\
\hline J0024-0158 & 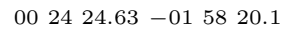 & M9.5 & $11.99 \pm 0.04$ & $1.45 \pm 0.04$ & $-80 \pm 4$ & $132 \pm 6$ & $11.6 \pm 0.5$ & $10 \pm 3$ & UR & $48 ; 51$ \\
\hline J0041-5621AB & 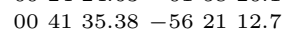 & M8 & $11.96 \pm 0.02$ & $1.10 \pm 0.03$ & $98 \pm 6$ & $-50 \pm 8$ & $(20.7 \pm 1.8)$ & $2.4 \pm 1$ & $\mathrm{Li}, \mathrm{VB}, \mathrm{THA}$ & $45 ; 55,51$ \\
\hline J0102-3737 & 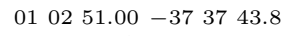 & M9 & $11.13 \pm 0.02$ & $1.06 \pm 0.03$ & $1456 \pm 3$ & $262 \pm 4$ & $12.2 \pm 0.4$ & & & 34 \\
\hline J0109-0343 & 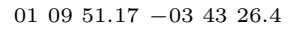 & M8 & $11.69 \pm 0.02$ & $1.27 \pm 0.03$ & $360 \pm 2$ & $18 \pm 2$ & $9.6 \pm 0.2$ & $-10.4 \pm 0.6$ & UR & $34 ; 55,51$ \\
\hline J0123-6921 & $\begin{array}{llllll}01 & 23 & 11.25 & -69 & 21 & 37.9\end{array}$ & M9 & $12.32 \pm 0.02$ & $1.00 \pm 0.04$ & $77 \pm 2$ & $-25 \pm 9$ & $42 \pm 5$ & $7.5 \pm 0.3$ & $\mathrm{Li}, \mathrm{THA}$ & $39 ; 55,51$ \\
\hline J0148-3024 & $014838.64-302439.6$ & M7.5 & $12.30 \pm 0.02$ & $1.08 \pm 0.03$ & $-86 \pm 8$ & $51 \pm 8$ & $(18.8 \pm 2.0)$ & $10 \pm 3$ & & $28 ; 51$ \\
\hline J0248-1651 & $0248 \quad 41.00-165121.6$ & M8 & $12.55 \pm 0.02$ & $1.13 \pm 0.03$ & $20 \pm 9$ & $-273 \pm 12$ & $16.1 \pm 1.3$ & $4 \pm 3$ & & $31 ; 51$ \\
\hline J0331-3042 & 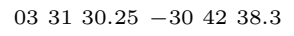 & M9 & $11.36 \pm 0.02$ & $1.10 \pm 0.03$ & $57 \pm 1$ & $-403 \pm 6$ & $(9.6 \pm 0.6)$ & $23 \pm 3$ & & $32 ; 51$ \\
\hline J0339-3525 & 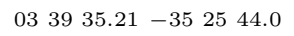 & M9 & $10.73 \pm 0.02$ & $1.18 \pm 0.03$ & $305.8 \pm 0.4$ & $270.5 \pm 0.4$ & $6.41 \pm 0.04$ & $6 \pm 3$ & $\mathrm{Li}, \mathrm{CAS}$ & $38 ; 56$ \\
\hline J0351-0052 & 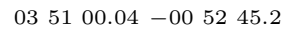 & M7.5 & $11.30 \pm 0.02$ & $1.07 \pm 0.03$ & $8.6 \pm 1.1$ & $-472.4 \pm 1$ & $14.7 \pm 0.4$ & $-15 \pm 3$ & & 42,$49 ; 51$ \\
\hline $\mathrm{J} 0500+0330$ & $050021.00+033050.1$ & $\mathrm{~L} 4$ & $13.67 \pm 0.02$ & $1.61 \pm 0.03$ & $13 \pm 3$ & $-349.9 \pm 1.8$ & $13.5 \pm 0.4$ & $15.94 \pm 0.16$ & UB & $1 ; 38 ; 52$ \\
\hline J0517-3349 & $\begin{array}{llllll}05 & 17 & 37.66 & -33 & 49 & 02.7\end{array}$ & M8 & $12.00 \pm 0.02$ & $1.17 \pm 0.03$ & $428 \pm 5$ & $-306 \pm 4$ & $(15.0 \pm 1.4)$ & $31 \pm 3$ & & $32 ; 51$ \\
\hline J0544-2433 & $\begin{array}{llllll}05 & 44 & 11.50 & -24 & 33 & 01.8\end{array}$ & M9 & $12.53 \pm 0.02$ & $1.07 \pm 0.03$ & $140 \pm 30$ & $-690 \pm 30$ & $(16.4 \pm 1.1)$ & $21 \pm 3$ & & $22 ; 51$ \\
\hline J0615-0100 & 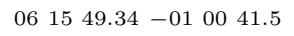 & L1 & $13.75 \pm 0.03$ & $1.21 \pm 0.04$ & $226 \pm 12$ & $-75 \pm 14$ & $(22.4 \pm 1.5)$ & $\ldots$ & & $2 ; 23$ \\
\hline J0641-4322 & 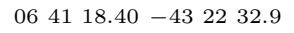 & L3 & $13.75 \pm 0.03$ & $1.30 \pm 0.04$ & $215 \pm 9$ & $611 \pm 9$ & $17.9 \pm 1.9$ & $\cdots$ & UB & $1 ; 24$ \\
\hline J0652-2534 & 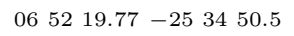 & M9 & $12.76 \pm 0.02$ & $1.24 \pm 0.03$ & $-235.6 \pm 0.8$ & $82.5 \pm 1.3$ & $15.7 \pm 0.2$ & & & $2 ; 38$ \\
\hline J0707-4900 & 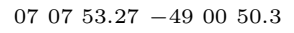 & M8.5 & $13.23 \pm 0.03$ & $1.12 \pm 0.04$ & $-10 \pm 5$ & $391 \pm 7$ & $16.4 \pm 0.8$ & $116.4 \pm 1.5$ & & 21,$49 ; 59$ \\
\hline J0751-2530 & $0751 \quad 16.45-253043.2$ & L1 & $13.16 \pm 0.02$ & $1.17 \pm 0.03$ & $-877.7 \pm 0.9$ & $142.2 \pm 1.4$ & $16.9 \pm 0.2$ & $\ldots$ & UB & 38 \\
\hline J0812-2444 & 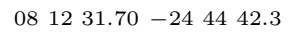 & $\mathrm{L} 2.5$ & $13.82 \pm 0.03$ & $1.43 \pm 0.04$ & $137.6 \pm 1.1$ & $-140.1 \pm 1.1$ & $22 \pm 0.5$ & & & $2 ; 38$ \\
\hline J0823-4912AB & 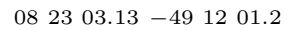 & L3 & $13.55 \pm 0.03$ & $1.49 \pm 0.04$ & $-154.30 \pm 0.12$ & $7.46 \pm 0.09$ & $20.76 \pm 0.08$ & $\ldots$ & $\mathrm{AB}$ & $2 ; 46$ \\
\hline J0830+0947 & $083032.56+094715.3$ & M7.5 & $11.89 \pm 0.02$ & $1.13 \pm 0.03$ & $-500 \pm 3$ & $-449 \pm 4$ & $16.9 \pm 1.3$ & $\ldots$ & & 35 \\
\hline J0835-0819 & 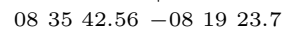 & L6 6 & $13.17 \pm 0.02$ & $2.03 \pm 0.03$ & $-520 \pm 9$ & $285 \pm 10$ & $8.5 \pm 0.8$ & $29.89 \pm 0.06$ & UR,HYA & $3 ; 24 ; 52,53$ \\
\hline J0847-1532 & 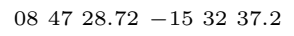 & $\mathrm{L} 2$ & $13.51 \pm 0.03$ & $1.45 \pm 0.04$ & $133.6 \pm 1.2$ & $-198.8 \pm 1$ & $17 \pm 0.3$ & $2.02 \pm 0.1$ & & $3 ; 38 ; 52$ \\
\hline J0853-0329 & $\begin{array}{llll}08 & 53 & 36.19 & -03 \\
29 & 32.1\end{array}$ & M9 & $11.21 \pm 0.03$ & $1.27 \pm 0.04$ & $-515.1 \pm 0.2$ & $-193.6 \pm 0.4$ & $8.48 \pm 0.05$ & $6 \pm 3$ & UR & $38 ; 51,56$ \\
\hline $\mathrm{J} 0902+0033$ & $0902 \quad 06.90+003319.5$ & M7 & $12.11 \pm 0.02$ & $0.95 \pm 0.03$ & $-466 \pm 3$ & $-97 \pm 3$ & $(19 \pm 2)$ & $48 \pm 5$ & & $26 ; 60$ \\
\hline J0909-0658 & $\begin{array}{llllll}09 & 09 & 57.49 & -06 & 58 & 18.6\end{array}$ & L1 & $13.89 \pm 0.02$ & $1.35 \pm 0.04$ & $-184 \pm 3$ & $21 \pm 3$ & $24 \pm 2$ & $27 \pm 3$ & & $4 ; 24 ; 56$ \\
\hline J0921-2104 & 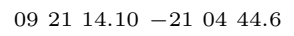 & L1 & $12.78 \pm 0.02$ & $1.09 \pm 0.03$ & $244 \pm 16$ & $-908 \pm 17$ & $(14.3 \pm 1.0)$ & $80.54 \pm 0.11$ & UB & $1 ; 21 ; 52,53$ \\
\hline $\mathrm{J} 0923+2340$ & $092308.61+234015.2$ & L1 & $13.85 \pm 0.03$ & $1.04 \pm 0.04$ & $294 \pm 36$ & $-396 \pm 20$ & $(23.4 \pm 1.6)$ & $-30 \pm 4$ & UB & $27 ; 57$ \\
\hline J0949+0806 & $094922.23+080645.0$ & M8.5 & $12.31 \pm 0.02$ & $1.10 \pm 0.04$ & $40.2 \pm 1.9$ & $-885.8 \pm 1.2$ & $16.6 \pm 0.5$ & & & 38 \\
\hline $\mathrm{J} 1003-0105$ & $\begin{array}{llllll}10 & 03 & 19.18 & -01 & 05 & 07.9\end{array}$ & M8 & $12.33 \pm 0.02$ & $1.09 \pm 0.03$ & $-490 \pm 19$ & $22 \pm 19$ & $(17.3 \pm 1.5)$ & $35 \pm 5$ & & $41 ; 60$ \\
\hline $\mathrm{J} 1004-3335$ & 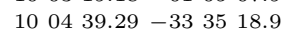 & L4 & $14.48 \pm 0.04$ & $1.56 \pm 0.04$ & $243 \pm 4$ & $-253 \pm 4$ & $18.2 \pm 2$ & & UB & $5 ; 24$ \\
\hline $\mathrm{J} 1045-0149$ & $104524.00-014957.6$ & $\mathrm{~L} 2$ & $13.16 \pm 0.02$ & $1.38 \pm 0.03$ & $-492 \pm 9$ & $-5 \pm 9$ & $(14.9 \pm 1.2)$ & $6.31 \pm 0.1$ & HYA & $5 ; 28 ; 52,53$ \\
\hline $\mathrm{J} 1048-3956$ & 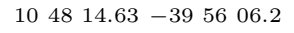 & M8 & $9.54 \pm 0.02$ & $1.09 \pm 0.03$ & $-1176 \pm 5$ & $-993 \pm 5$ & $4 \pm 0.03$ & $-13 \pm 3$ & & $34 ; 51$ \\
\hline $\mathrm{J} 1054+1214$ & $105441.68+121408.4$ & M7.5 & $12.46 \pm 0.02$ & $1.01 \pm 0.03$ & $103 \pm 18$ & $-80 \pm 19$ & $(20 \pm 2)$ & & & 21 \\
\hline $\mathrm{J} 1055+0808$ & $105547.33+080842.7$ & M9 & $12.55 \pm 0.03$ & $1.18 \pm 0.04$ & $-350 \pm 20$ & $-100 \pm 30$ & $(16.6 \pm 1.1)$ & $19 \pm 5$ & & $22 ; 60$ \\
\hline $\mathrm{J} 1119+0828$ & $111946.47+082035.6$ & M7 & $12.77 \pm 0.02$ & $0.87 \pm 0.04$ & $368 \pm 11$ & $-336 \pm 10$ & $(26 \pm 3)$ & $-39 \pm 5$ & & $28 ; 60$ \\
\hline J1121-1313AB & $\begin{array}{llllll}11 & 21 & 49.24 & -13 & 13 & 08.4\end{array}$ & M8.5 & $11.93 \pm 0.02$ & $1.19 \pm 0.03$ & $-464.2 \pm 0.7$ & $-57.0 \pm 0.7$ & $15.2 \pm 0.5$ & $32 \pm 3$ & $\mathrm{VB}, \mathrm{RV}$ & $42 ; 51$ \\
\hline $\mathrm{J} 1134+0022$ & $113454.93+002254.1$ & M9 & $12.85 \pm 0.02$ & $1.18 \pm 0.03$ & $401 \pm 21$ & $-343 \pm 21$ & $(19.0 \pm 1.3)$ & & & 21 \\
\hline J1139-3159 & 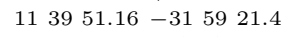 & M9 & $12.69 \pm 0.03$ & $1.18 \pm 0.03$ & $-81 \pm 4$ & $-28 \pm 2$ & $42 \pm 5$ & $12 \pm 2$ & Li, TWA & $5 ; 50 ; 56$ \\
\hline J1155-3727 & $\begin{array}{llll}11 & 55 & 39.52 & -37 \\
27 & 35.0\end{array}$ & L3 & $12.81 \pm 0.02$ & $1.35 \pm 0.03$ & $67 \pm 5$ & $-778 \pm 5$ & $9.6 \pm 0.4$ & $45.47 \pm 0.11$ & UB & $1 ; 25 ; 52,53$ \\
\hline $\mathrm{J} 1221+0257$ & $122127.70+025719.8$ & Lo & $13.17 \pm 0.02$ & $1.22 \pm 0.04$ & $-115 \pm 30$ & $-18 \pm 27$ & $(19.5 \pm 1.2)$ & $-8.79 \pm 0.14$ & & $27 ; 52,53$ \\
\hline $\mathrm{J} 1224-1238$ & $12 \quad 2452.22-12 \quad 38 \quad 35.2$ & M9 & $12.57 \pm 0.02$ & $1.22 \pm 0.04$ & $-263 \pm 11$ & $-187 \pm 11$ & $17 \pm 1.2$ & $-6 \pm 3$ & & $31 ; 51$ \\
\hline $\mathrm{J} 1252+0252$ & $125222.64+025205.8$ & M7 & $12.50 \pm 0.02$ & $0.99 \pm 0.04$ & $36 \pm 4$ & $-278 \pm 4$ & $(23 \pm 3)$ & $30 \pm 5$ & & $26 ; 60$ \\
\hline $\mathrm{J} 1309-2330$ & 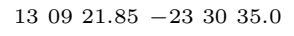 & M8 & $11.79 \pm 0.02$ & $1.12 \pm 0.03$ & $26.5 \pm 1.7$ & $-379.5 \pm 1.1$ & $14.4 \pm 0.3$ & $19 \pm 3$ & & $6 ; 38 ; 51$ \\
\hline $\mathrm{J} 1315-2649 \mathrm{AB}$ & $131530.94-26495.13$ & L5 & $15.20 \pm 0.05$ & $1.73 \pm 0.07$ & $-682 \pm 13$ & $-282 \pm 14$ & $(22 \pm 2)$ & $\ldots$ & VB & 21 \\
\hline $\mathrm{J} 1332-0441$ & $\begin{array}{llll}13 & 32 & 24.42 & -04 \\
41 & 12.6\end{array}$ & M7.5 & $12.37 \pm 0.03$ & $1.09 \pm 0.03$ & $59 \pm 9$ & $14 \pm 9$ & $(19 \pm 2)$ & $-17 \pm 3$ & & $28 ; 51$ \\
\hline $\mathrm{J} 1411-2119$ & 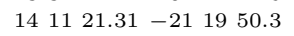 & M9 & $12.44 \pm 0.02$ & $1.11 \pm 0.03$ & $-73 \pm 8$ & $-70 \pm 8$ & $(15.8 \pm 1.1)$ & $-1 \pm 3$ & $\mathrm{Li}$ & $33 ; 51$ \\
\hline
\end{tabular}


Table 1 - Continued

\begin{tabular}{|c|c|c|c|c|c|c|c|c|c|c|}
\hline $\begin{array}{l}\text { Source } \\
\text { Name }\end{array}$ & $\begin{array}{l}\text { Coordinates } \\
(\mathrm{J} 2000)\end{array}$ & $\begin{array}{l}\text { Optical } \\
\text { SpT }\end{array}$ & $\underset{(\mathrm{mag})}{2 \mathrm{MASS}} J$ & $\begin{array}{l}J-K_{s} \\
(\mathrm{mag})\end{array}$ & $\begin{array}{c}\mu_{\alpha} \\
\left(\operatorname{mas~yr}^{-1} \text { ) }\right.\end{array}$ & $\begin{array}{c}\mu_{\delta} \\
\left(\operatorname{mas~yr}^{-1}\right)\end{array}$ & $\begin{array}{r}d^{\mathrm{a}} \\
(\mathrm{pc})\end{array}$ & $\begin{array}{c}\text { Published } \\
R V \\
\left(\mathrm{~km} \mathrm{~s}^{-1}\right)\end{array}$ & Flag $^{\mathrm{b}}$ & References $^{c}$ \\
\hline $\mathrm{J} 1422+2116$ & $1422 \quad 24.24+21 \quad 1607.6$ & M7 & $12.44 \pm 0.03$ & $0.99 \pm 0.03$ & $93 \pm 4$ & $-3 \pm 4$ & $(22 \pm 3)$ & 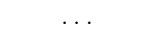 & & 26 \\
\hline $\mathrm{J} 1440+1339$ & $144022.93+133923.0$ & M7 & $12.40 \pm 0.02$ & $1.06 \pm 0.03$ & $-138.4 \pm 1.5$ & $-300.8 \pm 1.1$ & $22.2 \pm 0.6$ & $-5 \pm 3$ & & $38 ; 51$ \\
\hline J1456-2809 & 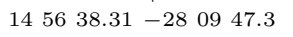 & M7 & $9.97 \pm 0.03$ & $1.04 \pm 0.04$ & $-470 \pm 10$ & $-844 \pm 12$ & $6.56 \pm 0.09$ & $1 \pm 3$ & & 48,$49 ; 51$ \\
\hline $\mathrm{J} 1500-0039$ & $150026.35-003928.1$ & M6 & $11.59 \pm 0.03$ & $0.95 \pm 0.04$ & $-186 \pm 3$ & $61 \pm 3$ & $(20 \pm 4)$ & $-17 \pm 5$ & & $26 ; 54$ \\
\hline $\mathrm{J} 1501+2250$ & $150108.18+225002.0$ & M9 & $11.87 \pm 0.02$ & $1.16 \pm 0.03$ & $-24.6 \pm 0.3$ & $-57.9 \pm 0.4$ & $10.59 \pm 0.07$ & $\ldots$ & & 36 \\
\hline J1504-2355 & $\begin{array}{llllll}15 & 04 & 16.21 & -23 & 55 & 56.4\end{array}$ & M7.5 & $12.01 \pm 0.03$ & $0.98 \pm 0.04$ & $-339 \pm 14$ & $-85 \pm 3$ & $(16.3 \pm 1.6)$ & & & $7 ; 29$ \\
\hline $\mathrm{J} 1506+1321$ & $150654.41+132106.0$ & L3 & $13.37 \pm 0.02$ & $1.62 \pm 0.03$ & $-1088 \pm 12$ & $4 \pm 10$ & $(13.8 \pm 1.3)$ & $-0.68 \pm 0.11$ & & $47 ; 52,53$ \\
\hline $\mathrm{J} 1510-0241$ & 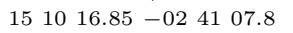 & M9 & $12.61 \pm 0.02$ & $1.27 \pm 0.03$ & $-404 \pm 12$ & $24 \pm 6$ & $16.4 \pm 1.3$ & $\ldots$ & UR & 48 \\
\hline $\mathrm{J} 1510-2818$ & $\begin{array}{llllll}15 & 10 & 47.86 & -28 & 18 & 17.4\end{array}$ & M9 & $12.84 \pm 0.03$ & $1.15 \pm 0.04$ & $-124 \pm 5$ & $-46 \pm 5$ & $(19.0 \pm 1.3)$ & $\ldots$ & & 43 \\
\hline J1534-1418 & $153457.04-14 \quad 1848.6$ & M8 & $11.38 \pm 0.02$ & $1.08 \pm 0.03$ & $-915 \pm 3$ & $-311.0 \pm 1$ & $(11.2 \pm 1.0)$ & $-76 \pm 3$ & & $32 ; 51$ \\
\hline $\mathrm{J} 1539-0520$ & $153941.89-052042.8$ & $\mathrm{~L} 4$ & $13.92 \pm 0.03$ & $1.35 \pm 0.04$ & $592.9 \pm 1.1$ & $105.6 \pm 1.8$ & $16.3 \pm 0.4$ & $27.3 \pm 0.2$ & UB & $8 ; 38 ; 52$ \\
\hline $\mathrm{J} 1550+1455 \mathrm{AB}$ & $155008.40+145517.1$ & L3.5 & $14.78 \pm 0.04$ & $1.52 \pm 0.06$ & $116 \pm 26$ & $-169 \pm 14$ & $(34 \pm 3)$ & $\ldots$ & VB & 27 \\
\hline $\mathrm{J} 1553+1400$ & $1553 \quad 19.93+140033.7$ & M9 & $13.05 \pm 0.02$ & $1.23 \pm 0.03$ & $-643 \pm 8$ & $66 \pm 9$ & $(20.9 \pm 1.5)$ & $\ldots$ & & $5 ; 28$ \\
\hline J1555-0956 & $1555 \quad 15.73-095605.5$ & L1 1 & $12.56 \pm 0.02$ & $1.11 \pm 0.03$ & $933.5 \pm 1.9$ & $-781 \pm 2$ & $13.4 \pm 0.2$ & $14.84 \pm 0.1$ & & $38 ; 52,53$ \\
\hline $\mathrm{J} 1607-0442$ & $160731.23-044209.1$ & M9 & $11.90 \pm 0.02$ & $1.18 \pm 0.03$ & $-1.4 \pm 1.9$ & $-414.6 \pm 1.2$ & $15.7 \pm 0.4$ & & & 38 \\
\hline $\mathrm{J} 1615+0546$ & $161542.45+054640.0$ & M9 & $12.88 \pm 0.02$ & $1.14 \pm 0.03$ & $136 \pm 5$ & $-81 \pm 5$ & $(19.2 \pm 1.3)$ & $\ldots$ & & $5 ; 44$ \\
\hline $\mathrm{J} 1645-1319$ & $\begin{array}{llllll}16 & 45 & 22.11 & -13 & 19 & 51.6\end{array}$ & L1.5 & $12.45 \pm 0.03$ & $1.31 \pm 0.04$ & $-352.6 \pm 1.1$ & $-799.5 \pm 0.7$ & $11.1 \pm 0.1$ & $26.58 \pm 0.06$ & & $9 ; 38 ; 52,53$ \\
\hline $\mathrm{J} 1705-0516$ & $170548.34-051646.2$ & L0.5 & $13.31 \pm 0.03$ & $1.28 \pm 0.04$ & $121.5 \pm 1.7$ & $-111.3 \pm 1.8$ & $18.2 \pm 0.6$ & $12.19 \pm 0.11$ & & $10 ; 38 ; 52,53$ \\
\hline $\mathrm{J} 1707-0558 \mathrm{AB}$ & $1707 \quad 23.43-05 \quad 58 \quad 24.9$ & LO & $12.05 \pm 0.02$ & $1.34 \pm 0.03$ & $100 \pm 8$ & $3 \pm 5$ & $(16.5 \pm 1.0)$ & $\ldots$ & $\mathrm{VB}, \mathrm{UMA}$ & $2 ; 40$ \\
\hline J1733-1654 & $173342.27-165450.0$ & L0.5 & $13.53 \pm 0.05$ & $1.18 \pm 0.06$ & $81 \pm 15$ & $-48 \pm 15$ & $(21.6 \pm 1.4)$ & $\ldots$ & & $2 ; 23$ \\
\hline $\mathrm{J} 1745-1640$ & $174534.66-16 \quad 4053.8$ & L1.5 & $13.65 \pm 0.03$ & $1.24 \pm 0.04$ & $116 \pm 5$ & $-111 \pm 19$ & $(20.0 \pm 1.4)$ & $\ldots$ & UB & $11 ; 23$ \\
\hline $\mathrm{J} 1750-0016$ & $175024.84-001615.1$ & L5.5 & $13.29 \pm 0.02$ & $1.45 \pm 0.03$ & $-398 \pm 3$ & $195 \pm 3$ & $9.2 \pm 0.2$ & $\ldots$ & UB & $12 ; 24$ \\
\hline J1845-6357 & 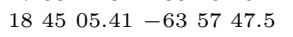 & M8.5 & $9.54 \pm 0.02$ & $1.04 \pm 0.03$ & $2591.9 \pm 1.8$ & $617 \pm 3$ & $3.85 \pm 0.02$ & $\ldots$ & & 30 \\
\hline $\mathrm{J} 2000-7523$ & 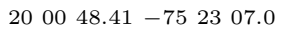 & M9 & $12.73 \pm 0.03$ & $1.22 \pm 0.04$ & $60.3 \pm 1.9$ & $-106 \pm 10$ & $(18.0 \pm 1.2)$ & $11.8 \pm 1$ & BPIC & $1 ; 28 ; 55$ \\
\hline $\mathrm{J} 2036+1051$ & $203603.16+105129.5$ & L3 & $13.95 \pm 0.03$ & $1.50 \pm 0.04$ & $-132 \pm 17$ & $-184 \pm 18$ & $(18.1 \pm 1.6)$ & $19.7 \pm 0.5$ & & $21 ; 52$ \\
\hline $\mathrm{J} 2037-1137$ & $203707.15-113756.9$ & M8 & $12.27 \pm 0.03$ & $1.02 \pm 0.03$ & $2 \pm 4$ & $-374 \pm 8$ & $(16.9 \pm 1.5)$ & $-39 \pm 3$ & & $43 ; 51$ \\
\hline $\mathrm{J} 2045-6332$ & $204502.38-63 \quad 3206.6$ & M9 & $12.62 \pm 0.03$ & $1.41 \pm 0.04$ & $82 \pm 3$ & $-204.3 \pm 1.7$ & $24 \pm 0.9$ & $0.5 \pm 0.5$ & $\mathrm{Li}, \mathrm{UR}, \mathrm{CAS}$ & $3 ; 38 ; 55$ \\
\hline J2104-1037 & 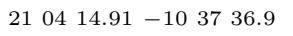 & L3 & $13.84 \pm 0.03$ & $1.47 \pm 0.04$ & $594.8 \pm 1.6$ & $-290 \pm 2$ & $18.9 \pm 0.6$ & $-21.09 \pm 0.12$ & & $38 ; 52,53$ \\
\hline $\mathrm{J} 2120+1021$ & $212033.87+102159.1$ & M8 & $13.54 \pm 0.03$ & $1.12 \pm 0.04$ & $121 \pm 10$ & $-30 \pm 11$ & $(30 \pm 3)$ & $-31 \pm 5$ & & $9 ; 28 ; 60$ \\
\hline $\mathrm{J} 2200-3038 \mathrm{AB}$ & $2200 \quad 02.01-3038 \quad 32.7$ & M8 & $13.44 \pm 0.03$ & $1.24 \pm 0.04$ & $210 \pm 48$ & $-64 \pm 21$ & $(41 \pm 4)$ & $-25 \pm 0.14$ & VB, UR, HYA & $40 ; 55,53$ \\
\hline $\mathrm{J} 2226-7503$ & $222644.40-750342.5$ & M8 & $12.35 \pm 0.02$ & $1.11 \pm 0.03$ & $48 \pm 19$ & $14 \pm 19$ & $(17.4 \pm 1.5)$ & $15 \pm 3$ & & $41 ; 51$ \\
\hline $\mathrm{J} 2306-0502$ & 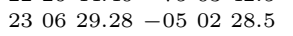 & M8 & $11.35 \pm 0.02$ & $1.06 \pm 0.03$ & $922 \pm 2$ & $-472 \pm 3$ & $12.1 \pm 0.4$ & $-52.8 \pm 0.16$ & & $35 ; 58,51$ \\
\hline $\mathrm{J} 2331-2749$ & $233121.74-274950.0$ & M7.5 & $11.65 \pm 0.02$ & $1.00 \pm 0.04$ & $77 \pm 2$ & $759.7 \pm 1.3$ & $14.5 \pm 0.4$ & $-4 \pm 3$ & & $35 ; 51$ \\
\hline $\mathrm{J} 2337-0838$ & $23 \quad 37 \quad 14.91-083808.4$ & M8 & $12.19 \pm 0.03$ & $1.00 \pm 0.04$ & $248 \pm 19$ & $17 \pm 19$ & $(16.3 \pm 1.4)$ & & & 41 \\
\hline $\mathrm{J} 2346+1129$ & $234645.99+112909.4$ & M8 & $12.80 \pm 0.02$ & $1.19 \pm 0.03$ & $-392 \pm 8$ & $-82 \pm 4$ & $(21.5 \pm 1.9)$ & $\ldots$ & UR & 42 \\
\hline $\mathrm{J} 2349+1224$ & $234948.99+122438.6$ & M8 & $12.60 \pm 0.02$ & $1.04 \pm 0.03$ & $18 \pm 19$ & $-209 \pm 19$ & $(19.6 \pm 1.6)$ & $-2 \pm 0.1$ & & $41 ; 58,51$ \\
\hline $\mathrm{J} 2351-2537$ & 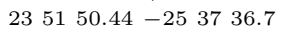 & M8 & $12.47 \pm 0.03$ & $1.20 \pm 0.04$ & $387 \pm 21$ & $163 \pm 9$ & $(18.5 \pm 1.7)$ & $-3 \pm 1.1$ & UR & $37 ; 53,51$ \\
\hline
\end{tabular}

${ }^{\mathrm{a}}$ Distances in parentheses are spectrophotometric estimates based on the $M_{J} /$ spectral type relation of Cruz et al. (2003).

bSource flags: $(\mathrm{VB})=$ visual binary, $(\mathrm{RV})=$ radial velocity variable, $(\mathrm{AB})=$ astrometric binary, $(\mathrm{Li})=$ previous reported Li I absorption, $(\mathrm{UB})=$ unusually blue, $(\mathrm{UR})=$ unusually red, $(\beta \mathrm{PMG})=$ previously identified member of $\beta$ Pictoris Moving Group, $(\mathrm{CAS})=$ previously identified member of Caster Moving Group, (HYA) $=$ previously identified member of Hyades Moving Group, (THA) = previously identified member of Tucana Horologium Association, (TWA) = previously identified member of TW Hydrae Association, (UMA) =

${ }^{\mathrm{b}}$ References in order of source discovery, astrometry and previously published RV measurement.

References. - Source discovery: (1) Reid et al. 2008; (2) Phan-Bao et al. 2008; (3) Cruz et al. 2003; (4) Delfosse et al. 1999; (5) Gizis 2002; (6) Hall 2002b; (7) Gizis et al. 
2000; (8) Cruz et al. 2007; (9) Kendall et al.|2004; (10) McElwain \& Burgasser|2006; (11) Kendall et al. 2007; (12) Biller et al.|2006; Source astrometry: (21) Faherty et al. 2009; (22) chmidt et al. 2007; (23) Phan-Bao et al. 2008; (24) Andrei et al. 2011; (25) Faherty et al. (2012); (26) York et al. 2000; (27) Schmidt et al. 2010; (28) This paper; (29) Lodieu et al. 2005; (30) Henry et al.||2006; (31) Tinney 1996; (32) Bartlett (2007); (33) Caballero (2007); (34) Costa et al. (2005); (35) Costa et al. 2006; (36) Dahn et al. (2002); (37) Deacon \& Hambly

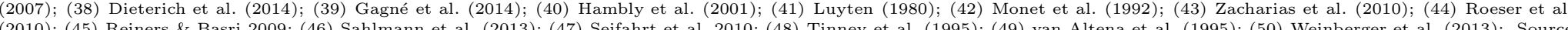
RV measurements: (51) Reiners \& Basri| 2009; (52) Blake et al. 2010; (53) Seifahrt et al. 2010; (54) Bochanski et al. (2005); (55) Gálvez-Ortiz et al. (2010); (56) Mohanty \& Basri (2003); (57) Schmidt et al. (2010); (58) Tanner et al. $(2012)$; (59) Tinney \& Reid (1998); (60) West et al. (2008). 
Table 2. Observations

\begin{tabular}{|c|c|c|c|c|c|}
\hline Source & $\begin{array}{l}\text { Observation } \\
\text { Date (UT) }\end{array}$ & $\begin{array}{c}\text { Integration } \\
\text { Time (s) }\end{array}$ & Airmass & $\begin{array}{c}\text { Flux } \\
\text { Calibrator }\end{array}$ & Conditions/Seeing \\
\hline J0004-2058 & 2009 Jan 11 & 800 & 1.54 & Hiltner 600 & seeing $\sim 00^{\prime \prime} 6$ \\
\hline J0024-0158 & 2009 Aug 27 & 450 & 1.179 & Feige 110 & dry, clear, windy, seeing $\sim 1$ \\
\hline J0041-5621AB & 2009 Aug 28 & 600 & 1.329 & Feige 110 & seeing $\sim 00^{\prime \prime} 7-0 .{ }^{\prime \prime} 8$ \\
\hline J0102-3737 & 2008 Nov 26 & 600 & 1.18 & HR 3454 & seeing $\sim 0 . \prime 8-0 . \prime 9$ \\
\hline J0109-0343 & 2009 Aug 27 & 450 & 1.12 & Feige 110 & dry, clear, windy, seeing $\sim 1$ \\
\hline J0123-6921 & 2009 Aug 28 & 600 & 1.427 & Feige 110 & seeing $\sim 00^{\prime \prime} 7$ \\
\hline J0148-3024 & 2009 Aug 29 & 600 & 1.005 & Feige 110 & clear; seeing $\quad 0^{\prime \prime} 5$ \\
\hline J0248-1651 & 2009 Aug 27 & 600 & 1.055 & Feige 110 & dry, clear, windy, seeing $\sim 0{ }^{\prime \prime} 7$ \\
\hline J0331-3042 & 2009 Aug 29 & 300 & 1.009 & Feige 110 & clear; seeing $\sim 00^{\prime \prime} 7$ \\
\hline J0339-3525 & 2009 Aug 29 & 300 & 1.015 & Feige 110 & clear; in twilight; seeing $\sim 00^{\prime \prime} 7$ \\
\hline J0351-0052 & 2009 Aug 28 & 300 & 1.138 & Feige 110 & in twilight, seeing $\sim 0^{\prime \prime} 7$ \\
\hline $\mathrm{J} 0500+0330$ & 2009 Jan 06 & 2400 & 1.195 & Hiltner 600 & clear; seeing $\sim 0{ }^{\prime \prime} 75$ \\
\hline J0517-3349 & 2009 Jan 06 & 600 & 1.12 & Hiltner 600 & clear; seeing $\sim 0^{\prime \prime} 75$ \\
\hline J0544-2433 & 2009 Jan 06 & 800 & 1.17 & Hiltner 600 & clear; seeing $\sim 0^{\prime \prime} 75$ \\
\hline J0615-0100 & 2009 Jan 06 & 2400 & 1.13 & Hiltner 600 & clear; seeing $\sim 0^{\prime \prime} 75$ \\
\hline J0641-4322 & 2011 Mar 19 & 900 & 1.03 & Hiltner 600 & seeing $\sim 00^{\prime \prime} 6$ \\
\hline J0652-2534 & 2009 Jan 07 & 2400 & 1.5 & Hiltner 600 & seeing $\sim 1$ \\
\hline J0707-4900 & 2009 Jan 08 & 1400 & 1.43 & Hiltner 600 & seeing $\sim 1$ \\
\hline J0751-2540 & 2009 Jan 07 & 1800 & 1.1 & Hiltner 600 & seeing $\sim 1$ \\
\hline J0812-2444 & 2009 Jan 07 & 2400 & 1.06 & Hiltner 600 & seeing $\sim 1$ \\
\hline J0823-4912AB & 2009 Jan 07 & 1800 & 1.08 & Hiltner 600 & seeing $\sim 1$ \\
\hline J0830+0947 & 2009 Jan 09 & 450 & 1.39 & Hiltner 600 & seeing $\sim 0{ }^{\prime \prime} 8$ \\
\hline J0835-0819 & 2009 Jan 08 & 2000 & 1.15 & Hiltner 600 & seeing $\sim 0 . \prime 5$ \\
\hline \multirow[t]{2}{*}{ J0847-1532 } & 2009 Jan 08 & 2000 & 1.07 & Hiltner 600 & seeing $\sim 0{ }^{\prime \prime} 5$ \\
\hline & 2009 Jan 11 & 2000 & 1.58 & Hiltner 600 & seeing $\sim 0^{\prime \prime} 5$ \\
\hline J0853-0329 & 2009 Jan 08 & 450 & 1.12 & Hiltner 600 & seeing $\sim 0 . .5$ \\
\hline J0902+0033 & 2011 Mar 19 & 600 & 1.15 & Hiltner 600 & seeing $\sim 0 . \prime 8-0 . .9$ \\
\hline J0909-0658 & 2011 Mar 19 & 1400 & 1.1 & Hiltner 600 & seeing $\sim 1.2$ \\
\hline J0921-2104 & 2009 Jan 09 & 1500 & 1.14 & Hiltner 600 & seeing $\sim 00^{\prime \prime} 8$ \\
\hline J0923+2340 & 2011 Mar 20 & 1500 & 1.69 & Hiltner 600/EG 274 & seeing $\sim 0 !^{\prime \prime} 8$ \\
\hline J0949+0806 & 2009 Mar 06 & 1100 & 1.37 & GD 108 & seeing $\sim 0 .{ }^{\prime \prime} 8-1$ \\
\hline J1003-0105 & 2011 Mar 19 & 600 & 1.17 & Hiltner 600 & seeing $\sim 1$ \\
\hline J1004-3335 & 2011 Mar 19 & 1600 & 1.02 & Hiltner 600 & seeing $\sim 00^{\prime \prime} 9$ \\
\hline J1045-0149 & 2009 Jan 09 & 1800 & 1.45 & Hiltner 600 & seeing variable $\sim 0 . \prime 75$ \\
\hline J1048-3956 & 2009 Jan 09 & 150 & 1.03 & Hiltner 600 & seeing variable $\sim 0 . \prime 75$ \\
\hline $\mathrm{J} 1054+1214$ & 2009 Jan 09 & 600 & 1.34 & Hiltner 600 & seeing variable $\sim 0^{\prime \prime} 75$ \\
\hline $\mathrm{J} 1055+0808$ & 2009 Mar 06 & 900 & 1.31 & GD 108 & seeing $\sim 1$ \\
\hline $\mathrm{J} 1119+0828$ & 2011 Mar 19 & 600 & 1.37 & Hiltner 600 & some moon glare; seeing $\sim 1$ \\
\hline \multirow[t]{2}{*}{ J1121-1313AB } & 2009 Jan 11 & 600 & 1.85 & Hiltner 600 & seeing $\sim 00^{\prime \prime} 5$ \\
\hline & 2009 Mar 06 & 600 & 1.08 & GD 108 & seeing $\sim 1$ \\
\hline $\mathrm{J} 1134+0022$ & 2009 Mar 06 & 1200 & 1.19 & GD 108 & seeing $\sim 1$ \\
\hline J1139-3159 & 2009 Mar 06 & 1050 & 1.014 & GD 108 & seeing $\sim 0^{\prime \prime} 7$ \\
\hline J1155-3727 & 2009 Mar 06 & 1500 & 1.02 & GD 108 & seeing $\sim 0 !^{\prime \prime} 8$ \\
\hline $\mathrm{J} 1221+0257$ & 2009 Mar 06 & 1500 & 1.178 & GD 108 & seeing $\sim 0 . ! 6$ \\
\hline J1224-1238 & 2009 Mar 06 & 1050 & 1.046 & GD 108 & seeing $\sim 00^{\prime \prime} 65$ \\
\hline
\end{tabular}


Table 2-Continued

\begin{tabular}{|c|c|c|c|c|c|}
\hline Source & $\begin{array}{c}\text { Observation } \\
\text { Date (UT) }\end{array}$ & $\begin{array}{l}\text { Integration } \\
\text { Time (s) }\end{array}$ & Airmass & $\begin{array}{c}\text { Flux } \\
\text { Calibrator }\end{array}$ & Conditions/Seeing \\
\hline $\mathrm{J} 1252+0252$ & 2011 Mar 19 & 600 & 1.45 & Hiltner 600 & seeing $\sim 1$ \\
\hline J1309-2330 & 2009 Mar 06 & 450 & 1.005 & GD 108 & seeing $\sim 0{ }^{\prime \prime} 6$ \\
\hline J1315-2649AB & 2011 Mar 26 & 3000 & 1.003 & EG 274 & clear; seeing $\sim 0^{\prime \prime} 6$ \\
\hline J1332-0441 & 2009 Mar 06 & 600 & 1.097 & GD 108 & seeing $\sim 0 .^{\prime \prime} 5$ \\
\hline J1411-2119 & 2009 Mar 06 & 900 & 1.018 & GD 108 & seeing $\sim 0^{\prime \prime} 5$ \\
\hline $\mathrm{J} 1422+2116$ & 2011 Mar 19 & 900 & 1.6 & Hiltner 600 & seeing $\sim 1.5-1.6$ \\
\hline $\mathrm{J} 1440+1339$ & 2011 Mar 19 & 900 & 1.5 & Hiltner 600 & seeing $\sim 00^{\prime \prime} 7$ \\
\hline J1456-2809 & 2009 Mar 06 & 150 & 1.002 & GD 108 & seeing $\sim 0^{\prime \prime} 6$ \\
\hline J1500-0039 & 2011 Mar 19 & 600 & 1.25 & Hiltner 600 & seeing $\sim 1.25$ \\
\hline $\mathrm{J} 1501+2250$ & 2011 Mar 19 & 900 & 1.71 & Hiltner 600 & seeing $\sim 2$ \\
\hline J1504-2355 & 2009 Mar 06 & 450 & 1.004 & GD 108 & seeing $\sim 00^{\prime \prime} 62$ \\
\hline $\mathrm{J} 1506+1321$ & 2009 Mar 06 & 1800 & 1.354 & GD 108 & seeing $\sim 0.0^{\prime \prime} 7$ \\
\hline J1510-0241 & 2009 Mar 07 & 1050 & 1.13 & GD 108 & seeing $\sim 0{ }^{\prime \prime} 6$ \\
\hline J1510-2818 & 2009 Mar 07 & 1200 & 1.003 & GD 108 & seeing $\sim 00^{\prime \prime} 6$ \\
\hline J1534-1418 & 2009 Mar 07 & 300 & 1.036 & GD 108 & seeing $\sim 0^{\prime \prime} 5-00^{\prime \prime} 6$ \\
\hline J1539-0520 & 2011 Mar 19 & 1500 & 1.16 & Hiltner 600 & seeing 1.5-1.6 \\
\hline \multirow[t]{2}{*}{$\mathrm{J} 1550+1455 \mathrm{AB}$} & 2009 Aug 28 & 3600 & 1.47 & Feige 110 & seeing $\sim 1.3$ \\
\hline & 2009 Aug 29 & 3600 & 1.471 & Feige 110 & clear; $0{ }^{\prime \prime} 6-0 .{ }^{\prime \prime} 9$ \\
\hline $\mathrm{J} 1553+1400$ & 2009 Mar 08 & 1500 & 1.76 & GD 108 & seeing $\sim 1$ \\
\hline J1555-0956 & 2009 Mar 08 & 1200 & 1.145 & GD 108 & seeing $\sim 0 !^{\prime \prime} 4$ \\
\hline J1607-0442 & 2009 Mar 08 & 600 & 1.213 & GD 108 & seeing $\sim 0^{\prime \prime} 6$ \\
\hline $\mathrm{J} 1615+0546$ & 2009 Mar 08 & 1200 & 1.347 & GD 108 & seeing $\sim 0 !{ }^{\prime} 7$ \\
\hline J1645-1319 & 2009 Aug 29 & 1200 & 1.079 & GD 108 & seeing $\sim 0 . \prime 7-0 .{ }^{\prime \prime} 8$ \\
\hline J1705-0516 & 2009 Mar 08 & 1200 & 1.143 & Hiltner $600 /$ EG 274 & seeing $\sim 0 . \prime 7$ \\
\hline J1707-0558AB & 2011 Mar 20 & 2400 & 1.238 & Feige 110 & clear; variable seeing $\sim 0^{\prime \prime} 7$ \\
\hline J1733-1654 & 2009 Aug 29 & 3000 & 1.034 & Feige 110 & seeing $\sim 1.1$ \\
\hline J1745-1640 & 2009 May 31 & 3000 & 1.024 & EG 274 & seeing $\sim 00^{\prime \prime} 9$ \\
\hline J1750-0016 & 2009 Aug 29 & 2700 & 1.334 & Feige 110 & clear; seeing $\sim 1.1$ \\
\hline J1845-6357 & 2009 May 31 & 200 & 1.256 & EG 274 & seeing $\sim 1$ \\
\hline J2000-7523 & 2009 May 31 & 2000 & 1.449 & EG 274 & seeing $\sim 1$ \\
\hline $\mathrm{J} 2036+1051$ & 2009 May 31 & 2950 & 1.351 & EG 274 & windy; seeing $\sim 1.2$ \\
\hline J2037-1137 & 2009 Aug 27 & 750 & 1.062 & Feige 110 & dry, clear and windy; seeing $\sim 0^{\prime \prime} 75$ \\
\hline J2045-6332 & 2009 Aug 27 & 2700 & 1.272 & Feige 110 & dry, clear and windy; seeing $\sim 0{ }^{\prime \prime} 7$ \\
\hline J2104-1037 & 2009 Aug 27 & 2700 & 1.074 & Feige 110 & dry, clear and windy; seeing $\sim 0^{\prime \prime} 9$ \\
\hline $\mathrm{J} 2120+1021$ & 2009 Aug 29 & 2400 & 1.31 & Feige 110 & clear; seeing $\sim 0 !{ }^{\prime \prime} 8$ \\
\hline J2200-3038AB & 2009 Aug 29 & 2400 & 1.002 & Feige 110 & clear; seeing $\sim 0^{\prime \prime} 6$ \\
\hline J2226-7503 & 2009 May 31 & 1500 & 1.47 & EG 274 & seeing $\sim 1$ \\
\hline J2306-0502 & 2009 Aug 27 & 300 & 1.095 & Feige 110 & dry, clear and windy; seeing $\sim 0{ }^{\prime \prime} 8$ \\
\hline J2331-2749 & 2009 Aug 28 & 450 & 1.03 & Feige 110 & seeing $\sim 0 !^{\prime} 9$ \\
\hline J2337-0838 & 2009 Aug 27 & 450 & 1.073 & Feige 110 & dry, clear and windy; seeing $\sim 0^{\prime \prime} 9$ \\
\hline $\mathrm{J} 2346+1129$ & 2009 Aug 28 & 1200 & 1.336 & Feige 110 & seeing $\sim 0{ }^{\prime \prime} 8$ \\
\hline $\mathrm{J} 2349+1224$ & 2009 Aug 28 & 900 & 1.336 & Feige 110 & seeing $\sim 0{ }^{\prime \prime} 9$ \\
\hline J2351-2537 & 2009 Aug 28 & 600 & 1.033 & Feige 110 & seeing $\sim 0{ }^{\prime \prime} 7$ \\
\hline
\end{tabular}


Table 3. Equivalent Widths and Line Strengths of Absorption and Emission Lines

\begin{tabular}{|c|c|c|c|c|c|c|c|c|c|c|c|}
\hline Source & $\mathrm{SpT}$ & $\begin{array}{c}\mathrm{Li} \mathrm{I} \\
6710 \AA\end{array}$ & $\begin{array}{c}\mathrm{Rb} I \\
7802 \AA\end{array}$ & $\begin{array}{c}\mathrm{Rb} \mathrm{I} \\
7950 \AA\end{array}$ & $\begin{array}{c}\mathrm{Na} \mathrm{I} \\
8186 \AA\end{array}$ & $\begin{array}{c}\mathrm{NaI} \\
8197 \AA\end{array}$ & $\begin{array}{c}\text { Cs I } \\
8523 \AA\end{array}$ & $\begin{array}{c}\text { Cs I } \\
8946 \AA\end{array}$ & $\begin{array}{c}\mathrm{H} \alpha \\
6583 \AA\end{array}$ & $\log _{10} L_{H \alpha} / L_{b o l}$ & $\zeta$ \\
\hline J0004-2058 & M9 & $<1.3$ & $1.54 \pm 0.17$ & $1.24 \pm 0.15$ & $1.7 \pm 0.2$ & $2.1 \pm 0.3$ & $0.8 \pm 0.2$ & $<0.7$ & $-4.2 \pm 0.4$ & $-5.04 \pm 0.06$ & $0.973 \pm 0.018$ \\
\hline J0024-0158 & M9.5 & $<0.4$ & $1.62 \pm 0.16$ & $1.39 \pm 0.18$ & $1.49 \pm 0.18$ & $1.59 \pm 0.18$ & $1.01 \pm 0.14$ & $0.8 \pm 0.2$ & $-0.13 \pm 0.12$ & $-6.57 \pm 0.13$ & $0.56 \pm 0.03$ \\
\hline J0041-5621AB & M8 & $0.7 \pm 0.2$ & $0.94 \pm 0.09$ & $0.70 \pm 0.18$ & $1.59 \pm 0.12$ & $1.8 \pm 0.2$ & $0.55 \pm 0.08$ & $<0.7$ & $-22 \pm 3$ & $-4.01 \pm 0.06$ & $1.208 \pm 0.016$ \\
\hline J0102-3737 & M9 & $<0.2$ & $1.33 \pm 0.11$ & $0.98 \pm 0.16$ & $1.83 \pm 0.14$ & $2.2 \pm 0.2$ & $0.58 \pm 0.13$ & $<1.1$ & $-7.24 \pm 0.16$ & $-4.76 \pm 0.06$ & $1.030 \pm 0.004$ \\
\hline J0109-0343 & M8 & $<0.4$ & $1.03 \pm 0.12$ & $0.59 \pm 0.16$ & $1.47 \pm 0.17$ & $1.7 \pm 0.2$ & $0.56 \pm 0.11$ & $<0.9$ & $-3.58 \pm 0.12$ & $-4.82 \pm 0.06$ & $1.20 \pm 0.04$ \\
\hline J0123-6921 & M9 & $0.56 \pm 0.17$ & $0.93 \pm 0.09$ & $0.62 \pm 0.13$ & $1.40 \pm 0.10$ & $1.66 \pm 0.18$ & $<0.5$ & $<0.8$ & $-11.9 \pm 1.4$ & $-4.51 \pm 0.06$ & $1.28 \pm 0.03$ \\
\hline J0148-3024 & M7.5 & $<0.4$ & $1.33 \pm 0.14$ & $1.09 \pm 0.10$ & $1.7 \pm 0.2$ & $2.1 \pm 0.2$ & $0.37 \pm 0.04$ & $<0.6$ & $-7.8 \pm 0.8$ & $-4.40 \pm 0.06$ & $1.052 \pm 0.008$ \\
\hline J0248-1651 & M8 & $<0.5$ & $1.15 \pm 0.15$ & $0.7 \pm 0.2$ & $1.85 \pm 0.17$ & $2.0 \pm 0.2$ & $0.49 \pm 0.13$ & $<0.9$ & $-11.1 \pm 1.8$ & $-4.20 \pm 0.06$ & $1.14 \pm 0.02$ \\
\hline J0331-3042 & M9 & $<0.6$ & $1.45 \pm 0.14$ & $0.93 \pm 0.18$ & $1.9 \pm 0.2$ & $2.2 \pm 0.3$ & $0.58 \pm 0.09$ & $<0.9$ & $-7.9 \pm 0.5$ & $-4.72 \pm 0.06$ & $1.138 \pm 0.015$ \\
\hline J0339-3525 & M9 & $0.8 \pm 0.3$ & $1.19 \pm 0.16$ & $0.64 \pm 0.12$ & $1.27 \pm 0.15$ & $1.5 \pm 0.2$ & $0.79 \pm 0.14$ & $<0.9$ & $-0.9 \pm 0.2$ & $-5.70 \pm 0.06$ & $1.17 \pm 0.06$ \\
\hline J0351-0052 & M7.5 & $<1.2$ & $0.88 \pm 0.08$ & $0.68 \pm 0.10$ & $1.75 \pm 0.18$ & $1.9 \pm 0.3$ & $0.32 \pm 0.07$ & $<1.0$ & $-5.8 \pm 0.2$ & $-4.51 \pm 0.06$ & $1.20 \pm 0.03$ \\
\hline J0500+0330 & L4 & $<0.9$ & $3.8 \pm 0.5$ & $3.4 \pm 0.4$ & $1.18 \pm 0.15$ & $2.0 \pm 0.3$ & $2.5 \pm 0.2$ & $1.6 \pm 0.2$ & $>-1.1$ & $<-6.5$ & $\ldots$ \\
\hline J0517-3349 & M8 & $<0.8$ & $1.5 \pm 0.3$ & $1.14 \pm 0.14$ & $1.4 \pm 0.3$ & $1.7 \pm 0.4$ & $0.6 \pm 0.2$ & $<1.2$ & $-10.1 \pm 0.8$ & $-4.34 \pm 0.06$ & $0.863 \pm 0.015$ \\
\hline J0544-2433 & M9 & $<0.4$ & $1.25 \pm 0.14$ & $0.9 \pm 0.2$ & $1.46 \pm 0.13$ & $2.0 \pm 0.3$ & $0.52 \pm 0.13$ & $<0.8$ & $-3.6 \pm 0.3$ & $-5.03 \pm 0.06$ & $1.13 \pm 0.02$ \\
\hline J0615-0100 & $\mathrm{L} 1$ & $<0.3$ & $2.7 \pm 0.3$ & $2.2 \pm 0.2$ & $1.4 \pm 0.2$ & $2.0 \pm 0.3$ & $1.39 \pm 0.13$ & $1.05 \pm 0.12$ & $>-0.6$ & $<-6.5$ & $\ldots$ \\
\hline J0641-4322 & L3 & $<1.1$ & $3.0 \pm 0.4$ & $2.5 \pm 0.3$ & $1.8 \pm 0.3$ & $2.2 \pm 0.3$ & $1.50 \pm 0.13$ & $1.01 \pm 0.16$ & $>-0.8$ & $<-6.5$ & $\ldots$ \\
\hline J0652-2534 & M9 & $0.5 \pm 0.2$ & $1.10 \pm 0.16$ & $0.72 \pm 0.11$ & $1.26 \pm 0.14$ & $1.5 \pm 0.2$ & $0.63 \pm 0.13$ & $<1.0$ & $>-0.4$ & $<-6.6$ & $1.05 \pm 0.03$ \\
\hline J0707-4900 & M8.5 & $<0.7$ & $1.5 \pm 0.2$ & $1.03 \pm 0.13$ & $2.0 \pm 0.2$ & $2.1 \pm 0.3$ & $0.71 \pm 0.14$ & $<0.9$ & $-1.3 \pm 0.2$ & $-5.43 \pm 0.06$ & $1.01 \pm 0.02$ \\
\hline J0751-2530 & L1 & $<0.3$ & $2.8 \pm 0.3$ & $1.97 \pm 0.16$ & $1.70 \pm 0.15$ & $2.1 \pm 0.2$ & $1.34 \pm 0.12$ & $1.09 \pm 0.12$ & $>-0.3$ & $<-6.7$ & $\ldots$ \\
\hline J0812-2444 & $\mathrm{L} 2.5$ & $<0.4$ & $2.9 \pm 0.4$ & $2.11 \pm 0.17$ & $1.40 \pm 0.16$ & $1.9 \pm 0.2$ & $1.46 \pm 0.10$ & $1.09 \pm 0.12$ & $-1.9 \pm 0.3$ & $-5.53 \pm 0.06$ & $\ldots$ \\
\hline J0823-4912AB & L3 & $2.6 \pm 0.4$ & $2.6 \pm 0.3$ & $2.0 \pm 0.2$ & $0.99 \pm 0.14$ & $1.5 \pm 0.2$ & $1.58 \pm 0.12$ & $0.99 \pm 0.13$ & $>-0.8$ & $<-6.5$ & $\ldots$ \\
\hline J0830+0947 & M7.5 & $<0.4$ & $1.04 \pm 0.11$ & $0.9 \pm 0.2$ & $2.0 \pm 0.2$ & $2.3 \pm 0.3$ & $0.58 \pm 0.09$ & $<0.8$ & $-19.9 \pm 1.3$ & $-3.95 \pm 0.06$ & $1.038 \pm 0.013$ \\
\hline J0835-0819 & L6 & $<0.5$ & $3.6 \pm 0.5$ & $3.7 \pm 0.4$ & $1.23 \pm 0.09$ & $1.79 \pm 0.17$ & $2.8 \pm 0.2$ & $1.3 \pm 0.3$ & $>-0.5$ & $<-7.3$ & $\ldots$ \\
\hline J0847-1532 & $\mathrm{L} 2$ & $<0.3$ & $2.6 \pm 0.3$ & $2.1 \pm 0.2$ & $1.36 \pm 0.14$ & $1.74 \pm 0.17$ & $1.38 \pm 0.10$ & $1.02 \pm 0.08$ & $-1.02 \pm 0.18$ & $-5.74 \pm 0.06$ & $\ldots$ \\
\hline J0853-0329 & M9 & $<0.3$ & $1.02 \pm 0.09$ & $0.61 \pm 0.16$ & $1.51 \pm 0.11$ & $1.7 \pm 0.2$ & $0.66 \pm 0.11$ & $<0.8$ & $-8.8 \pm 0.2$ & $-4.60 \pm 0.06$ & $1.006 \pm 0.015$ \\
\hline J0902+0033 & M7 & $<0.6$ & $1.05 \pm 0.10$ & $0.77 \pm 0.18$ & $1.77 \pm 0.11$ & $2.2 \pm 0.3$ & $0.37 \pm 0.11$ & $<0.6$ & $-5.7 \pm 0.2$ & $-4.38 \pm 0.06$ & $1.087 \pm 0.007$ \\
\hline J0909-0658 & $\mathrm{L} 1$ & $<1.8$ & $2.0 \pm 0.3$ & $1.65 \pm 0.16$ & $1.32 \pm 0.16$ & $1.9 \pm 0.2$ & $1.08 \pm 0.08$ & $0.74 \pm 0.11$ & $-1.5 \pm 0.6$ & $-5.64 \pm 0.07$ & $\ldots$ \\
\hline J0921-2104 & L1 & $<0.3$ & $3.2 \pm 0.3$ & $2.4 \pm 0.2$ & $1.70 \pm 0.18$ & $2.1 \pm 0.2$ & $1.66 \pm 0.08$ & $1.36 \pm 0.12$ & $>-0.4$ & $<-6.8$ & $\ldots$ \\
\hline J0923+2340 & L1 & $<1.6$ & $2.7 \pm 0.3$ & $2.25 \pm 0.18$ & $1.8 \pm 0.2$ & $2.1 \pm 0.4$ & $1.53 \pm 0.09$ & $1.38 \pm 0.09$ & $-1.3 \pm 0.6$ & $-5.63 \pm 0.07$ & $\ldots$ \\
\hline J0949+0806 & M8.5 & $<0.7$ & $1.6 \pm 0.2$ & $1.08 \pm 0.12$ & $1.88 \pm 0.17$ & $2.2 \pm 0.4$ & $0.74 \pm 0.15$ & $<0.7$ & $-2.3 \pm 0.5$ & $-5.17 \pm 0.06$ & $1.013 \pm 0.011$ \\
\hline J1003-0105 & M8 & $<1.4$ & $1.4 \pm 0.2$ & $1.0 \pm 0.2$ & $1.87 \pm 0.14$ & $2.5 \pm 0.4$ & $0.74 \pm 0.12$ & $<0.5$ & $-7.8 \pm 0.7$ & $-4.50 \pm 0.06$ & $0.974 \pm 0.016$ \\
\hline J1004-3335 & $\mathrm{L} 4$ & $<1.6$ & $3.1 \pm 0.5$ & $3.4 \pm 0.3$ & $1.09 \pm 0.18$ & $1.7 \pm 0.3$ & $2.6 \pm 0.2$ & $1.9 \pm 0.2$ & $>-2$ & $<-6.4$ & $\ldots$ \\
\hline J1045-0149 & $\mathrm{L} 2$ & $<0.2$ & $2.5 \pm 0.3$ & $1.83 \pm 0.14$ & $1.38 \pm 0.17$ & $1.7 \pm 0.2$ & $1.18 \pm 0.09$ & $0.92 \pm 0.10$ & $-0.60 \pm 0.11$ & $-6.00 \pm 0.06$ & $\ldots$ \\
\hline J1048-3956 & M8 & $<0.3$ & $1.50 \pm 0.16$ & $1.26 \pm 0.15$ & $1.9 \pm 0.2$ & $2.1 \pm 0.3$ & $0.80 \pm 0.11$ & $<0.7$ & $-7.4 \pm 0.9$ & $-4.52 \pm 0.06$ & $0.947 \pm 0.004$ \\
\hline
\end{tabular}


Table 3-Continued

\begin{tabular}{|c|c|c|c|c|c|c|c|c|c|c|c|}
\hline Source & SpT & $\begin{array}{c}\mathrm{Li} \mathrm{I} \\
6710 \AA\end{array}$ & $\begin{array}{c}\mathrm{Rb} \mathrm{I} \\
7802 \AA\end{array}$ & $\begin{array}{c}\mathrm{Rb} \mathrm{I} \\
7950 \AA\end{array}$ & $\begin{array}{c}\mathrm{Na} \mathrm{I} \\
8186 \AA\end{array}$ & $\begin{array}{c}\mathrm{Na} \mathrm{I} \\
8197 \AA\end{array}$ & $\begin{array}{c}\text { Cs I } \\
8523 \AA\end{array}$ & $\begin{array}{c}\text { Cs I } \\
8946 \AA\end{array}$ & $\begin{array}{c}\mathrm{H} \alpha \\
6583 \AA\end{array}$ & $\log _{10} L_{H \alpha} / L_{b o l}$ & $\zeta$ \\
\hline $\mathrm{J} 1054+1214$ & M7.5 & $<0.5$ & $1.06 \pm 0.11$ & $0.96 \pm 0.14$ & $1.74 \pm 0.18$ & $2.0 \pm 0.3$ & $0.46 \pm 0.11$ & $<0.7$ & $-4.6 \pm 0.4$ & $-4.56 \pm 0.06$ & $1.014 \pm 0.008$ \\
\hline $\mathrm{J} 1055+0808$ & M9 & $<0.6$ & $1.21 \pm 0.13$ & $0.92 \pm 0.16$ & $1.69 \pm 0.16$ & $1.9 \pm 0.3$ & $0.58 \pm 0.18$ & $<0.6$ & $-1.3 \pm 0.3$ & $-5.56 \pm 0.06$ & $0.978 \pm 0.014$ \\
\hline $\mathrm{J} 1119+0828$ & M7 & $<4$ & $1.2 \pm 0.2$ & $0.7 \pm 0.2$ & $1.5 \pm 0.2$ & $2.0 \pm 0.4$ & $<0.4$ & $<0.8$ & $-1.8 \pm 0.9$ & $-4.93 \pm 0.06$ & $1.12 \pm 0.05$ \\
\hline J1121-1313AB & M8.5 & $<0.5$ & $1.29 \pm 0.15$ & $1.04 \pm 0.16$ & $1.9 \pm 0.2$ & $2.2 \pm 0.3$ & $0.75 \pm 0.13$ & $<0.6$ & $-9.4 \pm 0.4$ & $-4.52 \pm 0.06$ & $0.952 \pm 0.011$ \\
\hline $\mathrm{J} 1134+0022$ & M9 & $<0.8$ & $1.19 \pm 0.13$ & $0.70 \pm 0.13$ & $1.74 \pm 0.14$ & $1.9 \pm 0.2$ & $0.57 \pm 0.11$ & $<0.8$ & $-14.5 \pm 0.7$ & $-4.42 \pm 0.06$ & $1.136 \pm 0.015$ \\
\hline J1139-3159 & M9 & $0.6 \pm 0.3$ & $0.90 \pm 0.12$ & $<0.7$ & $0.97 \pm 0.08$ & $0.90 \pm 0.15$ & $<0.5$ & $<1.3$ & $-9.4 \pm 0.7$ & $-4.67 \pm 0.06$ & $1.32 \pm 0.02$ \\
\hline J1155-3727 & L3 & $<0.2$ & $2.9 \pm 0.3$ & $2.4 \pm 0.2$ & $1.65 \pm 0.15$ & $2.1 \pm 0.2$ & $1.88 \pm 0.16$ & $1.62 \pm 0.17$ & $-2.44 \pm 0.16$ & $-5.44 \pm 0.06$ & $\ldots$ \\
\hline $\mathrm{J} 1221+0257$ & L0 & $<0.5$ & $1.7 \pm 0.2$ & $1.27 \pm 0.14$ & $1.52 \pm 0.16$ & $1.8 \pm 0.3$ & $0.95 \pm 0.10$ & $0.7 \pm 0.2$ & $-3.00 \pm 0.16$ & $-5.22 \pm 0.06$ & $\ldots$ \\
\hline J1224-1238 & M9 & $<0.3$ & $1.34 \pm 0.16$ & $0.87 \pm 0.12$ & $1.7 \pm 0.2$ & $1.9 \pm 0.3$ & $0.47 \pm 0.09$ & $<0.8$ & $-4.5 \pm 0.3$ & $-4.95 \pm 0.06$ & $1.086 \pm 0.012$ \\
\hline $\mathrm{J} 1252+0252$ & M7 & $<1.3$ & $1.21 \pm 0.07$ & $0.97 \pm 0.09$ & $1.63 \pm 0.18$ & $2.3 \pm 0.3$ & $0.47 \pm 0.09$ & $<0.6$ & $-6.5 \pm 0.7$ & $-4.44 \pm 0.06$ & $1.07 \pm 0.03$ \\
\hline J1309-2330 & M8 & $<0.5$ & $1.5 \pm 0.2$ & $1.03 \pm 0.17$ & $1.87 \pm 0.15$ & $2.1 \pm 0.3$ & $0.59 \pm 0.10$ & $<1.0$ & $-4.06 \pm 0.14$ & $-4.82 \pm 0.06$ & $1.078 \pm 0.008$ \\
\hline J1315-2649AB & L5 & $<0.9$ & $3.8 \pm 0.7$ & $3.3 \pm 0.6$ & $1.13 \pm 0.13$ & $1.7 \pm 0.2$ & $3.5 \pm 0.4$ & $2.10 \pm 0.13$ & $-53 \pm 20$ & $-4.17 \pm 0.06$ & $\ldots$ \\
\hline J1332-0441 & M7.5 & $<0.4$ & $1.22 \pm 0.13$ & $0.83 \pm 0.18$ & $1.76 \pm 0.18$ & $2.0 \pm 0.3$ & $0.39 \pm 0.11$ & $<0.7$ & $-7.1 \pm 0.5$ & $-4.44 \pm 0.06$ & $1.062 \pm 0.008$ \\
\hline J1411-2119 & M9 & $0.68 \pm 0.11$ & $1.17 \pm 0.14$ & $0.7 \pm 0.2$ & $1.45 \pm 0.15$ & $1.6 \pm 0.2$ & $0.56 \pm 0.13$ & $<0.7$ & $-2.08 \pm 0.16$ & $-5.31 \pm 0.06$ & $1.123 \pm 0.009$ \\
\hline $\mathrm{J} 1422+2116$ & M7 & $<1.0$ & $1.34 \pm 0.11$ & $1.10 \pm 0.11$ & $1.6 \pm 0.2$ & $2.1 \pm 0.3$ & $0.61 \pm 0.11$ & $<0.6$ & $-9.9 \pm 0.2$ & $-4.18 \pm 0.06$ & $0.979 \pm 0.006$ \\
\hline $\mathrm{J} 1440+1339$ & M7 & $<0.5$ & $1.02 \pm 0.11$ & $0.72 \pm 0.15$ & $1.43 \pm 0.16$ & $1.9 \pm 0.3$ & $<0.3$ & $<1.0$ & $-7.4 \pm 0.2$ & $-4.23 \pm 0.06$ & $1.164 \pm 0.009$ \\
\hline J1456-2809 & M7 & $<0.5$ & $1.27 \pm 0.13$ & $0.90 \pm 0.13$ & $1.8 \pm 0.2$ & $2.1 \pm 0.3$ & $0.44 \pm 0.08$ & $<0.8$ & $-5.5 \pm 0.2$ & $-4.48 \pm 0.06$ & $1.030 \pm 0.003$ \\
\hline J1500-0039 & M6 & $<0.3$ & $0.66 \pm 0.05$ & $0.59 \pm 0.10$ & $1.51 \pm 0.18$ & $1.9 \pm 0.3$ & $<0.13$ & $<0.5$ & $-6.0 \pm 0.3$ & $-4.11 \pm 0.06$ & $1.037 \pm 0.004$ \\
\hline $\mathrm{J} 1501+2250$ & M9 & $<0.4$ & $1.28 \pm 0.14$ & $0.92 \pm 0.09$ & $1.1 \pm 0.2$ & $1.7 \pm 0.4$ & $0.83 \pm 0.11$ & $0.7 \pm 0.2$ & $-3.3 \pm 0.4$ & $-5.00 \pm 0.06$ & $0.913 \pm 0.015$ \\
\hline J1504-2355 & M7.5 & $<0.5$ & $1.18 \pm 0.14$ & $0.91 \pm 0.12$ & $1.8 \pm 0.2$ & $1.9 \pm 0.3$ & $0.52 \pm 0.10$ & $<0.7$ & $-4.9 \pm 0.4$ & $-4.59 \pm 0.06$ & $1.023 \pm 0.007$ \\
\hline $\mathrm{J} 1506+1321$ & L3 & $<0.3$ & $3.3 \pm 0.4$ & $2.9 \pm 0.3$ & $1.20 \pm 0.13$ & $1.79 \pm 0.17$ & $2.00 \pm 0.10$ & $1.5 \pm 0.2$ & $-0.5 \pm 0.3$ & $-6.11 \pm 0.06$ & $\ldots$ \\
\hline J1510-0241 & M9 & $<0.6$ & $1.31 \pm 0.13$ & $0.93 \pm 0.13$ & $1.9 \pm 0.2$ & $1.9 \pm 0.2$ & $0.74 \pm 0.09$ & $<0.7$ & $-14.7 \pm 1.4$ & $-4.38 \pm 0.06$ & $1.020 \pm 0.011$ \\
\hline J1510-2818 & M9 & $1.2 \pm 0.2$ & $1.17 \pm 0.13$ & $<0.6$ & $1.38 \pm 0.18$ & $1.42 \pm 0.18$ & $0.62 \pm 0.16$ & $<1.2$ & $-6.1 \pm 0.2$ & $-4.85 \pm 0.06$ & $1.139 \pm 0.016$ \\
\hline J1534-1418 & M8 & $<0.5$ & $2.2 \pm 0.3$ & $1.7 \pm 0.3$ & $2.3 \pm 0.4$ & $2.4 \pm 0.3$ & $0.77 \pm 0.12$ & $<0.8$ & $-1.07 \pm 0.15$ & $-5.20 \pm 0.06$ & $0.982 \pm 0.005$ \\
\hline J1539-0520 & $\mathrm{L} 4$ & $<0.6$ & $3.7 \pm 0.4$ & $3.6 \pm 0.4$ & $1.21 \pm 0.18$ & $2.0 \pm 0.3$ & $2.6 \pm 0.3$ & $2.1 \pm 0.2$ & $>-1.4$ & $<-6.5$ & $\ldots$ \\
\hline $\mathrm{J} 1550+1455 \mathrm{AB}$ & L3.5 & $<2$ & $2.9 \pm 0.3$ & $2.3 \pm 0.3$ & $1.11 \pm 0.11$ & $1.6 \pm 0.2$ & $1.62 \pm 0.07$ & $1.2 \pm 0.2$ & $>-1.8$ & $<-6.4$ & $\ldots$ \\
\hline $\mathrm{J} 1553+1400$ & M9 & $<0.4$ & $1.16 \pm 0.10$ & $0.78 \pm 0.12$ & $1.8 \pm 0.3$ & $1.7 \pm 0.2$ & $<0.5$ & $<0.8$ & $-5.2 \pm 0.3$ & $-4.85 \pm 0.06$ & $1.18 \pm 0.03$ \\
\hline J1555-0956 & $\mathrm{L} 1$ & $<0.3$ & $2.9 \pm 0.3$ & $2.4 \pm 0.2$ & $1.8 \pm 0.2$ & $2.2 \pm 0.3$ & $1.64 \pm 0.13$ & $1.35 \pm 0.08$ & $-0.6 \pm 0.2$ & $-6.00 \pm 0.06$ & $\ldots$ \\
\hline J1607-0442 & M9 & $<0.5$ & $1.4 \pm 0.2$ & $0.8 \pm 0.2$ & $1.70 \pm 0.14$ & $1.9 \pm 0.2$ & $0.52 \pm 0.09$ & $<0.9$ & $-10.8 \pm 0.6$ & $-4.58 \pm 0.06$ & $1.119 \pm 0.011$ \\
\hline $\mathrm{J} 1615+0546$ & M9 & $<0.5$ & $1.34 \pm 0.17$ & $0.94 \pm 0.15$ & $1.47 \pm 0.16$ & $1.7 \pm 0.2$ & $0.76 \pm 0.12$ & $<0.8$ & $>-0.4$ & $<-6.7$ & $0.982 \pm 0.014$ \\
\hline J1645-1319 & L1.5 & $<0.2$ & $2.9 \pm 0.3$ & $2.16 \pm 0.16$ & $1.51 \pm 0.14$ & $1.9 \pm 0.2$ & $1.49 \pm 0.08$ & $1.27 \pm 0.08$ & $-2.18 \pm 0.10$ & $-5.41 \pm 0.06$ & $\ldots$ \\
\hline J1705-0516 & L0.5 & $<0.6$ & $2.7 \pm 0.3$ & $1.90 \pm 0.12$ & $1.2 \pm 0.2$ & $1.7 \pm 0.2$ & $1.23 \pm 0.09$ & $1.12 \pm 0.11$ & $>-1.0$ & $<-6.4$ & $\cdots$ \\
\hline J1707-0558AB & L0 & $<0.2$ & $1.25 \pm 0.12$ & $0.80 \pm 0.12$ & $1.44 \pm 0.12$ & $1.7 \pm 0.2$ & $0.67 \pm 0.10$ & $<0.8$ & $-0.59 \pm 0.11$ & $-6.00 \pm 0.06$ & $\ldots$ \\
\hline J1733-1654 & L0.5 & $<0.6$ & $3.0 \pm 0.3$ & $2.6 \pm 0.2$ & $1.7 \pm 0.2$ & $2.1 \pm 0.3$ & $1.68 \pm 0.13$ & $1.57 \pm 0.09$ & $>-1.3$ & $<-6.5$ & $\ldots$ \\
\hline
\end{tabular}


Table 3-Continued

\begin{tabular}{|c|c|c|c|c|c|c|c|c|c|c|c|}
\hline Source & $\mathrm{SpT}$ & $\begin{array}{c}\mathrm{Li} \mathrm{I} \\
6710 \AA\end{array}$ & $\begin{array}{c}\mathrm{Rb} \mathrm{I} \\
7802 \AA\end{array}$ & $\begin{array}{c}\mathrm{Rb} I \\
7950 \AA\end{array}$ & $\begin{array}{c}\mathrm{Na} \text { I } \\
8186 \AA\end{array}$ & $\begin{array}{c}\mathrm{Na} I \\
8197 \AA\end{array}$ & $\begin{array}{c}\text { Cs I } \\
8523 \AA\end{array}$ & $\begin{array}{c}\text { Cs I } \\
8946 \AA\end{array}$ & $\begin{array}{c}\mathrm{H} \alpha \\
6583 \AA\end{array}$ & $\log _{10} L_{H \alpha} / L_{b o l}$ & $\zeta$ \\
\hline J1745-1640 & $\mathrm{L} 1.5$ & $<0.4$ & $2.9 \pm 0.4$ & $2.3 \pm 0.2$ & $1.50 \pm 0.14$ & $2.1 \pm 0.3$ & $1.59 \pm 0.12$ & $1.32 \pm 0.06$ & $-1.5 \pm 0.3$ & $-5.10 \pm 0.06$ & $\ldots$ \\
\hline J1750-0016 & L5.5 & $<0.2$ & $4.3 \pm 0.4$ & $4.3 \pm 0.5$ & $1.16 \pm 0.10$ & $1.9 \pm 0.2$ & $3.3 \pm 0.3$ & $2.14 \pm 0.16$ & $>-0.4$ & $<-7.1$ & $\ldots$ \\
\hline J1845-6357 & M8.5 & $<0.3$ & $1.7 \pm 0.2$ & $1.53 \pm 0.12$ & $1.8 \pm 0.2$ & $2.1 \pm 0.3$ & $0.94 \pm 0.13$ & $<1.2$ & $-3.2 \pm 0.2$ & $-5.00 \pm 0.06$ & $0.953 \pm 0.003$ \\
\hline J2000-7523 & M9 & $<0.8$ & $0.9 \pm 0.3$ & $0.67 \pm 0.15$ & $0.86 \pm 0.11$ & $1.1 \pm 0.2$ & $<0.7$ & $<0.9$ & $-1.6 \pm 0.5$ & $-5.53 \pm 0.06$ & $1.40 \pm 0.03$ \\
\hline $\mathrm{J} 2036+1051$ & L3 & $<1.0$ & $3.4 \pm 0.6$ & $2.1 \pm 0.4$ & $1.2 \pm 0.3$ & $1.7 \pm 0.3$ & $1.79 \pm 0.17$ & $1.00 \pm 0.14$ & $-11 \pm 3$ & $-4.87 \pm 0.06$ & $\ldots$ \\
\hline J2037-1137 & M8 & $<0.8$ & $1.24 \pm 0.13$ & $0.83 \pm 0.16$ & $1.75 \pm 0.14$ & $2.0 \pm 0.2$ & $0.38 \pm 0.12$ & $<0.9$ & $>-0.3$ & $<-6.7$ & $1.144 \pm 0.010$ \\
\hline $\mathrm{J} 2045-6332$ & M9 & $1.02 \pm 0.10$ & $1.17 \pm 0.14$ & $0.63 \pm 0.15$ & $1.23 \pm 0.10$ & $1.29 \pm 0.16$ & $0.68 \pm 0.13$ & $<1.0$ & $-1.28 \pm 0.13$ & $-5.52 \pm 0.06$ & $1.17 \pm 0.02$ \\
\hline J2104-1037 & L3 & $<0.5$ & $3.0 \pm 0.4$ & $2.6 \pm 0.3$ & $1.44 \pm 0.17$ & $1.8 \pm 0.2$ & $1.69 \pm 0.13$ & $1.24 \pm 0.13$ & $>-0.5$ & $<-6.9$ & $\ldots$ \\
\hline $\mathrm{J} 2120+1021$ & M8 & $<0.5$ & $1.6 \pm 0.2$ & $1.43 \pm 0.10$ & $2.0 \pm 0.2$ & $2.2 \pm 0.3$ & $0.91 \pm 0.14$ & $0.6 \pm 0.2$ & $-4.5 \pm 0.4$ & $-4.81 \pm 0.06$ & $0.970 \pm 0.009$ \\
\hline J2200-3038AB & M8 & $<0.4$ & $1.34 \pm 0.11$ & $1.24 \pm 0.12$ & $1.86 \pm 0.17$ & $2.2 \pm 0.3$ & $1.02 \pm 0.17$ & $<1.1$ & $-3.6 \pm 0.2$ & $-4.89 \pm 0.06$ & $1.054 \pm 0.014$ \\
\hline $\mathrm{J} 2226-7503$ & M8 & $<0.8$ & $1.45 \pm 0.15$ & $1.05 \pm 0.12$ & $1.74 \pm 0.15$ & $2.0 \pm 0.3$ & $0.68 \pm 0.08$ & $<0.6$ & $-6.1 \pm 0.3$ & $-4.56 \pm 0.06$ & $1.030 \pm 0.011$ \\
\hline $\mathrm{J} 2306-0502$ & M8 & $<0.6$ & $1.40 \pm 0.14$ & $0.9 \pm 0.2$ & $1.87 \pm 0.17$ & $1.9 \pm 0.2$ & $0.45 \pm 0.10$ & $<1.0$ & $-4.86 \pm 0.18$ & $-4.73 \pm 0.06$ & $1.139 \pm 0.012$ \\
\hline $\mathrm{J} 2331-2749$ & M7.5 & $<0.6$ & $1.67 \pm 0.16$ & $1.53 \pm 0.15$ & $2.2 \pm 0.2$ & $2.4 \pm 0.4$ & $0.69 \pm 0.11$ & $0.58 \pm 0.18$ & $-2.25 \pm 0.13$ & $-5.01 \pm 0.06$ & $0.960 \pm 0.006$ \\
\hline $\mathrm{J} 2337-0838$ & M8 & $<0.5$ & $1.14 \pm 0.13$ & $0.80 \pm 0.16$ & $1.69 \pm 0.14$ & $2.0 \pm 0.3$ & $0.44 \pm 0.08$ & $<0.6$ & $-9.9 \pm 0.4$ & $-4.40 \pm 0.06$ & $1.072 \pm 0.009$ \\
\hline $\mathrm{J} 2346+1129$ & M8 & $<0.4$ & $1.30 \pm 0.14$ & $0.94 \pm 0.15$ & $1.75 \pm 0.18$ & $2.0 \pm 0.2$ & $0.75 \pm 0.10$ & $<0.8$ & $-6.7 \pm 0.5$ & $-4.60 \pm 0.06$ & $1.06 \pm 0.02$ \\
\hline $\mathrm{J} 2349+1224$ & M8 & $<0.3$ & $1.45 \pm 0.18$ & $1.1 \pm 0.2$ & $1.8 \pm 0.2$ & $2.1 \pm 0.3$ & $0.55 \pm 0.17$ & $<0.8$ & $-3.85 \pm 0.10$ & $-4.84 \pm 0.06$ & $1.129 \pm 0.011$ \\
\hline $\mathrm{J} 2351-2537$ & M8 & $<0.4$ & $1.9 \pm 0.2$ & $1.47 \pm 0.15$ & $1.8 \pm 0.2$ & $2.0 \pm 0.2$ & $1.1 \pm 0.2$ & $0.9 \pm 0.2$ & $-10.5 \pm 1.1$ & $-4.36 \pm 0.06$ & $0.83 \pm 0.02$ \\
\hline
\end{tabular}


Table 4. Mean Values of Gravity-Sensitive Indices

\begin{tabular}{lccccc}
\hline \hline SpT & $\#$ & Na-a + Na-b & VO7900 & TiO-b & CrH-a + FeH-a \\
\hline M7 & 12 & $2.84 \pm 0.16$ & $1.23 \pm 0.11$ & $1.87 \pm 0.11$ & $2.17 \pm 0.10$ \\
M8 & 20 & $2.83 \pm 0.15$ & $1.24 \pm 0.08$ & $2.06 \pm 0.10$ & $2.25 \pm 0.13$ \\
M9 & 13 & $2.68 \pm 0.14$ & $1.10 \pm 0.06$ & $2.04 \pm 0.11$ & $2.18 \pm 0.12$ \\
L0 & 4 & $2.47 \pm 0.09$ & $1.04 \pm 0.14$ & $1.5 \pm 0.2$ & $2.7 \pm 0.3$ \\
L1 & 8 & $2.63 \pm 0.16$ & $1.08 \pm 0.09$ & $1.46 \pm 0.11$ & $2.79 \pm 0.13$ \\
L2 & 3 & $2.48 \pm 0.12$ & $1.13 \pm 0.06$ & $1.33 \pm 0.04$ & $2.81 \pm 0.08$ \\
L3 & 6 & $2.43 \pm 0.08$ & $1.28 \pm 0.13$ & $1.21 \pm 0.09$ & $3.02 \pm 0.17$ \\
L4 & 3 & $2.441 \pm 0.018$ & $1.37 \pm 0.09$ & $1.20 \pm 0.08$ & $3.40 \pm 0.08$ \\
L5 & 2 & $2.34 \pm 0.02$ & $1.86 \pm 0.04$ & $1.063 \pm 0.005$ & $3.4 \pm 0.2$ \\
\hline
\end{tabular}


Table 5. Low Surface Gravity Dwarfs

\begin{tabular}{|c|c|c|c|c|c|c|c|c|c|c|c|c|}
\hline \multirow[b]{2}{*}{ Source } & \multirow[b]{2}{*}{$\mathrm{SpT}$} & \multicolumn{2}{|c|}{$\mathrm{Na}-\mathrm{a}+\mathrm{Na}-\mathrm{b}$} & \multicolumn{2}{|c|}{ VO7900 } & \multicolumn{2}{|c|}{ TiO-b } & \multicolumn{2}{|c|}{$\mathrm{CrH}-\mathrm{a}+\mathrm{FeH}-\mathrm{a}$} & \multicolumn{3}{|c|}{ Prior } \\
\hline & & Value & Low g? & Value & Low g? & Value & Low g? & Value & Low g? & Li I? & YMG & Ref \\
\hline \multicolumn{13}{|c|}{ Strong Signatures of Low Surface Gravity } \\
\hline J0909-0658 & L1 & $2.28 \pm 0.03$ & Y? & $0.86 \pm 0.010$ & $\mathrm{Y} ?$ & $1.52 \pm 0.02$ & $\mathrm{~N}$ & $2.50 \pm 0.02$ & $\mathrm{Y} ?$ & $\mathrm{~N}$ & & \\
\hline J1139-3159 & $\mathrm{M} 9 \gamma$ & $2.151 \pm 0.012$ & Y & $0.94 \pm 0.004$ & $\mathrm{Y} ?$ & $2.316 \pm 0.017$ & $\mathrm{Y} ?$ & $1.845 \pm 0.007$ & $\mathrm{Y}$ & Y & TWA & 1 \\
\hline J1510-2818 & M9 & $2.367 \pm 0.014$ & $\mathrm{Y} ?$ & $1.002 \pm 0.004$ & $\mathrm{Y} ?$ & $2.358 \pm 0.016$ & $\mathrm{Y}$ & $2.021 \pm 0.007$ & $\mathrm{Y} ?$ & Y & & \\
\hline J2000-7523 & M9 & $2.183 \pm 0.013$ & $\mathrm{Y}$ & $0.94 \pm 0.005$ & $\mathrm{Y} ?$ & $2.42 \pm 0.02$ & $\mathrm{Y}$ & $1.920 \pm 0.008$ & $\mathrm{Y} ?$ & $\mathrm{~N}$ & $\beta \mathrm{PMG}$ & 2,3 \\
\hline J2045-6332 & M9 & $2.222 \pm 0.009$ & $\mathrm{Y}$ & $1.044 \pm 0.004$ & $\mathrm{Y} ?$ & $2.274 \pm 0.012$ & $\mathrm{Y} ?$ & $2.073 \pm 0.005$ & $\mathrm{Y} ?$ & $\mathrm{Y}$ & $\beta \mathrm{PMG}$ & 3 \\
\hline \multicolumn{13}{|c|}{ Weak Signatures of Low Surface Gravity } \\
\hline J0041-5621AB & M8 & $2.385 \pm 0.011$ & Y? & $1.282 \pm 0.005$ & $\mathrm{~N}$ & $2.216 \pm 0.012$ & $\mathrm{Y} ?$ & $2.075 \pm 0.006$ & $\mathrm{~N}$ & $\bar{Y}$ & THA & 2,3 \\
\hline J0123-6921 & M9 & $2.408 \pm 0.014$ & $\mathrm{Y} ?$ & $1.264 \pm 0.006$ & $\mathrm{~N}$ & $2.049 \pm 0.014$ & $\mathrm{~N}$ & $2.000 \pm 0.006$ & $Y ?$ & $\mathrm{Y}$ & THA & 3 \\
\hline J0339-3525 & M9 & $2.407 \pm 0.013$ & $\mathrm{Y} ?$ & $1.054 \pm 0.006$ & $\mathrm{Y} ?$ & $2.127 \pm 0.015$ & $\mathrm{~N}$ & $2.152 \pm 0.007$ & $\mathrm{~N}$ & $\mathrm{Y} ?$ & CAS,ARG & 3,4 \\
\hline J0652-2534 & M9 & $2.419 \pm 0.011$ & $\mathrm{Y} ?$ & $1.021 \pm 0.004$ & $\mathrm{Y} ?$ & $2.057 \pm 0.013$ & $\mathrm{~N}$ & $2.172 \pm 0.006$ & $\mathrm{~N}$ & $\mathrm{Y}$ & & \\
\hline J0823-4912 & L3 & $2.29 \pm 0.02$ & $\mathrm{Y} ?$ & $1.110 \pm 0.007$ & $\mathrm{Y} ?$ & $1.240 \pm 0.009$ & $\mathrm{~N}$ & $2.836 \pm 0.013$ & $\mathrm{~N}$ & Y & & \\
\hline J1411-2119 & M9 & $2.478 \pm 0.011$ & $\mathrm{Y} ?$ & $1.111 \pm 0.004$ & $\mathrm{~N}$ & $2.137 \pm 0.012$ & $\mathrm{Y} ?$ & $2.033 \pm 0.005$ & $\mathrm{Y} ?$ & Y & & \\
\hline
\end{tabular}

References. - (1) Gizis (2002); (2) Gálvez-Ortiz et al. (2010); (3) Gagné et al. (2014); (4) Ribas (2003). 
Table 6. Radial Velocity Measurements

\begin{tabular}{|c|c|c|c|c|c|c|c|}
\hline Source & $\mathrm{SpT}$ & $\begin{array}{c}\text { Line } \\
\text { Centers } \\
\left(\mathrm{km} \mathrm{s}^{-1}\right)\end{array}$ & $\begin{array}{l}\text { SDSS } \\
\text { Templates } \\
\left(\mathrm{km} \mathrm{s}^{-1}\right)\end{array}$ & $\begin{array}{c}\text { MagE } \\
\text { Standards } \\
\left(\mathrm{km} \mathrm{s}^{-1}\right)\end{array}$ & $\begin{array}{l}\text { Adopted } \\
\text { Value } \\
\left(\mathrm{km} \mathrm{s}^{-1}\right)\end{array}$ & $\begin{array}{l}\text { Previous } \\
\text { Measures } \\
\left(\mathrm{km} \mathrm{s}^{-1}\right)\end{array}$ & Ref. \\
\hline J0004-2058 & M9 & $-33 \pm 5$ & $-26 \pm 4$ & $-32.9 \pm 0.9$ & $-32.9 \pm 2.2$ & $\ldots$ & \\
\hline J0024-0158 & M9.5 & $9 \pm 7$ & $18 \pm 5$ & $4.3 \pm 1.6$ & $4.3 \pm 2.6$ & $10 \pm 3$ & 5 \\
\hline J0041-5621AB & M8 & $7 \pm 4$ & $13 \pm 5$ & $5.0 \pm 0.8$ & $5.0 \pm 2.2$ & $2.4 \pm 1.0,7 \pm 3$ & 3,5 \\
\hline J0102-3737 & M9 & $-2 \pm 2$ & $4.5 \pm 1.2$ & $-4.9 \pm 1.4$ & $-4.9 \pm 2.4$ & $\ldots$ & \\
\hline J0109-0343 & M8 & $-6 \pm 5$ & $2 \pm 7$ & $-6.5 \pm 0.9$ & $-6.5 \pm 2.2$ & $-10.4 \pm 0.6,-7 \pm 3$ & 3,5 \\
\hline J0123-6921 & M9 & $13 \pm 5$ & $16 \pm 3$ & $9.6 \pm 1.1$ & $9.6 \pm 2.3$ & $7.5 \pm 0.3,11 \pm 3$ & 3,5 \\
\hline J0148-3024 & M7.5 & $15 \pm 11$ & $18 \pm 4$ & $11.7 \pm 1.4$ & $11.7 \pm 2.4$ & $10 \pm 3$ & 5 \\
\hline J0248-1651 & M8 & $5 \pm 5$ & $8 \pm 6$ & $2.9 \pm 0.8$ & $2.9 \pm 2.2$ & $4 \pm 3$ & 5 \\
\hline J0331-3042 & M9 & $21 \pm 5$ & $27.1 \pm 1.4$ & $19.2 \pm 0.9$ & $19.2 \pm 2.2$ & $23 \pm 3$ & 5 \\
\hline J0339-3525 & M9 & $8 \pm 8$ & $18 \pm 8$ & $5.8 \pm 1.3$ & $5.8 \pm 2.4$ & $6 \pm 3$ & 4 \\
\hline J0351-0052 & M7.5 & $-13 \pm 6$ & $-8 \pm 3$ & $-11.4 \pm 1.0$ & $-11.4 \pm 2.2$ & $-15 \pm 3$ & 5 \\
\hline $\mathrm{J} 0500+0330^{\mathrm{a}}$ & L4 & $9 \pm 5$ & $\ldots$ & $5.1 \pm 1.8$ & $5.1 \pm 2.7$ & $15.94 \pm 0.16$ & 1 \\
\hline $\mathrm{J} 0517-3349^{\mathrm{a}}$ & M8 & $-36 \pm 7$ & $-26 \pm 6$ & $-38.8 \pm 1.0$ & $-38.8 \pm 2.2$ & $31 \pm 3$ & 5 \\
\hline J0544-2433 & M9 & $24 \pm 6$ & $28 \pm 3$ & $21.1 \pm 0.9$ & $21.1 \pm 2.2$ & $21 \pm 3$ & 5 \\
\hline J0615-0100 & L1 & $-23 \pm 7$ & $\ldots$ & $-21.0 \pm 1.0$ & $-21.0 \pm 2.2$ & $\ldots$ & \\
\hline J0641-4322 & L3 & $70 \pm 4$ & $\ldots$ & $74.4 \pm 1.4$ & $74.4 \pm 2.4$ & $\cdots$ & \\
\hline J0652-2534 & M9 & $17 \pm 6$ & $13 \pm 12$ & $11.8 \pm 1.2$ & $11.8 \pm 2.3$ & $\ldots$ & \\
\hline J0707-4900 & M8.5 & $117 \pm 10$ & $115 \pm 18$ & $113.4 \pm 0.8$ & $113.4 \pm 2.2$ & $116.4 \pm 1.5$ & 8 \\
\hline J0751-2530 & L1 & $35 \pm 6$ & $\ldots$ & $32.4 \pm 1.1$ & $32.4 \pm 2.3$ & $\ldots$ & \\
\hline J0812-2444 & $\mathrm{L} 2.5$ & $-6 \pm 9$ & $\cdots$ & $-3.3 \pm 1.0$ & $-3.3 \pm 2.2$ & $\cdots$ & \\
\hline J0823-4912 & L3 & $13 \pm 7$ & $\ldots$ & $12.5 \pm 1.3$ & $12.5 \pm 2.4$ & $\ldots$ & \\
\hline J0830+0947 & M7.5 & $45 \pm 6$ & $49 \pm 2$ & $41.2 \pm 0.9$ & $41.2 \pm 2.2$ & $\ldots$ & \\
\hline J0835-0819 & L6 & $37 \pm 6$ & $\ldots$ & $40.3 \pm 2.0$ & $40.3 \pm 2.8$ & $29.89 \pm 0.06,27 \pm 2$ & 1,7 \\
\hline J0847-1532 & L2 & $-1 \pm 8$ & $\ldots$ & $-0.8 \pm 0.6$ & $-0.8 \pm 2.1$ & $2.02 \pm 0.10$ & 1 \\
\hline J0853-0329 & M9 & $9 \pm 5$ & $16 \pm 5$ & $6.7 \pm 0.9$ & $6.7 \pm 2.2$ & $6 \pm 3,9.5 \pm 1.0$ & 5,4 \\
\hline J0902+0033 & M7 & $46 \pm 5$ & $46.7 \pm 0.6$ & $41.2 \pm 0.8$ & $41.2 \pm 2.2$ & $48 \pm 5$ & 9 \\
\hline J0909-0658 & $\mathrm{L} 1$ & $34 \pm 5$ & $34 \pm 13$ & $28.6 \pm 1.1$ & $28.6 \pm 2.3$ & $27 \pm 3$ & 4 \\
\hline J0921-2104 & L1 & $81 \pm 8$ & $\ldots$ & $80.5 \pm 1.3$ & $80.5 \pm 2.4$ & $80.54 \pm 0.11,80.0 \pm 1.1$ & 1,7 \\
\hline $\mathrm{J} 0923+2340$ & L1 & $-39 \pm 4$ & $\cdots$ & $-31.9 \pm 0.5$ & $-31.9 \pm 2.1$ & $-30 \pm 4$ & 6 \\
\hline J0949+0806 & M8.5 & $17 \pm 7$ & $25 \pm 3$ & $16.2 \pm 1.0$ & $16.2 \pm 2.2$ & $\ldots$ & \\
\hline J1003-0105 & M8 & $22 \pm 6$ & $32 \pm 4$ & $22.3 \pm 1.1$ & $22.3 \pm 2.3$ & $35 \pm 5$ & 9 \\
\hline J1004-3335 & L4 & $6 \pm 8$ & $\ldots$ & $-7.8 \pm 1.0$ & $-7.8 \pm 2.2$ & $\ldots$ & \\
\hline J1045-0149 & L2 & $10 \pm 8$ & $\ldots$ & $10.0 \pm 1.5$ & $10.0 \pm 2.5$ & $6.31 \pm 0.10,7.0 \pm 1.1$ & 1,7 \\
\hline J1048-3956 & M8 & $-14 \pm 9$ & $-2 \pm 7$ & $-10.8 \pm 1.3$ & $-10.8 \pm 2.4$ & $-13 \pm 3$ & 5 \\
\hline $\mathrm{J} 1054+1214$ & M7.5 & $-6 \pm 5$ & $-2.7 \pm 1.7$ & $-7.3 \pm 0.9$ & $-7.3 \pm 2.2$ & $\ldots$ & \\
\hline $\mathrm{J} 1055+0808$ & M9 & $5 \pm 10$ & $8 \pm 10$ & $5.0 \pm 0.9$ & $5.0 \pm 2.2$ & $19 \pm 5$ & 9 \\
\hline $\mathrm{J} 1119+0828$ & M7 & $-50 \pm 11$ & $-42 \pm 7$ & $-45.1 \pm 0.8$ & $-45.1 \pm 2.2$ & $-39 \pm 5$ & 9 \\
\hline J1121-1313AB & M8.5 & $33 \pm 6$ & $45.1 \pm 1.5$ & $33.9 \pm 0.6$ & $33.9 \pm 2.1$ & $32 \pm 3$ & 5 \\
\hline $\mathrm{J} 1134+0022$ & M9 & $34 \pm 5$ & $43 \pm 4$ & $33.2 \pm 0.9$ & $33.2 \pm 2.2$ & $\ldots$ & \\
\hline J1139-3159 & M9 & $8 \pm 7$ & $16 \pm 12$ & $7.0 \pm 1.1$ & $7.0 \pm 2.3$ & $12 \pm 2$ & 4 \\
\hline $\mathrm{J} 1155-3727$ & L3 & $48 \pm 8$ & $\ldots$ & $46.4 \pm 1.1$ & $46.4 \pm 2.3$ & $45.47 \pm 0.11,45.0 \pm 1.1$ & 1,7 \\
\hline $\mathrm{J} 1221+0257$ & L0 & $-6 \pm 6$ & $-6 \pm 4$ & $-8.0 \pm 1.7$ & $-8.0 \pm 2.6$ & $-8.79 \pm 0.14,-9.0 \pm 1.4$ & 1,7 \\
\hline $\mathrm{J} 1224-1238$ & M9 & $-1 \pm 7$ & $6 \pm 4$ & $-2.2 \pm 1.0$ & $-2.2 \pm 2.2$ & $-6 \pm 3$ & 5 \\
\hline $\mathrm{J} 1252+0252$ & M7 & $23 \pm 10$ & $31 \pm 5$ & $21.2 \pm 1.3$ & $21.2 \pm 2.4$ & $30 \pm 5$ & 9 \\
\hline
\end{tabular}


Table 6-Continued

\begin{tabular}{|c|c|c|c|c|c|c|c|}
\hline Source & $\mathrm{SpT}$ & $\begin{array}{c}\text { Line } \\
\text { Centers } \\
\left(\mathrm{km} \mathrm{s}^{-1}\right)\end{array}$ & \begin{tabular}{c}
\multicolumn{1}{c}{ SDSS } \\
Templates \\
$\left(\mathrm{km} \mathrm{s}^{-1}\right)$
\end{tabular} & $\begin{array}{c}\text { MagE } \\
\text { Standards } \\
\left(\mathrm{km} \mathrm{s}^{-1}\right)\end{array}$ & $\begin{array}{c}\text { Adopted } \\
\text { Value } \\
\left(\mathrm{km} \mathrm{s}^{-1}\right)\end{array}$ & $\begin{array}{l}\text { Previous } \\
\text { Measures } \\
\left(\mathrm{km} \mathrm{s}^{-1}\right)\end{array}$ & Ref. \\
\hline J1309-2330 & M8 & $19 \pm 6$ & $23 \pm 3$ & $14.6 \pm 0.8$ & $14.6 \pm 2.2$ & $19 \pm 3$ & 5 \\
\hline J1315-2649AB & L5 & $-6 \pm 10$ & $\ldots$ & $-7.5 \pm 1.9$ & $-7.5 \pm 2.8$ & $\ldots$ & \\
\hline J1332-0441 & M7.5 & $-11 \pm 5$ & $-7.4 \pm 1.9$ & $-12.3 \pm 0.9$ & $-12.3 \pm 2.2$ & $-17 \pm 3$ & 5 \\
\hline J1411-2119 & M9 & $4 \pm 14$ & $6 \pm 11$ & $-0.9 \pm 1.5$ & $-0.9 \pm 2.5$ & $-1 \pm 3$ & 5 \\
\hline $\mathrm{J} 1422+2116$ & M7 & $-4 \pm 7$ & $3 \pm 5$ & $-4.2 \pm 0.9$ & $-4.2 \pm 2.2$ & $\cdots$ & \\
\hline $\mathrm{J} 1440+1339$ & M7 & $-4 \pm 6$ & $-1 \pm 4$ & $-5.1 \pm 1.2$ & $-5.1 \pm 2.3$ & $-5 \pm 3$ & 5 \\
\hline J1456-2809 & M7 & $1 \pm 5$ & $7 \pm 5$ & $0.9 \pm 0.9$ & $0.9 \pm 2.2$ & $1 \pm 3$ & 5 \\
\hline J1500-0039 & M6 & $-13 \pm 8$ & $-10 \pm 5$ & $-13.3 \pm 1.0$ & $-13.3 \pm 2.2$ & $-17 \pm 5$ & 2 \\
\hline $\mathrm{J} 1501+2250$ & M9 & $2 \pm 7$ & $20 \pm 4$ & $5.9 \pm 1.3$ & $5.9 \pm 2.4$ & $\ldots$ & \\
\hline J1504-2355 & M7.5 & $-27 \pm 9$ & $-23.1 \pm 1.6$ & $-28.6 \pm 1.0$ & $-28.6 \pm 2.2$ & $\ldots$ & \\
\hline $\mathrm{J} 1506+1321$ & L3 & $0 \pm 7$ & $\ldots$ & $1.1 \pm 1.6$ & $1.1 \pm 2.6$ & $-0.68 \pm 0.11,-0.9 \pm 1.2$ & 1,7 \\
\hline J1510-0241 & M9 & $-42 \pm 6$ & $\ldots$ & $-41.4 \pm 1.1$ & $-41.4 \pm 2.3$ & $\ldots$ & \\
\hline J1510-2818 & M9 & $-14 \pm 5$ & $-32 \pm 3$ & $-13.3 \pm 1.1$ & $-13.3 \pm 2.3$ & $\ldots$ & \\
\hline J1534-1418 & M8 & $-65 \pm 11$ & $-69 \pm 15$ & $-70.7 \pm 1.1$ & $-70.7 \pm 2.3$ & $-76 \pm 3$ & 5 \\
\hline J1539-0520 & $\mathrm{L} 4$ & $26 \pm 7$ & $\ldots$ & $26.6 \pm 3.8$ & $26.6 \pm 4.3$ & $27.3 \pm 0.2$ & 1 \\
\hline $\mathrm{J} 1550+1455 \mathrm{AB}$ & L3.5 & $13 \pm 6$ & $\ldots$ & $12.9 \pm 0.6$ & $12.9 \pm 2.1$ & $\ldots$ & \\
\hline $\mathrm{J} 1553+1400$ & M9 & $-51 \pm 9$ & $-46 \pm 3$ & $-53.3 \pm 1.1$ & $-53.3 \pm 2.3$ & $\ldots$ & \\
\hline J1555-0956 & $\mathrm{L} 1$ & $14 \pm 8$ & $\cdots$ & $14.9 \pm 1.0$ & $14.9 \pm 2.2$ & $14.84 \pm 0.10,14.5 \pm 1.1$ & 1,7 \\
\hline J1607-0442 & M9 & $15 \pm 9$ & $19 \pm 4$ & $10.8 \pm 0.9$ & $10.8 \pm 2.2$ & $\cdots$ & \\
\hline $\mathrm{J} 1615+0546$ & M9 & $7 \pm 7$ & $17 \pm 4$ & $6.8 \pm 0.9$ & $6.8 \pm 2.2$ & $\ldots$ & \\
\hline J1645-1319 & $\mathrm{L} 1.5$ & $32 \pm 13$ & $\cdots$ & $27.0 \pm 0.8$ & $27.0 \pm 2.2$ & $26.58 \pm 0.06,26.4 \pm 1.0$ & 1,7 \\
\hline J1705-0516 & L0.5 & $10 \pm 7$ & $\ldots$ & $11.2 \pm 2.1$ & $11.2 \pm 2.9$ & $12.19 \pm 0.11,12.2 \pm 1.1$ & 1,7 \\
\hline J1707-0558AB & L0 & $2 \pm 2$ & $11 \pm 5$ & $2.9 \pm 0.9$ & $2.9 \pm 2.2$ & $\cdots$ & \\
\hline J1733-1654 & L0.5 & $14 \pm 2$ & $\ldots$ & $17.3 \pm 1.2$ & $17.3 \pm 2.3$ & $\ldots$ & \\
\hline J1745-1640 & L1.5 & $28 \pm 9$ & $\cdots$ & $26.2 \pm 1.1$ & $26.2 \pm 2.3$ & $\cdots$ & \\
\hline J1750-0016 & L5.5 & $19 \pm 3$ & $\ldots$ & $10 \pm 14$ & $19.3 \pm 3.4$ & $\ldots$ & \\
\hline J1845-6357 & M8.5 & $-18 \pm 6$ & $-9 \pm 3$ & $-18.2 \pm 0.5$ & $-18.2 \pm 2.1$ & $\ldots$ & \\
\hline $\mathrm{J} 2000-7523$ & M9 & $-3 \pm 22$ & $\ldots$ & $8.0 \pm 1.4$ & $8.0 \pm 2.4$ & $11.8 \pm 1.0$ & 3 \\
\hline $\mathrm{J} 2036+1051$ & L3 & $18 \pm 10$ & $\ldots$ & $21.5 \pm 3.2$ & $21.5 \pm 3.8$ & $19.7 \pm 0.5$ & 1 \\
\hline $\mathrm{J} 2037-1137$ & M8 & $-36 \pm 6$ & $-33 \pm 2$ & $-38.3 \pm 0.9$ & $-38.3 \pm 2.2$ & $-39 \pm 3$ & 5 \\
\hline $\mathrm{J} 2045-6332$ & M9 & $3 \pm 3$ & $12 \pm 11$ & $5.4 \pm 0.9$ & $5.4 \pm 2.2$ & $0.5 \pm 0.5$ & 3 \\
\hline $\mathrm{J} 2104-1037$ & L3 & $-25 \pm 2$ & $\ldots$ & $-21.2 \pm 1.0$ & $-21.2 \pm 2.2$ & $-21.09 \pm 0.12,-21 \pm 2$ & 1,7 \\
\hline $\mathrm{J} 2120+1021$ & M8 & $-43 \pm 9$ & $-37 \pm 6$ & $-42.8 \pm 0.9$ & $-42.8 \pm 2.2$ & $-31 \pm 5$ & 9 \\
\hline $\mathrm{J} 2200-3038 \mathrm{AB}$ & M8 & $-23 \pm 8$ & $\cdots$ & $-24.3 \pm 1.1$ & $-24.3 \pm 2.3$ & $-25 \pm 0.14,-25.3 \pm 1.0$ & 3,7 \\
\hline $\mathrm{J} 2226-7503$ & M8 & $18 \pm 10$ & $27 \pm 2$ & $16.5 \pm 0.9$ & $16.5 \pm 2.2$ & $15 \pm 3$ & 5 \\
\hline $\mathrm{J} 2306-0502$ & M8 & $-55 \pm 5$ & $-49 \pm 2$ & $-53.8 \pm 0.9$ & $-53.8 \pm 2.2$ & $-56 \pm 3,-52.80 \pm 0.16$ & 5,10 \\
\hline J2331-2749 & M7.5 & $-4 \pm 4$ & $0 \pm 8$ & $-4.1 \pm 1.1$ & $-4.1 \pm 2.3$ & $-4 \pm 3$ & 5 \\
\hline $\mathrm{J} 2337-0838$ & M8 & $-10 \pm 7$ & $-6 \pm 4$ & $-11.5 \pm 1.2$ & $-11.5 \pm 2.3$ & $\cdots$ & \\
\hline $\mathrm{J} 2346+1129$ & M8 & $-1 \pm 3$ & $9 \pm 6$ & $0.0 \pm 1.1$ & $0.0 \pm 2.3$ & $\ldots$ & \\
\hline $\mathrm{J} 2349+1224$ & M8 & $-3 \pm 5$ & $2 \pm 4$ & $-3.6 \pm 0.8$ & $-3.6 \pm 2.2$ & $-4 \pm 3,-2.00 \pm 0.10$ & 5,10 \\
\hline $\mathrm{J} 2351-2537$ & M8 & $-15 \pm 10$ & $-5 \pm 9$ & $-12.3 \pm 1.6$ & $-12.3 \pm 2.6$ & $-3.0 \pm 1.1,-10 \pm 3$ & 7,5 \\
\hline
\end{tabular}

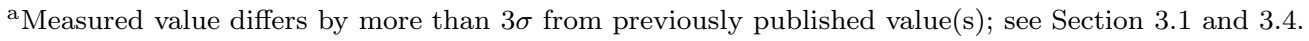

Note. - (1) Blake et al. (2010); (2) Bochanski et al. (2005); (3) Gálvez-Ortiz et al. (2010); (4) Mohanty \& Basri 
(2003); (5) Reiners \& Basri (2009); (6) Schmidt et al. (2010); (7) Seifahrt et al. (2010); (8) Tinnev \& Reid (1998); (9) West et al. (2008); (10) Tanner et al. (2012) 
Table 7. Final Radial Velocities and Heliocentric Space Motions

\begin{tabular}{|c|c|c|c|c|c|c|c|}
\hline Source & $\mathrm{SpT}$ & $\begin{array}{c}\text { Adopted RV } \\
\left(\mathrm{km} \mathrm{s}^{-1}\right)\end{array}$ & $\begin{array}{c}\mathrm{U} \\
\left(\mathrm{km} \mathrm{s}^{-1}\right)\end{array}$ & $\begin{array}{c}\mathrm{V} \\
\left(\mathrm{km} \mathrm{s}^{-1}\right)\end{array}$ & $\begin{array}{c}\mathrm{W} \\
\left(\mathrm{km} \mathrm{s}^{-1}\right)\end{array}$ & $P(\mathrm{TD}) / P(\mathrm{D})$ & Population $^{\mathrm{a}}$ \\
\hline J0004-2058 & M9 & $-33 \pm 2$ & $-44 \pm 3$ & $-13.1 \pm 1.5$ & $30 \pm 2$ & 0.04 & $\mathrm{D}$ \\
\hline J0024-0158 & M9.5 & $4 \pm 3$ & $10.8 \pm 0.4$ & $21.7 \pm 1.2$ & $7 \pm 2$ & 0.01 & $\mathrm{D}$ \\
\hline J0041-5621AB & M8 & $5 \pm 2$ & $6.5 \pm 1.0$ & $1.5 \pm 1.3$ & $5 \pm 2$ & 0.01 & $\mathrm{D}$ \\
\hline J0102-3737 & M9 & $-5 \pm 2$ & $-67 \pm 3$ & $-22.0 \pm 1.3$ & $13 \pm 2$ & 0.03 & $\mathrm{D}$ \\
\hline J0109-0343 & M8 & $-7 \pm 2$ & $-0.9 \pm 0.7$ & $1.6 \pm 0.7$ & $15 \pm 2$ & 0.01 & $\mathrm{D}$ \\
\hline J0123-6921 & M9 & $1 \pm 2$ & $4.5 \pm 1.8$ & $-5 \pm 2$ & $5 \pm 2$ & 0.01 & $\mathrm{D}$ \\
\hline J0148-3024 & M7.5 & $12 \pm 2$ & $12.3 \pm 0.8$ & $18.3 \pm 1.2$ & $-6 \pm 2$ & 0.01 & $\mathrm{D}$ \\
\hline J0248-1651 & M8 & $3 \pm 2$ & $22.3 \pm 1.6$ & $-4.6 \pm 1.6$ & \pm 2 & 0.01 & $\mathrm{D}$ \\
\hline J0331-3042 & M9 & $19 \pm 2$ & $17.0 \pm 1.2$ & $-9.0 \pm 1.3$ & $-7.8 \pm 1.8$ & 0.01 & $\mathrm{D}$ \\
\hline J0339-3525 & M9 & $6 \pm 2$ & $-1.9 \pm 0.8$ & $7.8 \pm 1.2$ & $8 \pm 2$ & 0.01 & $\mathrm{D}$ \\
\hline J0351-0052 & M7.5 & $-11 \pm 2$ & $35.8 \pm 1.8$ & $-11.2 \pm 0.7$ & $0.2 \pm 1.5$ & 0.01 & $\mathrm{D}$ \\
\hline $\mathrm{J} 0500+0330$ & $\mathrm{~L} 4$ & $5 \pm 3$ & $16 \pm 2$ & $-6.7 \pm 0.8$ & $-4.9 \pm 1.1$ & 0.01 & $\mathrm{D}$ \\
\hline J0517-3349 & M8 & $-39 \pm 2$ & $44.6 \pm 1.8$ & $13 \pm 3$ & $49 \pm 2$ & 0.50 & $\mathrm{D} / \mathrm{TD}$ \\
\hline J0544-2433 & M9 & $21 \pm 2$ & $41 \pm 4$ & $-35 \pm 3$ & $-9 \pm 2$ & 0.02 & $\mathrm{D}$ \\
\hline J0615-0100 & $\mathrm{L} 1$ & $-21 \pm 2$ & $36 \pm 2$ & $6 \pm 2$ & $28 \pm 2$ & 0.03 & $\mathrm{D}$ \\
\hline J0641-4322 & L3 & $74 \pm 2$ & $-55 \pm 5$ & $-52 \pm 2$ & $14 \pm 4$ & 0.15 & $\mathrm{D} / \mathrm{TD}$ \\
\hline J0652-2534 & M9 & $12 \pm 2$ & $-4.6 \pm 1.2$ & $12 \pm 2$ & $-8.1 \pm 0.5$ & 0.01 & $\mathrm{D}$ \\
\hline J0707-4900 & M8.5 & $113 \pm 2$ & $-37.1 \pm 1.6$ & $-92 \pm 2$ & $-17.1 \pm 0.9$ & 14.98 & $\mathrm{TD}$ \\
\hline J0751-2530 & L1 & $32 \pm 2$ & $-44.8 \pm 1.2$ & $4 \pm 2$ & $-47.1 \pm 0.8$ & 0.30 & $\mathrm{D} / \mathrm{TD}$ \\
\hline J0812-2444 & $\mathrm{L} 2.5$ & $-3 \pm 2$ & $30.7 \pm 1.0$ & $7 \pm 2$ & $11.0 \pm 0.2$ & 0.01 & $\mathrm{D}$ \\
\hline J0823-4912AB & L3 & $13 \pm 2$ & $1.1 \pm 0.2$ & $2 \pm 2$ & $-6.3 \pm 0.3$ & 0.01 & $\mathrm{D}$ \\
\hline J0830+0947 & M7.5 & $41 \pm 2$ & $-28.9 \pm 1.7$ & $-35 \pm 2$ & $-21 \pm 4$ & 0.03 & $\mathrm{D}$ \\
\hline J0835-0819 & L6 & $40 \pm 3$ & $-31 \pm 2$ & $-9 \pm 2$ & $9.2 \pm 1.4$ & 0.01 & $\mathrm{D}$ \\
\hline J0847-1532 & $\mathrm{L} 2$ & - \pm 2 & $28.2 \pm 1.0$ & $3.5 \pm 1.8$ & $6.5 \pm 0.6$ & 0.01 & $\mathrm{D}$ \\
\hline J0853-0329 & M9 & $7 \pm 2$ & $-1.9 \pm 1.3$ & $3.1 \pm 1.6$ & $-9.5 \pm 1.0$ & 0.01 & $\mathrm{D}$ \\
\hline J0902+0033 & M7 & $41 \pm 2$ & $-36 \pm 3$ & $-20.2 \pm 1.5$ & $-8 \pm 4$ & 0.01 & $\mathrm{D}$ \\
\hline J0909-0658 & $\mathrm{L} 1$ & $29 \pm 2$ & $-18 \pm 2$ & $-7.5 \pm 1.8$ & $6.2 \pm 1.8$ & 0.01 & $\mathrm{D}$ \\
\hline J0921-2104 & L1 & $81 \pm 2$ & $37 \pm 4$ & $-87 \pm 3$ & $8 \pm 2$ & 4.90 & $\mathrm{D} / \mathrm{TD}$ \\
\hline J0923+2340 & $\mathrm{L} 1$ & $-32 \pm 2$ & $66 \pm 4$ & $-19 \pm 4$ & $0 \pm 3$ & 0.02 & $\mathrm{D}$ \\
\hline J0949+0806 & M8.5 & $16 \pm 2$ & $34.7 \pm 1.4$ & $-54 \pm 2$ & $-5.0 \pm 1.7$ & 0.08 & $\mathrm{D}$ \\
\hline J1003-0105 & M8 & $22 \pm 2$ & $-30 \pm 3$ & $-7 \pm 2$ & $-1 \pm 3$ & 0.01 & $\mathrm{D}$ \\
\hline J1004-3335 & $\mathrm{L} 4$ & $-8 \pm 2$ & $41 \pm 3$ & $17 \pm 2$ & $1.0 \pm 0.9$ & 0.01 & $\mathrm{D}$ \\
\hline J1045-0149 & $\mathrm{L} 2$ & $1 \pm 3$ & $-20 \pm 3$ & $-3 \pm 2$ & $-2 \pm 2$ & 0.01 & $\mathrm{D}$ \\
\hline J1048-3956 & M8 & $-11 \pm 2$ & $0.2 \pm 0.4$ & $13 \pm 2$ & $-21.9 \pm 0.8$ & 0.01 & $\mathrm{D}$ \\
\hline $\mathrm{J} 1054+1214$ & M7.5 & $-7 \pm 2$ & $25 \pm 2$ & $12 \pm 2$ & $3 \pm 2$ & 0.01 & $\mathrm{D}$ \\
\hline $\mathrm{J} 1055+0808$ & M9 & $5 \pm 2$ & $-10 \pm 2$ & $-5 \pm 2$ & $-3 \pm 2$ & 0.01 & $\mathrm{D}$ \\
\hline $\mathrm{J} 1119+0828$ & M7 & $-45 \pm 2$ & $78 \pm 7$ & $14 \pm 3$ & $-30 \pm 2$ & 0.17 & $\mathrm{D} / \mathrm{TD}$ \\
\hline J1121-1313AB & M8.5 & $34 \pm 2$ & $-15.2 \pm 0.9$ & $-26.5 \pm 1.6$ & $17.0 \pm 1.5$ & 0.01 & $\mathrm{D}$ \\
\hline $\mathrm{J} 1134+0022$ & M9 & $33 \pm 2$ & $56 \pm 4$ & $-15 \pm 2$ & $32 \pm 2$ & 0.07 & $\mathrm{D}$ \\
\hline J1139-3159 & M9 & $7 \pm 2$ & $0.9 \pm 1.6$ & $-2 \pm 2$ & $1.5 \pm 1.5$ & 0.01 & $\mathrm{D}$ \\
\hline $\mathrm{J} 1155-3727$ & L3 & $46 \pm 2$ & $41.6 \pm 1.0$ & $-36 \pm 2$ & $-4.5 \pm 1.7$ & 0.02 & $\mathrm{D}$ \\
\hline $\mathrm{J} 1221+0257$ & Lo & $-8 \pm 3$ & $2 \pm 3$ & $9 \pm 3$ & $-2 \pm 3$ & 0.01 & $\mathrm{D}$ \\
\hline $\mathrm{J} 1224-1238$ & M9 & $-2 \pm 2$ & $-1.1 \pm 1.3$ & $-6 \pm 2$ & $-6 \pm 2$ & 0.01 & $\mathrm{D}$ \\
\hline $\mathrm{J} 1252+0252$ & M7 & $21 \pm 2$ & $34 \pm 3$ & $-16 \pm 3$ & $14 \pm 3$ & 0.01 & $\mathrm{D}$ \\
\hline $\mathrm{J} 1309-2330$ & M8 & $15 \pm 2$ & $27.9 \pm 1.1$ & $-9.9 \pm 1.4$ & $-3.6 \pm 1.4$ & 0.01 & $\mathrm{D}$ \\
\hline
\end{tabular}


Table 7-Continued

\begin{tabular}{|c|c|c|c|c|c|c|c|}
\hline Source & $\mathrm{SpT}$ & $\begin{array}{c}\text { Adopted RV } \\
\left(\mathrm{km} \mathrm{s}^{-1}\right)\end{array}$ & $\begin{array}{c}\mathrm{U} \\
\left(\mathrm{km} \mathrm{s}^{-1}\right)\end{array}$ & $\begin{array}{c}\mathrm{V} \\
\left(\mathrm{km} \mathrm{s}^{-1}\right)\end{array}$ & $\begin{array}{c}\mathrm{W} \\
\left(\mathrm{km} \mathrm{s}^{-1}\right)\end{array}$ & $P(\mathrm{TD}) / P(\mathrm{D})$ & Population $^{\mathrm{a}}$ \\
\hline $\mathrm{J} 1315-2649 \mathrm{AB}$ & L5 & $-8 \pm 3$ & $-41 \pm 5$ & $-39 \pm 6$ & $-14 \pm 3$ & 0.03 & $\mathrm{D}$ \\
\hline $\mathrm{J} 1332-0441$ & M7.5 & $-12 \pm 2$ & $9.5 \pm 1.3$ & $20.6 \pm 1.2$ & $-3 \pm 2$ & 0.01 & $\mathrm{D}$ \\
\hline $\mathrm{J} 1411-2119$ & M9 & - \pm 3 & $7.9 \pm 1.8$ & $5.9 \pm 1.3$ & $4.5 \pm 1.6$ & 0.01 & $\mathrm{D}$ \\
\hline $\mathrm{J} 1422+2116$ & M7 & $-4 \pm 2$ & $16.6 \pm 1.2$ & $17.8 \pm 1.0$ & $0 \pm 2$ & 0.01 & $\mathrm{D}$ \\
\hline $\mathrm{J} 1440+1339$ & M7 & $-5 \pm 2$ & $18.9 \pm 1.1$ & $-21.5 \pm 0.9$ & \pm 2 & 0.01 & $\mathrm{D}$ \\
\hline J1456-2809 & M7 & \pm 2 & $6.4 \pm 1.7$ & $-14.5 \pm 1.0$ & $-5.6 \pm 1.1$ & 0.01 & $\mathrm{D}$ \\
\hline J1500-0039 & M6 & $-13 \pm 2$ & $-11 \pm 3$ & $5.3 \pm 1.5$ & $9 \pm 3$ & 0.01 & $\mathrm{D}$ \\
\hline $\mathrm{J} 1501+2250$ & M9 & $6 \pm 2$ & $14.9 \pm 1.0$ & $10.9 \pm 0.6$ & $13 \pm 2$ & 0.01 & $\mathrm{D}$ \\
\hline $\mathrm{J} 1504-2355$ & M7.5 & $-29 \pm 2$ & $-26 \pm 2$ & $0 \pm 2$ & $1.1 \pm 1.5$ & 0.01 & $\mathrm{D}$ \\
\hline $\mathrm{J} 1506+1321$ & L3 & $1 \pm 3$ & $-28 \pm 4$ & $-35 \pm 5$ & $44 \pm 4$ & 0.30 & $\mathrm{D} / \mathrm{TD}$ \\
\hline J1510-0241 & M9 & $-41 \pm 2$ & $-36 \pm 2$ & $-5.5 \pm 1.8$ & $-5 \pm 2$ & 0.01 & $\mathrm{D}$ \\
\hline $\mathrm{J} 1510-2818$ & M9 & $-13 \pm 2$ & $-6 \pm 2$ & $6.9 \pm 1.1$ & $4.2 \pm 1.0$ & 0.01 & $\mathrm{D}$ \\
\hline J1534-1418 & M8 & $-71 \pm 2$ & $-66 \pm 3$ & $-24 \pm 4$ & $-13 \pm 2$ & 0.03 & $\mathrm{D}$ \\
\hline $\mathrm{J} 1539-0520$ & $\mathrm{~L} 4$ & $27 \pm 4$ & $50 \pm 3$ & $49.0 \pm 0.8$ & \pm 3 & 0.12 & $\mathrm{D} / \mathrm{TD}$ \\
\hline $\mathrm{J} 1550+1455 \mathrm{AB}$ & L3.5 & $13 \pm 2$ & $45 \pm 4$ & $9 \pm 3$ & $-3 \pm 4$ & 0.01 & $\mathrm{D}$ \\
\hline $\mathrm{J} 1553+1400$ & M9 & $-53 \pm 2$ & $-53 \pm 3$ & $-41 \pm 3$ & $11 \pm 3$ & 0.05 & $\mathrm{D}$ \\
\hline J1555-0956 & $\mathrm{L} 1$ & $15 \pm 2$ & $65 \pm 2$ & $13.32 \pm 0.13$ & $-50.4 \pm 1.5$ & 1.14 & $\mathrm{D} / \mathrm{TD}$ \\
\hline $\mathrm{J} 1607-0442$ & M9 & $11 \pm 2$ & $33 \pm 2$ & $-10.1 \pm 0.6$ & $-2.3 \pm 1.3$ & 0.01 & $\mathrm{D}$ \\
\hline $\mathrm{J} 1615+0546$ & M9 & $7 \pm 2$ & $24.4 \pm 1.8$ & $16.6 \pm 0.7$ & $0.000 \pm 1.6$ & 0.01 & $\mathrm{D}$ \\
\hline J1645-1319 & $\mathrm{L} 1.5$ & $27 \pm 2$ & $44 \pm 2$ & $-29.9 \pm 0.4$ & $7.5 \pm 0.8$ & 0.02 & $\mathrm{D}$ \\
\hline J1705-0516 & L0.5 & $11 \pm 3$ & $27 \pm 3$ & $13.7 \pm 0.7$ & $-1.8 \pm 1.1$ & 0.01 & $\mathrm{D}$ \\
\hline J1707-0558AB & L0 & $3 \pm 2$ & $15 \pm 2$ & $17.7 \pm 0.8$ & $2.1 \pm 1.0$ & 0.01 & $\mathrm{D}$ \\
\hline J1733-1654 & L0.5 & $17 \pm 2$ & $30 \pm 2$ & $15.3 \pm 1.5$ & $0.5 \pm 1.6$ & 0.01 & $\mathrm{D}$ \\
\hline $\mathrm{J} 1745-1640$ & $\mathrm{~L} 1.5$ & $26 \pm 2$ & $39 \pm 2$ & $14.0 \pm 1.5$ & $-4.6 \pm 1.4$ & 0.01 & $\mathrm{D}$ \\
\hline $\mathrm{J} 1750-0016$ & $\mathrm{~L} 5.5$ & $19 \pm 3$ & $24 \pm 3$ & $17.8 \pm 1.5$ & $30.5 \pm 0.9$ & 0.04 & $\mathrm{D}$ \\
\hline $\mathrm{J} 1845-6357$ & M8.5 & $-18 \pm 2$ & $-8.0 \pm 1.6$ & $48.1 \pm 0.9$ & $-25.1 \pm 0.8$ & 0.15 & $\mathrm{D} / \mathrm{TD}$ \\
\hline J2000-7523 & M9 & $8 \pm 2$ & $8.5 \pm 1.7$ & $1.5 \pm 1.6$ & $0.1 \pm 1.2$ & 0.01 & $\mathrm{D}$ \\
\hline $\mathrm{J} 2036+1051$ & L3 & $22 \pm 4$ & $39 \pm 3$ & $18 \pm 3$ & $1.6 \pm 1.8$ & 0.01 & $\mathrm{D}$ \\
\hline J2037-1137 & M8 & $-38 \pm 2$ & $-6.6 \pm 1.8$ & $-33 \pm 3$ & $14.6 \pm 1.4$ & 0.02 & $\mathrm{D}$ \\
\hline $\mathrm{J} 2045-6332$ & M9 & $5 \pm 2$ & $1.1 \pm 1.6$ & $-10.4 \pm 1.1$ & $0.9 \pm 1.3$ & 0.01 & $\mathrm{D}$ \\
\hline $\mathrm{J} 2104-1037$ & L3 & $-21 \pm 2$ & $-28.5 \pm 1.7$ & $-19.8 \pm 1.4$ & $-30 \pm 2$ & 0.03 & $\mathrm{D}$ \\
\hline $\mathrm{J} 2120+1021$ & M8 & $-43 \pm 2$ & $-17 \pm 2$ & $-25 \pm 2$ & $12 \pm 2$ & 0.01 & $\mathrm{D}$ \\
\hline $\mathrm{J} 2200-3038 \mathrm{AB}$ & M8 & $-24 \pm 2$ & $-32 \pm 8$ & $-1 \pm 5$ & $1 \pm 6$ & 0.01 & $\mathrm{D}$ \\
\hline $\mathrm{J} 2226-7503$ & M8 & $17 \pm 2$ & $17 \pm 2$ & $3 \pm 2$ & $-6 \pm 2$ & 0.01 & $\mathrm{D}$ \\
\hline $\mathrm{J} 2306-0502$ & M8 & $-54 \pm 2$ & $-32.1 \pm 1.1$ & $-53.9 \pm 1.6$ & $21 \pm 2$ & 0.14 & $\mathrm{D} / \mathrm{TD}$ \\
\hline J2331-2749 & M7.5 & $-4 \pm 2$ & $-15.6 \pm 1.0$ & $57.4 \pm 1.4$ & $10 \pm 2$ & 0.17 & $\mathrm{D} / \mathrm{TD}$ \\
\hline $\mathrm{J} 2337-0838$ & M8 & $-12 \pm 2$ & $-7 \pm 2$ & $1.0 \pm 1.7$ & $13 \pm 2$ & 0.01 & $\mathrm{D}$ \\
\hline $\mathrm{J} 2346+1129$ & M8 & \pm 2 & $50 \pm 3$ & $23.7 \pm 1.8$ & $11.9 \pm 1.8$ & 0.03 & $\mathrm{D}$ \\
\hline $\mathrm{J} 2349+1224$ & M8 & $-4 \pm 2$ & $19 \pm 2$ & $-3 \pm 2$ & $-3 \pm 2$ & 0.01 & $\mathrm{D}$ \\
\hline $\mathrm{J} 2351-2537$ & M8 & $-12 \pm 3$ & $-27 \pm 4$ & $8.9 \pm 1.1$ & $12 \pm 3$ & 0.01 & $\mathrm{D}$ \\
\hline
\end{tabular}

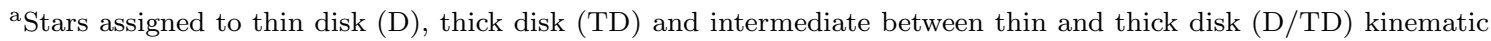
populations based on relative membership probabilities $P(\mathrm{TD}) / P(\mathrm{D})<0.1, P(\mathrm{TD}) / P(\mathrm{D})>10$ and $0.1<P(\mathrm{TD}) / P(\mathrm{D})$ $<10$, respectively; see Section 4.1 and Bensby et al. (2003). 
Table 8. Velocity Dispersions and Group Kinematic Ages

\begin{tabular}{|c|c|c|c|c|c|c|c|c|c|c|}
\hline Sample & $\mathrm{N}$ & $\begin{array}{c}\langle U\rangle \\
\left(\mathrm{km} \mathrm{s}^{-1}\right)\end{array}$ & $\begin{array}{c}\langle V\rangle \\
\left(\mathrm{km} \mathrm{s}^{-1}\right)\end{array}$ & $\begin{array}{c}\langle W\rangle \\
\left(\mathrm{km} \mathrm{s}^{-1}\right)\end{array}$ & $\begin{array}{c}\sigma_{U} \\
\left(\mathrm{~km} \mathrm{~s}^{-1}\right)\end{array}$ & $\begin{array}{c}\sigma_{V} \\
\left(\mathrm{~km} \mathrm{~s}^{-1}\right)\end{array}$ & $\begin{array}{c}\sigma_{W} \\
\left(\mathrm{~km} \mathrm{~s}^{-1}\right)\end{array}$ & $\begin{array}{c}\sigma_{v} \\
\left(\mathrm{~km} \mathrm{~s}^{-1}\right)\end{array}$ & $\begin{array}{l}\text { Age } \\
\text { (Gyr) }\end{array}$ & Note \\
\hline \multirow[t]{4}{*}{ All Sources } & 85 & $5.4 \pm 0.3$ & $-4.9 \pm 0.2$ & $1.4 \pm 0.3$ & $31.9 \pm 0.4$ & $25.0 \pm 0.3$ & $15.8 \pm 0.3$ & $43.5 \pm 0.3$ & $4.8 \pm 0.2$ & Unweighted \\
\hline & 85 & & & & $38.7 \pm 0.6$ & $27.1 \pm 0.4$ & $19.6 \pm 0.4$ & $51.1 \pm 0.6$ & $4.8 \pm 0.1$ & $|W|$ Weighted \\
\hline & 65 & & & & $34.4 \pm 0.4$ & $21.2 \pm 0.4$ & $10.7 \pm 0.4$ & $41.8 \pm 0.5$ & $4.3 \pm 0.2$ & Core \\
\hline & 36 & & & & $27 \pm 2$ & $34 \pm 8$ & $26 \pm 2$ & $52 \pm 6$ & $8 \pm 3$ & Wing \\
\hline \multirow{4}{*}{$d<20 \mathrm{pc}$} & 70 & $2.7 \pm 0.3$ & $-5.1 \pm 0.3$ & $1.1 \pm 0.3$ & $31.6 \pm 0.4$ & $27.0 \pm 0.3$ & $16.5 \pm 0.3$ & $44.8 \pm 0.4$ & $5.2 \pm 0.2$ & Unweighted \\
\hline & 70 & & & & $37.7 \pm 0.5$ & $28.6 \pm 0.4$ & $20.3 \pm 0.3$ & $51.5 \pm 0.4$ & $4.9 \pm 0.1$ & $|W|$ Weighted \\
\hline & 54 & & & & $35.0 \pm 0.5$ & $23.2 \pm 0.4$ & $11.3 \pm 0.4$ & $43.5 \pm 0.6$ & $4.8 \pm 0.3$ & Core \\
\hline & 30 & & & & $23 \pm 4$ & $35 \pm 7$ & $27 \pm 2$ & $51 \pm 5$ & $8 \pm 2$ & Wing \\
\hline \multirow[t]{4}{*}{ Late-M dwarfs } & 57 & $1.0 \pm 0.3$ & $-5.1 \pm 0.3$ & $2.1 \pm 0.3$ & $29.7 \pm 0.5$ & $24.0 \pm 0.2$ & $13.8 \pm 0.3$ & $40.6 \pm 0.4$ & $4.0 \pm 0.2$ & Unweighted \\
\hline & 57 & & & & $37.4 \pm 0.9$ & $28.5 \pm 0.4$ & $15.9 \pm 0.4$ & $49.5 \pm 0.6$ & $4.5 \pm 0.1$ & $|W|$ Weighted \\
\hline & 43 & & & & $28.7 \pm 0.6$ & $19.2 \pm 0.5$ & $11.3 \pm 0.4$ & $36.4 \pm 0.5$ & $2.9 \pm 0.1$ & Core \\
\hline & 24 & & & & $31 \pm 3$ & $36 \pm 7$ & $20 \pm 3$ & $52 \pm 5$ & $8 \pm 2$ & Wing \\
\hline \multirow[t]{2}{*}{ Not Young Late-M } & 48 & $0.8 \pm 0.4$ & $-6.5 \pm 0.3$ & $2.0 \pm 0.3$ & $32.3 \pm 0.5$ & $25.8 \pm 0.3$ & $15.0 \pm 0.3$ & $43.9 \pm 0.4$ & $5.0 \pm 0.2$ & Unweighted \\
\hline & 48 & & & & $38.5 \pm 0.9$ & $29.3 \pm 0.4$ & $16.5 \pm 0.4$ & $51.2 \pm 0.7$ & $4.8 \pm 0.1$ & $|W|$ Weighted \\
\hline \multirow[t]{2}{*}{ Normal Color Late-M } & 47 & $1.6 \pm 0.3$ & $-6.5 \pm 0.3$ & $1.3 \pm 0.3$ & $29.8 \pm 0.6$ & $25.1 \pm 0.3$ & $12.6 \pm 0.3$ & $40.9 \pm 0.5$ & $4.0 \pm 0.2$ & Unweighted \\
\hline & 47 & & & & $38.1 \pm 1.3$ & $30.5 \pm 0.4$ & $13.5 \pm 0.4$ & $50.6 \pm 1.2$ & $4.7 \pm 0.2$ & $|W|$ Weighted \\
\hline \multirow[t]{6}{*}{ L dwarfs } & 28 & $14.7 \pm 0.5$ & $-4.5 \pm 0.4$ & $0.000 \pm 0.4$ & $34.7 \pm 0.6$ & $27.5 \pm 0.6$ & $19.5 \pm 0.4$ & $48.4 \pm 0.5$ & $6.5 \pm 0.4$ & Unweighted \\
\hline & 28 & & & & $42.3 \pm 0.7$ & $24.7 \pm 0.9$ & $24.3 \pm 0.6$ & $54.8 \pm 0.8$ & $5.5 \pm 0.1$ & $|W|$ Weighted \\
\hline & 22 & & & & $37.8 \pm 0.9$ & $24.2 \pm 0.8$ & $10.1 \pm 0.7$ & $46.1 \pm 0.8$ & $5.7 \pm 0.4$ & Core \\
\hline & 12 & & & & $23 \pm 4$ & $35 \pm 1$ & $35 \pm 6$ & $57 \pm 7$ & $11 \pm 3$ & Wing \\
\hline & 11 & & & & $60 \pm 2$ & $38 \pm 2$ & $10.3 \pm 1.4$ & $72 \pm 2$ & $19.1 \pm 1.8$ & Core $\sigma<0$ \\
\hline & 11 & & & & $16 \pm 2$ & $11.9 \pm 1.5$ & $11.5 \pm 1.8$ & $23 \pm 2$ & $0.7 \pm 0.2$ & Core $\sigma>0$ \\
\hline \multirow[t]{2}{*}{ Not Young L } & 26 & $16.4 \pm 0.6$ & $-4.6 \pm 0.5$ & $0.0000 \pm 0.4$ & $35.4 \pm 0.7$ & $28.5 \pm 0.6$ & $20.2 \pm 0.4$ & $49.9 \pm 0.6$ & $7.1 \pm 0.4$ & Unweighted \\
\hline & 26 & & & & $43.4 \pm 0.8$ & $25.1 \pm 0.8$ & $24.8 \pm 0.5$ & $55.8 \pm 0.7$ & $5.7 \pm 0.1$ & $|W|$ Weighted \\
\hline \multirow[t]{2}{*}{ Normal Color Late-L } & 16 & $10.1 \pm 0.8$ & $-2.0 \pm 0.7$ & $3.1 \pm 0.5$ & $29.3 \pm 0.9$ & $18.9 \pm 0.9$ & $16.3 \pm 0.8$ & $38.5 \pm 1.0$ & $3.4 \pm 0.3$ & Unweighted \\
\hline & 16 & & & & $34.1 \pm 1.3$ & $23.0 \pm 1.6$ & $19.6 \pm 1.1$ & $45.6 \pm 1.3$ & $3.8 \pm 0.2$ & $|W|$ Weighted \\
\hline \multirow[t]{2}{*}{ Active L dwarfs } & 13 & $21.4 \pm 0.7$ & $-6.0 \pm 0.8$ & $-0.4 \pm 0.5$ & $34.4 \pm 1.1$ & $22.4 \pm 0.9$ & $20.4 \pm 0.8$ & $45.9 \pm 1.1$ & $5.6 \pm 0.4$ & Unweighted \\
\hline & 13 & & & & $43 \pm 2$ & $24.0 \pm 1.5$ & $27.0 \pm 1.1$ & $55.7 \pm 1.7$ & $5.6 \pm 0.3$ & $|W|$ Weighted \\
\hline \multirow[t]{2}{*}{ Inactive $\mathrm{L}$ dwarfs } & 15 & $8.7 \pm 0.7$ & $-3.1 \pm 0.5$ & $0.3 \pm 0.5$ & $35.2 \pm 0.9$ & $32.0 \pm 0.7$ & $19.5 \pm 0.5$ & $51.5 \pm 0.7$ & $7.8 \pm 0.5$ & Unweighted \\
\hline & 15 & & & & $39.1 \pm 0.8$ & $25.2 \pm 0.6$ & $22.1 \pm 0.4$ & $51.5 \pm 0.7$ & $4.9 \pm 0.1$ & $|W|$ Weighted \\
\hline \multirow[t]{2}{*}{ Unusually Red } & 9 & $-7.5 \pm 1.1$ & $2.6 \pm 0.7$ & $4.6 \pm 0.9$ & $27.9 \pm 1.4$ & $13.3 \pm 0.8$ & $8.8 \pm 0.9$ & $32.2 \pm 1.2$ & $2.0 \pm 0.2$ & Unweighted \\
\hline & 9 & & & & $28.4 \pm 1.3$ & $12.0 \pm 0.7$ & $6.3 \pm 0.6$ & $31.3 \pm 1.2$ & $1.6 \pm 0.2$ & $|W|$ Weighted \\
\hline \multirow[t]{2}{*}{ Unusually Blue } & 11 & $25.6 \pm 0.9$ & $-7.8 \pm 0.6$ & $-5.2 \pm 0.7$ & $40.4 \pm 1.2$ & $38.5 \pm 0.7$ & $24.0 \pm 0.5$ & $60.7 \pm 0.8$ & $12.4 \pm 0.9$ & Unweighted \\
\hline & 11 & & & & $49.5 \pm 1.0$ & $28.8 \pm 0.7$ & $26.4 \pm 0.5$ & $63.1 \pm 1.0$ & $7.0 \pm 0.2$ & $|W|$ Weighted \\
\hline
\end{tabular}


Table 8 - Continued

\begin{tabular}{|c|c|c|c|c|c|c|c|c|c|c|}
\hline Sample & $\mathrm{N}$ & $\begin{array}{c}\langle U\rangle \\
\left(\mathrm{km} \mathrm{s}^{-1}\right)\end{array}$ & $\begin{array}{c}\langle V\rangle \\
\left(\mathrm{km} \mathrm{s}^{-1}\right)\end{array}$ & $\begin{array}{c}\langle W\rangle \\
\left(\mathrm{km} \mathrm{s}^{-1}\right)\end{array}$ & $\begin{array}{c}\sigma_{U} \\
\left(\mathrm{~km} \mathrm{~s}^{-1}\right)\end{array}$ & $\begin{array}{c}\sigma_{V} \\
\left(\mathrm{~km} \mathrm{~s}^{-1}\right)\end{array}$ & $\begin{array}{c}\sigma_{W} \\
\left(\mathrm{~km} \mathrm{~s}^{-1}\right)\end{array}$ & $\begin{array}{c}\sigma_{v} \\
\left(\mathrm{~km} \mathrm{~s}^{-1}\right)\end{array}$ & $\begin{array}{l}\text { Age } \\
\text { (Gyr) }\end{array}$ & Note \\
\hline \multirow[t]{2}{*}{ Lithium } & 9 & $1.1 \pm 0.5$ & $2.0 \pm 0.5$ & $1.7 \pm 0.5$ & $4.8 \pm 0.5$ & $7.2 \pm 0.5$ & $5.6 \pm 0.4$ & $10.4 \pm 0.5$ & $<0.12$ & Unweighted \\
\hline & 9 & & & & $4.8 \pm 0.5$ & $6.3 \pm 0.6$ & $4.5 \pm 0.4$ & $9.0 \pm 0.5$ & $<0.04$ & $|W|$ Weighted \\
\hline \multirow[t]{2}{*}{ Low Surface Gravity } & 11 & $0.10 \pm 0.5$ & $1.2 \pm 0.5$ & $1.9 \pm 0.4$ & $7.8 \pm 0.7$ & $7.2 \pm 0.5$ & $5.3 \pm 0.3$ & $11.9 \pm 0.6$ & $<0.12$ & Unweighted \\
\hline & 11 & & & & $7.8 \pm 0.7$ & $7.2 \pm 0.6$ & $4.3 \pm 0.3$ & $11.6 \pm 0.6$ & $<0.05$ & $|W|$ Weighted \\
\hline
\end{tabular}

Note. - Ages for Unweighted, Core and Warm populations are based on Equation 5 using the full set of parameters given in Aumer \& Binney (2009). Ages for the $|W|$-Weighted populations are based on Equation 4 using parameters $\sigma_{v, 0}=10 \mathrm{~km} \mathrm{~s}^{-1}, \gamma_{v, p}=1.1 \times 10^{4}\left(\mathrm{~km} \mathrm{~s}^{-1}\right)^{3} \mathrm{Gyr}^{-1}$ and $T_{\gamma}=5$ Gyr Wielen 1977). 
Table 9. Average Ages of Simulated Populations

\begin{tabular}{|c|c|c|c|c|c|c|c|c|c|c|c|c|c|c|c|c|c|c|c|c|c|c|c|c|c|}
\hline \multirow{2}{*}{\multicolumn{2}{|c|}{$\frac{\beta}{\text { Dynamic Selection }^{\mathrm{b}}}$}} & \multicolumn{4}{|c|}{-0.5} & \multicolumn{4}{|c|}{0.0} & \multicolumn{4}{|c|}{0.5} & \multicolumn{4}{|c|}{1.0} & \multicolumn{4}{|c|}{$0.0 / 0.5^{\mathrm{a}}$} & \multicolumn{4}{|c|}{$0.5 / 0.0^{\mathrm{a}}$} \\
\hline & & \multicolumn{2}{|c|}{ No } & \multicolumn{2}{|c|}{ Yes } & \multicolumn{2}{|c|}{ No } & \multicolumn{2}{|c|}{ Yes } & \multicolumn{2}{|c|}{ No } & \multicolumn{2}{|c|}{ Yes } & \multicolumn{2}{|c|}{ No } & \multicolumn{2}{|c|}{ Yes } & \multicolumn{2}{|c|}{ No } & \multicolumn{2}{|c|}{ Yes } & \multicolumn{2}{|c|}{ No } & \multicolumn{2}{|c|}{ Yes } \\
\hline$\alpha$ & $\mathrm{SpT}$ & $\bar{\tau}$ & $\overline{\sigma_{\tau}}$ & $\overline{\bar{\tau}}$ & $\overline{\sigma_{\tau}}$ & $\bar{\tau}$ & $\overline{\sigma_{\tau}}$ & $\overline{\bar{\tau}}$ & $\overline{\sigma_{\tau}}$ & $\overline{\bar{\tau}}$ & $\overline{\sigma_{\tau}}$ & $\overline{\bar{\tau}}$ & $\overline{\sigma_{\tau}}$ & $\bar{\tau}$ & $\overline{\sigma_{\tau}}$ & $\overline{\bar{\tau}}$ & $\overline{\sigma_{\tau}}$ & $\bar{\tau}$ & $\overline{\sigma_{\tau}}$ & $\overline{\bar{\tau}}$ & $\overline{\sigma_{\tau}}$ & $\overline{\bar{\tau}}$ & $\overline{\sigma_{\tau}}$ & $\overline{\bar{\tau}}$ & $\overline{\sigma_{\tau}}$ \\
\hline \multirow[t]{2}{*}{-0.5} & M6-M9.5 & 1.5 & 1.4 & 1.4 & 1.3 & 3.5 & 2.3 & 3.0 & 2.3 & 6.0 & 1.8 & 5.5 & 2.1 & 6.9 & 0.9 & 6.9 & 1.0 & 5.8 & 2.1 & 5.5 & 2.3 & 3.6 & 2.3 & 3.3 & 2.3 \\
\hline & L0-L5.5 & 1.5 & 1.3 & 1.4 & 1.2 & 3.2 & 2.2 & 2.8 & 2.2 & 5.8 & 1.9 & 5.7 & 1.8 & 6.9 & 1.0 & 6.8 & 1.0 & 4.5 & 2.7 & 4.1 & 2.8 & 4.1 & 2.0 & 4.0 & 2.0 \\
\hline \multirow[t]{2}{*}{0.0} & M6-M9.5 & 1.5 & 1.4 & 1.2 & 1.1 & 3.4 & 2.3 & 2.9 & 2.2 & 6.0 & 1.7 & 5.7 & 1.9 & 6.9 & 1.0 & 6.9 & 0.9 & 5.6 & 2.1 & 5.5 & 2.2 & 3.6 & 2.3 & 3.2 & 2.1 \\
\hline & L0-L5.5 & 1.4 & 1.3 & 1.3 & 1.2 & 3.1 & 2.2 & 2.6 & 2.1 & 5.7 & 1.9 & 5.5 & 1.9 & 6.8 & 1.1 & 6.8 & 1.0 & 4.4 & 2.7 & 3.9 & 2.8 & 4.0 & 2.0 & 3.6 & 2.0 \\
\hline \multirow[t]{2}{*}{0.5} & M6-M9.5 & 1.5 & 1.4 & 1.4 & 1.3 & 3.4 & 2.3 & 2.8 & 2.2 & 6.0 & 1.8 & 5.7 & 2.1 & 6.9 & 1.0 & 6.9 & 1.1 & 5.6 & 2.2 & 5.2 & 2.4 & 3.5 & 2.3 & 2.9 & 2.2 \\
\hline & L0-L5.5 & 1.4 & 1.2 & 1.2 & 1.1 & 3.1 & 2.3 & 2.6 & 2.1 & 5.7 & 1.9 & 5.4 & 2.1 & 6.9 & 1.1 & 6.8 & 1.2 & 4.1 & 2.8 & 3.7 & 2.7 & 4.0 & 2.0 & 3.4 & 2.0 \\
\hline \multirow[t]{2}{*}{1.0} & M6-M9.5 & 1.4 & 1.4 & 1.4 & 1.3 & 3.4 & 2.3 & 3.0 & 2.2 & 5.9 & 1.8 & 5.7 & 2.0 & 6.9 & 1.0 & 6.9 & 1.2 & 5.5 & 2.2 & 5.2 & 2.5 & 3.5 & 2.3 & 2.9 & 2.2 \\
\hline & L0-L5.5 & 1.3 & 1.2 & 1.1 & 1.1 & 2.8 & 2.2 & 2.3 & 2.0 & 5.6 & 2.0 & 5.2 & 2.3 & 6.8 & 1.1 & 6.9 & 1.1 & 3.9 & 2.8 & 3.1 & 2.7 & 3.9 & 2.1 & 3.6 & 1.9 \\
\hline
\end{tabular}

${ }^{a}$ Different values of $\beta$ used for brown dwarfs (first number) and stars (second number).

${ }^{\mathrm{b}}$ Restricted to those sources whose orbits pass within 50 pc of the Sun after given initial Galactic radius and velocity components based on age-dependent dispersion functions; see Section 5. 
Table 10. Candidate Members of Young Moving Groups and Associations Based on BANYAN II Analysis

\begin{tabular}{|c|c|c|c|c|c|c|c|c|c|c|}
\hline Source & $\mathrm{SpT}$ & $\begin{array}{l}\text { Moving } \\
\text { Group }\end{array}$ & $P_{M}$ & $P_{C}$ & $\begin{array}{l}\Delta D \\
(\mathrm{pc})\end{array}$ & $\begin{array}{c}\Delta V \\
\left(\mathrm{~km} \mathrm{~s}^{-1}\right)\end{array}$ & $\mathrm{H} \alpha$ & $\mathrm{Li} \mathrm{I}$ & Low $g$ & Ref. \\
\hline J0041-5621AB & M6.5+M9 & THA & 100 & $<0.1$ & 7 & 1.4 & $\mathrm{Y}$ & $\mathrm{Y}$ & $\mathrm{N}$ & 1,2 \\
\hline J0123-6921 & M7.5 & THA & 99.9 & $<0.1$ & 11 & 2 & $\mathrm{Y}$ & Y & $\mathrm{Y}$ & 2 \\
\hline J0339-3525 & M9 & ARG & 17 & 0.3 & 25 & 13 & $\mathrm{Y}$ & $(\mathrm{Y})^{\mathrm{a}}$ & $\mathrm{Y}$ & 2 \\
\hline J0823-4912 & $\mathrm{L} 1.5$ & $\beta \mathrm{PMG}$ & 30 & 0.4 & 23 & 7 & $\mathrm{~N}$ & $\mathrm{Y}$ & $\mathrm{Y}$ & 3 \\
\hline J1139-3159 & M9 $\gamma$ & TWA & 99.6 & 0.1 & 21 & 5 & $\mathrm{Y}$ & $\mathrm{Y}$ & $\mathrm{Y}$ & 4 \\
\hline $\mathrm{J} 1510-2818$ & M9 & $\mathrm{ARG}$ & 60 & 34 & 24 & 4 & $\mathrm{Y}$ & $\mathrm{N}$ & $\mathrm{Y}$ & 5 \\
\hline $\mathrm{J} 2000-7523$ & M9 & $\beta \mathrm{PMG}$ & 99 & 4 & 20 & 2 & $\mathrm{Y}$ & $\mathrm{N}$ & $\mathrm{Y}$ & 1,2 \\
\hline J2045-6332 & M9 & $\beta \mathrm{PMG}$ & 87 & 0.2 & 11 & 8 & $\mathrm{Y}$ & $\mathrm{Y}$ & $\mathrm{Y}$ & 1,2 \\
\hline
\end{tabular}

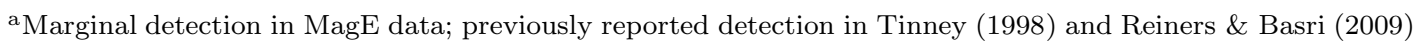

Note. - Moving groups and associations: $\mathrm{ABD}=\mathrm{AB}$ Doradus Moving Group, ARG $=$ Argus Association, $\beta \mathrm{PMG}=\beta$ Pictoris Moving Group, THA $=$ Tucana Horologium. $P_{M}$ gives the probability of membership, $P_{C}$ gives the probability of field contamination.

References. - (1) Gálvez-Ortiz et al. (2010); (2) Gizis (2002); (3) Sahlmann et al. (2015); (4) Gagné et al. (2014); (5) This paper.

Table 11. Polynomial Coefficients for Magnitude Difference Relations Between MKO and 2MASS Systems

\begin{tabular}{ccccc}
\hline \hline Filter & $c_{0}$ & $c_{1}$ & $c_{2}$ & $\sigma$ \\
& & & & \\
\hline$J$ & $-5.97605 \mathrm{e}-3$ & $-1.49741 \mathrm{e}-4$ & $-1.26157 \mathrm{e}-4$ & 0.0078 \\
$H$ & $-8.41293 \mathrm{e}-2$ & $9.70498 \mathrm{e}-3$ & $-1.58315 \mathrm{e}-4$ & 0.0052 \\
$K_{s}$ & $1.02933 \mathrm{e}-2$ & $-3.16442 \mathrm{e}-3$ & $7.96614 \mathrm{e}-5$ & 0.0053 \\
\hline
\end{tabular}

Note. - Coefficients are given for the polynomial relation MKO - 2MASS $=\sum_{i=0}^{2} c_{i}[S p T]^{i}$ where $\operatorname{SpT}(\mathrm{M} 0)=10, \operatorname{SpT}(\mathrm{M} 5)$ $=15, \operatorname{SpT}(\mathrm{L} 0)=20$, etc. 


\section{REFERENCES}

Allen, P. R., Koerner, D. W., Reid, I. N., \& Trilling, D. E. 2005, ApJ, 625, 385

Allers, K. N., \& Liu, M. C. 2013, ApJ, 772, 79

Andrei, A. H., Smart, R. L., Penna, J. L., et al. 2011, AJ, 141, 54

Antoja, T., Helmi, A., Dehnen, W., et al. 2014, A\&A, 563, A60

Antoja, T., Monari, G., Helmi, A., et al. 2015, ApJ, 800, L32

Aumer, M., \& Binney, J. J. 2009, MNRAS, 397, 1286

Baraffe, I., Chabrier, G., Barman, T. S., Allard, F., \& Hauschildt, P. H. 2003, A\&A, 402, 701

Bardalez Gagliuffi, D. C., Burgasser, A. J., Gelino, C. R., et al. 2014, ApJ, 794, 143

Barenfeld, S. A., Bubar, E. J., Mamajek, E. E., \& Young, P. A. 2013, ApJ, 766, 6

Barrado y Navascues, D. 1998, A\&A, 339, 831

Bartlett, J. L. 2007, PhD thesis, University of Virginia

Basri, G., \& Marcy, G. W. 1995, AJ, 109, 762

Basri, G., \& Reiners, A. 2006, AJ, 132, 663

Bastian, N., Covey, K. R., \& Meyer, M. R. 2010, ARA\&A, 48, 339

Benjamin, R. A. 2008, in Astronomical Society of the Pacific Conference Series, Vol. 387, Massive Star Formation: Observations Confront Theory, ed. H. Beuther, H. Linz, \& T. Henning, 375

Bensby, T., Feltzing, S., \& Lundström, I. 2003, A\&A, 410, 527

Berger, E. 2002, ApJ, 572, 503

Berger, E., Basri, G., Fleming, T. A., et al. 2010, ApJ, 709, 332

Bildsten, L., Brown, E. F., Matzner, C. D., \& Ushomirsky, G. 1997, ApJ, 482, 442

Biller, B. A., Kasper, M., Close, L. M., Brandner, W., \& Kellner, S. 2006, ApJ, 641, L141

Binks, A. S., \& Jeffries, R. D. 2014, MNRAS, 438, L11

Binney, J., Dehnen, W., \& Bertelli, G. 2000, MNRAS, 318, 658

Binney, J., \& Tremaine, S. 2008, Galactic Dynamics: Second Edition (Princeton University Press)

Binney, J., Burnett, B., Kordopatis, G., et al. 2014, MNRAS, 439, 1231 
Blake, C. H., Charbonneau, D., \& White, R. J. 2010, ApJ, 723, 684

Blake, C. H., Charbonneau, D., White, R. J., Marley, M. S., \& Saumon, D. 2007, ApJ, 666, 1198

Bochanski, J. J., Burgasser, A. J., Simcoe, R. A., \& West, A. A. 2011, AJ, 142, 169

Bochanski, J. J., Hawley, S. L., Covey, K. R., et al. 2010, AJ, 139, 2679

Bochanski, J. J., Hawley, S. L., Reid, I. N., et al. 2005, AJ, 130, 1871

Bochanski, J. J., Munn, J. A., Hawley, S. L., et al. 2007a, AJ, 134, 2418

Bochanski, J. J., West, A. A., Hawley, S. L., \& Covey, K. R. 2007b, AJ, 133, 531

Bochanski, J. J., Hennawi, J. F., Simcoe, R. A., et al. 2009, PASP, 121, 1409

Burgasser, A. J. 2004, ApJS, 155, 191

—. 2007, ApJ, 659, 655

- 2014, Proceedings of the 2013 International Workshop on Spectral Stellar Libraries, arXiv:1406.4887

Burgasser, A. J., Looper, D. L., Kirkpatrick, J. D., Cruz, K. L., \& Swift, B. J. 2008, ApJ, 674, 451

Burgasser, A. J., Melis, C., Zauderer, B. A., \& Berger, E. 2013, ApJ, 762, L3

Burgasser, A. J., Sitarski, B. N., Gelino, C. R., Logsdon, S. E., \& Perrin, M. D. 2011, ApJ, 739, 49

Burke, C. J., Pinsonneault, M. H., \& Sills, A. 2004, ApJ, 604, 272

Burrows, A., Hubbard, W. B., Lunine, J. I., \& Liebert, J. 2001, Reviews of Modern Physics, 73, 719

Caballero, J. A. 2007, ApJ, 667, 520

Carollo, D., Beers, T. C., Lee, Y. S., et al. 2008, Nature, 451, 216

Chabrier, G. 2003, PASP, 115, 763

Chen, B., Stoughton, C., Smith, J. A., et al. 2001, ApJ, 553, 184

Costa, E., Méndez, R. A., Jao, W.-C., et al. 2005, AJ, 130, 337

-. 2006, AJ, 132, 1234

Cruz, K. L., Kirkpatrick, J. D., \& Burgasser, A. J. 2009, AJ, 137, 3345

Cruz, K. L., Reid, I. N., Liebert, J., Kirkpatrick, J. D., \& Lowrance, P. J. 2003, AJ, 126, 2421 
Cruz, K. L., Reid, I. N., Kirkpatrick, J. D., et al. 2007, AJ, 133, 439

Cushing, M. C., Rayner, J. T., \& Vacca, W. D. 2005, ApJ, 623, 1115

Dahn, C. C., Harris, H. C., Vrba, F. J., et al. 2002, AJ, 124, 1170

Dauphole, B., \& Colin, J. 1995, A\&A, 300, 117

de la Reza, R., Torres, C. A. O., Quast, G., Castilho, B. V., \& Vieira, G. L. 1989, ApJ, 343, L61

De Simone, R., Wu, X., \& Tremaine, S. 2004, MNRAS, 350, 627

Deacon, N. R., \& Hambly, N. C. 2007, A\&A, 468, 163

Dehnen, W. 2000, AJ, 119, 800

Dehnen, W., \& Binney, J. J. 1998, MNRAS, 298, 387

Delfosse, X., Tinney, C. G., Forveille, T., et al. 1999, A\&AS, 135, 41

Delorme, P., Willott, C. J., Forveille, T., et al. 2008, A\&A, 484, 469

Dieterich, S. B., Henry, T. J., Jao, W.-C., et al. 2014, AJ, 147, 94

Douglas, S. T., Agüeros, M. A., Covey, K. R., et al. 2014, ApJ, 795, 161

Dupuy, T. J., Liu, M. C., \& Ireland, M. J. 2009a, ApJ, 692, 729

-. 2009b, ApJ, 699, 168

-. 2014, ApJ, 790, 133

Eisenbeiss, T., Ammler-von Eiff, M., Roell, T., et al. 2013, A\&A, 556, A53

Emerson, J. P., Sutherland, W. J., McPherson, A. M., et al. 2004, The Messenger, 117, 27

Epchtein, N., et al. 1997, The Messenger, 87, 27

Faherty, J. K., Burgasser, A. J., Cruz, K. L., et al. 2009, AJ, 137, 1

Faherty, J. K., Burgasser, A. J., Walter, F. M., et al. 2012, ApJ, 752, 56

Famaey, B., Pont, F., Luri, X., et al. 2007, A\&A, 461, 957

Faure, C., Siebert, A., \& Famaey, B. 2014, MNRAS, 440, 2564

Fleming, T. A., Schmitt, J. H. M. M., \& Giampapa, M. S. 1995, ApJ, 450, 401

Gagné, J., Lafrenière, D., Doyon, R., Malo, L., \& Artigau, É. 2014, ApJ, 783, 121

-. 2015, ApJ, 798, 73 
Gálvez-Ortiz, M. C., Clarke, J. R. A., Pinfield, D. J., et al. 2010, MNRAS, 409, 552

Gálvez-Ortiz, M. C., Kuznetsov, M., Clarke, J. R. A., et al. 2014, MNRAS, 439, 3890

Geballe, T. R., Knapp, G. R., Leggett, S. K., et al. 2002, ApJ, 564, 466

Gilmore, G., \& Reid, N. 1983, MNRAS, 202, 1025

Gizis, J. E. 2002, ApJ, 575, 484

Gizis, J. E., Monet, D. G., Reid, I. N., et al. 2000, AJ, 120, 1085

Hall, P. B. 2002a, ApJ, 580, L77

—. 2002b, ApJ, 564, L89

Hambly, N. C., Davenhall, A. C., Irwin, M. J., \& MacGillivray, H. T. 2001, MNRAS, 326, 1315

Hamuy, M., Suntzeff, N. B., Heathcote, S. R., et al. 1994, PASP, 106, 566

Hawley, S. L., Gizis, J. E., \& Reid, I. N. 1996, AJ, 112, 2799

Haywood, M., Di Matteo, P., Lehnert, M. D., Katz, D., \& Gómez, A. 2013, A\&A, 560, A109

Henry, T. J., Jao, W.-C., Subasavage, J. P., et al. 2006, AJ, 132, 2360

Henry, T. J., Subasavage, J. P., Brown, M. A., et al. 2004, AJ, 128, 2460

Ianna, P. A., \& Fredrick, L. W. 1995, ApJ, 441, L47

Jameson, R. F., Lodieu, N., Casewell, S. L., Bannister, N. P., \& Dobbie, P. D. 2008, MNRAS, 385, 1771

Johnson, D. R. H., \& Soderblom, D. R. 1987, AJ, 93, 864

Junqueira, T. C., Lépine, J. R. D., Braga, C. A. S., \& Barros, D. A. 2013, A\&A, 550, A91

Jurić, M., Ivezić, Ž., Brooks, A., et al. 2008, ApJ, 673, 864

Kaiser, N., Aussel, H., Burke, B. E., et al. 2002, in Society of Photo-Optical Instrumentation Engineers (SPIE) Conference Series, Vol. 4836, Society of Photo-Optical Instrumentation Engineers (SPIE) Conference Series, ed. J. A. Tyson \& S. Wolff, 154-164

Kalnajs, A. J. 1991, in Dynamics of Disc Galaxies, ed. B. Sundelius, 323

Kastner, J. H., Zuckerman, B., Weintraub, D. A., \& Forveille, T. 1997, Science, 277, 67

Kendall, T. R., Delfosse, X., Martín, E. L., \& Forveille, T. 2004, A\&A, 416, L17

Kendall, T. R., Jones, H. R. A., Pinfield, D. J., et al. 2007, MNRAS, 374, 445 
King, J. R., Villarreal, A. R., Soderblom, D. R., Gulliver, A. F., \& Adelman, S. J. 2003, AJ, 125, 1980

Kirkpatrick, J. D. 2005, ARA\&A, 43, 195

Kirkpatrick, J. D., Henry, T. J., \& McCarthy, Jr., D. W. 1991, ApJS, 77, 417

Kirkpatrick, J. D., Reid, I. N., Liebert, J., et al. 1999, ApJ, 519, 802

-. 2000, AJ, 120, 447

Kirkpatrick, J. D., Cruz, K. L., Barman, T. S., et al. 2008, ApJ, 689, 1295

Kirkpatrick, J. D., Looper, D. L., Burgasser, A. J., et al. 2010, ApJS, 190, 100

Kirkpatrick, J. D., Gelino, C. R., Cushing, M. C., et al. 2012, ApJ, 753, 156

Knapp, G. R., Leggett, S. K., Fan, X., et al. 2004, AJ, 127, 3553

Konopacky, Q. M., Ghez, A. M., Barman, T. S., et al. 2010, ApJ, 711, 1087

Kraus, A. L., Shkolnik, E. L., Allers, K. N., \& Liu, M. C. 2014, AJ, 147, 146

Kuzmin, G. 1956, AZh, 27

Lawrence, A., et al. 2007, MNRAS, 379, 1599

Lee, K.-G., Berger, E., \& Knapp, G. R. 2010, ApJ, 708, 1482

Lépine, S., Rich, R. M., \& Shara, M. M. 2007, ApJ, 669, 1235

Liu, M. C., Dupuy, T. J., \& Leggett, S. K. 2010, ApJ, 722, 311

Lodieu, N., Scholz, R.-D., McCaughrean, M. J., et al. 2005, A\&A, 440, 1061

Looper, D. L., Kirkpatrick, J. D., Cutri, R. M., et al. 2008, ApJ, 686, 528

López-Santiago, J., Montes, D., Crespo-Chacón, I., \& Fernández-Figueroa, M. J. 2006, ApJ, 643, 1160

Luhman, K. L. 2013, ApJ, 767, L1

Luhman, K. L., Stauffer, J. R., \& Mamajek, E. E. 2005, ApJ, 628, L69

Lutz, T. E., \& Upgren, A. R. 1980, AJ, 85, 1390

Luyten, W. J. 1980, NLTT Catalogue. Volume_III. 0__to -30_. (University of Minnesota)

Madau, P., Pozzetti, L., \& Dickinson, M. 1998, ApJ, 498, 106 
Magazzu, A., Martin, E. L., \& Rebolo, R. 1993, ApJ, 404, L17

Malo, L., Doyon, R., Feiden, G. A., et al. 2014, ApJ, 792, 37

Mamajek, E. E. 2012, ApJ, 754, L20

Mamajek, E. E., \& Bell, C. P. M. 2014, MNRAS, 445, 2169

Mamajek, E. E., Bartlett, J. L., Seifahrt, A., et al. 2013, AJ, 146, 154

Marshall, J. L., Burles, S., Thompson, I. B., et al. 2008, in Society of Photo-Optical Instrumentation Engineers (SPIE) Conference Series, Vol. 7014, Society of Photo-Optical Instrumentation Engineers (SPIE) Conference Series

Martin, E. L., Brandner, W., \& Basri, G. 1999, Science, 283, 1718

Martín, E. L., Delfosse, X., Basri, G., et al. 1999, AJ, 118, 2466

McElwain, M. W., \& Burgasser, A. J. 2006, AJ, 132, 2074

Minchev, I., \& Famaey, B. 2010, The Astrophysical Journal, 722, 112

Miyamoto, M., \& Nagai, R. 1975, PASJ, 27, 533

Mohanty, S., \& Basri, G. 2003, ApJ, 583, 451

Mohanty, S., Jayawardhana, R., \& Barrado y Navascués, D. 2003, ApJ, 593, L109

Monet, D. G., Dahn, C. C., Vrba, F. J., et al. 1992, AJ, 103, 638

Monnier, J. D., Che, X., Zhao, M., et al. 2012, ApJ, 761, L3

Murphy, S. J., \& Lawson, W. A. 2015, MNRAS, 447, 1267

Pavlenko, Y. V., Jones, H. R. A., Martín, E. L., et al. 2007, MNRAS, 380, 1285

Pérez Garrido, A., Lodieu, N., Béjar, V. J. S., et al. 2014, A\&A, 567, A6

Phan-Bao, N., Bessell, M. S., Martín, E. L., et al. 2008, MNRAS, 383, 831

Pineda, J. S., West, A. A., Bochanski, J. J., \& Burgasser, A. J. 2013, AJ, 146, 50

Pirzkal, N., Burgasser, A. J., Malhotra, S., et al. 2009, ApJ, 695, 1591

Quillen, A. C., Dougherty, J., Bagley, M. B., Minchev, I., \& Comparetta, J. 2011, MNRAS, 417, 762

Quillen, A. C., \& Minchev, I. 2005, AJ, 130, 576

Reid, I. N., Cruz, K. L., Kirkpatrick, J. D., et al. 2008, AJ, 136, 1290 
Reid, I. N., Gizis, J. E., \& Hawley, S. L. 2002a, AJ, 124, 2721

Reid, I. N., Hawley, S. L., \& Gizis, J. E. 1995, AJ, 110, 1838

Reid, I. N., Kirkpatrick, J. D., Gizis, J. E., \& Liebert, J. 1999, ApJ, 527, L105

Reid, I. N., Kirkpatrick, J. D., Liebert, J., et al. 2002b, AJ, 124, 519

Reid, M. J., Menten, K. M., Brunthaler, A., et al. 2014, ApJ, 783, 130

Reid, N., \& Majewski, S. R. 1993, ApJ, 409, 635

Reiners, A., \& Basri, G. 2006, AJ, 131, 1806

-. 2008, ApJ, 684, 1390

-. 2009, ApJ, 705, 1416

—. 2010, ApJ, 710, 924

Reiners, A., \& Christensen, U. R. 2010, A\&A, 522, A13+

Reiners, A., Seifahrt, A., \& Dreizler, S. 2010, A\&A, 513, L9

Ribas, I. 2003, A\&A, 400, 297

Roeser, S., Demleitner, M., \& Schilbach, E. 2010, AJ, 139, 2440

Ruiz, M. T., Takamiya, M. Y., \& Roth, M. 1991, ApJ, 367, L59

Sahlmann, J., Burgasser, A. J., Martín, E. L., et al. 2015, A\&A, submitted

Sahlmann, J., Lazorenko, P. F., Ségransan, D., et al. 2013, A\&A, 556, A133

Schmidt, S. J., Cruz, K. L., Bongiorno, B. J., Liebert, J., \& Reid, I. N. 2007, AJ, 133, 2258

Schmidt, S. J., West, A. A., Bochanski, J. J., Hawley, S. L., \& Kielty, C. 2014, PASP, 126, 642

Schmidt, S. J., West, A. A., Hawley, S. L., \& Pineda, J. S. 2010, AJ, 139, 1808

Scholz, R.-D. 2014, A\&A, 561, A113

Schönrich, R., Binney, J., \& Dehnen, W. 2010, MNRAS, 403, 1829

Seifahrt, A., Reiners, A., Almaghrbi, K. A. M., \& Basri, G. 2010, A\&A, 512, A37+

Sellwood, J. A., \& Binney, J. J. 2002, MNRAS, 336, 785

Sharma, S., Bland-Hawthorn, J., Binney, J., et al. 2014, ApJ, 793, 51

Siebert, A., Famaey, B., Minchev, I., et al. 2011, MNRAS, 412, 2026 
Simons, D. A., \& Tokunaga, A. 2002, PASP, 114, 169

Skrutskie, M. F., et al. 2006, AJ, 131, 1163

Skumanich, A. 1972, ApJ, 171, 565

Soderblom, D. R., Stauffer, J. R., MacGregor, K. B., \& Jones, B. F. 1993, ApJ, 409, 624

Spitzer, Jr., L., \& Schwarzschild, M. 1953, ApJ, 118, 106

Steinmetz, M., Zwitter, T., Siebert, A., et al. 2006, AJ, 132, 1645

Stephens, D. C., \& Leggett, S. K. 2004, PASP, 116, 9

Stephens, D. C., Leggett, S. K., Cushing, M. C., et al. 2009, ApJ, 702, 154

Strömberg, G. 1924, ApJ, 59, 228

Tanner, A., White, R., Bailey, J., et al. 2012, ApJS, 203, 10

Tinney, C. G. 1996, MNRAS, 281, 644

-. 1998, MNRAS, 296, L42+

Tinney, C. G., \& Reid, I. N. 1998, MNRAS, 301, 1031

Tinney, C. G., Reid, I. N., Gizis, J., \& Mould, J. R. 1995, AJ, 110, 3014

Tody, D. 1986, in Society of Photo-Optical Instrumentation Engineers (SPIE) Conference Series, Vol. 627, Society of Photo-Optical Instrumentation Engineers (SPIE) Conference Series, ed. D. L. Crawford, 733-+

Tokunaga, A. T., Simons, D. A., \& Vacca, W. D. 2002, PASP, 114, 180

Toomre, A. 1964, ApJ, 139, 1217

Torres, C. A. O., da Silva, L., Quast, G. R., de la Reza, R., \& Jilinski, E. 2000, AJ, 120, 1410

Torres, C. A. O., Quast, G. R., da Silva, L., et al. 2006, A\&A, 460, 695

Torres, C. A. O., Quast, G. R., Melo, C. H. F., \& Sterzik, M. F. 2008, Young Nearby Loose Associations, ed. Reipurth, B., 757

van Altena, W. F., Lee, J. T., \& Hoffleit, D. 1995, VizieR Online Data Catalog, 1174, 0

Walkowicz, L. M., Hawley, S. L., \& West, A. A. 2004, PASP, 116, 1105

Wegg, C., \& Gerhard, O. 2013, MNRAS, 435, 1874

Weinberger, A. J., Anglada-Escudé, G., \& Boss, A. P. 2013, ApJ, 762, 118 
West, A. A., \& Basri, G. 2009, ApJ, 693, 1283

West, A. A., Bochanski, J. J., Hawley, S. L., et al. 2006, AJ, 132, 2507

West, A. A., Hawley, S. L., Bochanski, J. J., et al. 2008, AJ, 135, 785

West, A. A., Hawley, S. L., Walkowicz, L. M., et al. 2004, AJ, 128, 426

West, A. A., Morgan, D. P., Bochanski, J. J., et al. 2011, AJ, 141, 97

Wielen, R. 1977, A\&A, 60, 263

Williams, M. E. K., Steinmetz, M., Binney, J., et al. 2013, MNRAS, 436, 101

Wilson, O. C. 1963, ApJ, 138, 832

Wright, E. L., Eisenhardt, P. R. M., Mainzer, A. K., et al. 2010, AJ, 140, 1868

Yoon, J., Peterson, D. M., Kurucz, R. L., \& Zagarello, R. J. 2010, ApJ, 708, 71

York, D. G., et al. 2000, AJ, 120, 1579

Zacharias, N., Finch, C., Girard, T., et al. 2010, AJ, 139, 2184

Zapatero Osorio, M. R., Martín, E. L., Béjar, V. J. S., et al. 2007, ApJ, 666, 1205

Zuckerman, B., Bessell, M. S., Song, I., \& Kim, S. 2006, ApJ, 649, L115

Zuckerman, B., \& Song, I. 2004, ARA\&A, 42, 685

Zuckerman, B., Song, I., \& Bessell, M. S. 2004, ApJ, 613, L65

Zuckerman, B., Song, I., Bessell, M. S., \& Webb, R. A. 2001, ApJ, 562, L87

Zuckerman, B., Vican, L., Song, I., \& Schneider, A. 2013, ApJ, 778, 5

Zuckerman, B., \& Webb, R. A. 2000, ApJ, 535, 959 